\title{
Third-Order Matching in the Presence of Type Constructors
}

\author{
Jan Springintveld* \\ Department of Philosophy \\ Utrecht University \\ P.O. Box 80126, 3508 TC Utrecht, The Netherlands
}

\begin{abstract}
We show that it is decidable whether a third-order matching problem in $\lambda \underline{\omega}$ (an extension of the simply typed lambda calculus with type constructors) has a solution or not. We present an algorithm which, given such a problem, returns a solution for this problem if the problem has a solution and returns fail otherwise. We also show that it is undecidable whether a third-order matching problem in $\lambda \underline{\omega}$ has a closed solution or not.
\end{abstract}

\section{Introduction}

It is well-known that type theory is a good basis for the implementation of proof checkers. Although there are various ways to use type theory for proof checking, they all exploit the fact that type theory provides a uniform way to represent and manipulate proofs, formulas and data types.

The man-machine interaction of proof checking can be considerably improved if some kind of matching algorithm can be implemented for the terms of the underlying type theory. For if one wants to prove $\phi(t)$ for a certain formula $\phi$ and term $t$, and one already has a proof $H$ of $\forall x \phi(x)$, one would like it to be sufficient to indicate that $H$ should be used without having to mention $t$. The proof checker should be able to match $\phi(x)$ with $\phi(t)$. Very often one is confronted with situations where $t$ is not just an object (natural number, boolean, ... ) but a function, or a functional, etc. To deal with this, one needs an algorithm for higher-order matching. So the question becomes: can we find such an algorithm? In other words: is the higher-order matching problem decidable for the underlying type theory? The starting point of this paper is the Calculus of Constructions $(\mathrm{CoC})$, a type theory which features polymorphism, dependent types and type constructors (see [3]). Unfortunately, while second-order matching is decidable in $\mathrm{CoC}$ ([4], [6], [7]), third-order matching is undecidable ([4], [5]). Since CoC forms the starting point for many type theoretical studies as well as for concrete implementations (e.g. [13], [15], [11], [16]), it is important to understand why third-order and hence higher-order matching is undecidable in $\mathrm{CoC}$ : is it a specific feature (polymorphism, dependent types, type constructors) that is responsible for the undecidability or is the undecidability caused by the interaction of these features? It is precisely for this

\footnotetext{
${ }^{*}$ This work is supported by the Netherlands Computer Science Research Foundation (SION) with financial support of the Netherlands Organisation for Scientific Research (NWO).
} 
kind of questions that Barendregt's $\lambda$-cube ([2]) was developed. This cube consists of eight systems, each extending the simply typed lambda calculus and each a subsystem of CoC. Starting from the simply typed lambda calculus the cube is erected by three systems, each supporting one of the abovementioned features: $\lambda P$ supports dependent types, $\lambda 2$ supports polymorphism and $\lambda \underline{\omega}$ supports type constructors. The other systems in the cube combine these features in all possible combinations. It is proved in [5] that in $\lambda P$ third-order matching is undecidable. In $\lambda 2$, higher-order matching is undecidable ([10]); it is (to our knowledge) an open question whether matching of finite order is decidable in $\lambda 2$. In $\lambda \omega$, the combination of $\lambda 2$ and $\lambda \underline{\omega}$, fourth-order matching is undecidable. It is (again to our knowledge) open whether third-order matching is decidable in $\lambda \omega$.

In this paper, we show that third-order matching is decidable in $\lambda \underline{\omega}$. In other words, we show that the mere presence of type constructors is not sufficient to make third-order matching undecidable. At first sight, this is not surprising, since $\lambda \underline{\omega}$ is a weak extension of the simply typed lambda calculus and in [8] it is proved that third-order matching is decidable in the simply typed lambda calculus (recently it is proved ([18], [19]) that fourth order matching is decidable as well). But it becomes more surprising when one realizes that the type structure of $\lambda \underline{\omega}$ is rich enough to encode the second-order unification problem in types of order 3. As is well-known, the second-order unification problem is undecidable (see [14]). And in fact, when one demands that solutions for matching problems are closed, in the sense that they do not contain new free variables, the third-order matching problem becomes undecidable in $\lambda \underline{\omega}$. It is not at all unnatural to demand this. For often one wants that the matching substitution is valid within the context one works and does not contain new variables that have to be instantiated further. The main argument in the proof of the decidability of general third-order matching is that when solutions are allowed to contain new, existential variables, second-order unification can be avoided by defining and solving matching problems in a suitable order.

This paper is organized as follows. In Section 2, we present the two typed lambda calculi with which we shall be concerned in this paper: $\lambda \tau$ (the simply typed lambda calculus with one base type $O$ ) and $\lambda \underline{\omega}$. In Section 3, we present terminology concerning matching problems and solutions. We also give an example which illustrates the difficulties that are involved in the proof of our main result. In Section 4, we show that third-order matching is decidable in $\lambda \underline{\omega}$ in case the terms are types. In Section 5 , we prove the decidability of third-order matching for objects in a special case; i.e. the case where there is a certain mild restriction on the order of every variable. In the appendix we show how to reduce the general case to the special case. In Section 6 we give a proof of the fact that it is undecidable whether a third-order matching problem in $\lambda \underline{\omega}$ has a closed solution or not. This proof is a variant of the undecidability proofs in [5].

Acknowledgements. I would like to thank Gilles Dowek for his hospitality during my stay at INRIA Rocquencourt and for the fruitful discussions we had there. I also thank Marc Bezem for carefully reading an earlier version of this paper and Jan Friso Groote, Jaco van de Pol and Alex Sellink for critical remarks and helpful suggestions. 


\section{The systems $\lambda \tau$ and $\lambda \underline{\omega}$}

In this section we introduce two typed lambda calculi: $\lambda \tau$, the simply typed lambda calculus with one base type $O$, and $\lambda \underline{\omega}$, the simply typed lambda calculus with type variables and type constructors (we consider this system with the Conversion Rule for $\beta \eta$-conversion). For more information on these systems the reader is referred to [2] or [13].

Definition 2.1 (Terms and reductions). Pseudo-terms are given by the following abstract syntax:

$$
\mathcal{T}::=\mathcal{C}|\mathcal{V}| \mathcal{T} \mathcal{T}|\lambda \mathcal{V}: \mathcal{T} . \mathcal{T}| \mathcal{T} \rightarrow \mathcal{T}
$$

Here $\mathcal{C}$ is an infinite set of constants and $\mathcal{V}$ is an infinite set of variables; $x, y, y^{\prime}, y_{1}, \ldots$ range over $\mathcal{V}$. Among the constants, three elements are singled out: $O, *$ and $\square$. Roman letters range over $\mathcal{T}$. We define $\mathcal{K}_{\square}::=* \mid \mathcal{K}_{\square} \rightarrow \mathcal{K}_{\square}$ and $\mathcal{K}_{*}::=O \mid \mathcal{K}_{*} \rightarrow \mathcal{K}_{*}$. We apply the usual conventions concerning brackets; so $A B C$ means $(A B) C$ and $A \rightarrow B \rightarrow C$ means $A \rightarrow(B \rightarrow C)$. The set of free variables of $A$ is defined as usual and denoted by $F V(A)$. Also the substitution of $A$ for $x$ in $B$ (denoted by $B[x:=A]$ ) and the relations $\rightarrow_{\beta}, \rightarrow_{\beta}, \rightarrow_{\eta}, \rightarrow_{\eta}, \rightarrow_{\beta \eta},=\beta$ and $=\beta \eta$ are defined on pseudo-terms as usual. Syntactic equality (modulo $\alpha$-conversion) is denoted by $\equiv$. The length of a term $A$ is the number of symbols that occur in $A$.

Definition 2.2 (Contexts and Judgements). In this paper $Q, Q_{1}, \ldots$ range over $\{\exists, \forall\}$. $Q x: B$ is called a (quantified) declaration. A (quantified) pseudo-context is a finite ordered sequence of quantified declarations $Q_{i} x_{i}: C_{i}$, where the $x_{i}$ are pairwise distinct. Pseudocontexts are denoted by capital Greek letters $\Delta, \Gamma, \Gamma_{0}, \ldots$ The empty context is denoted by \langle\rangle .

If $\exists x: C$ occurs in $\Gamma$, then $x$ is said to be existential in $\Gamma$. If $\forall x: C$ occurs in $\Gamma$, then $x$ is said to be universal in $\Gamma$. If every declaration in $\Gamma$ is of the form $\exists x: C$, then $\Gamma$ is an existential context.

The intuition behind the quantification of variables is that universal variables are considered to be constant, in the sense that solutions to matching problems are not allowed to substitute terms for them; substitutions are only allowed to substitute terms for existential variables.

If $\Gamma \equiv\left\langle Q_{1} x_{1}: A_{1}, \ldots, Q_{n} x_{n}: A_{n}\right\rangle$, then $F V(\Gamma):=\left\{x_{1}, \ldots, x_{n}\right\} \cup \bigcup_{1<i \leq n} F V\left(A_{i}\right)$, $\operatorname{dom}(\Gamma)=\left\{x_{1}, \ldots, x_{n}\right\}$ and $\Gamma, Q x: B$ denotes $\left\langle Q_{1} x_{1}: A_{1}, \ldots, Q_{n} x_{n}: A_{n}, \bar{Q} x: B\right\rangle$. (In general, we denote the concatenation of $\Gamma$ and $\Delta$ by $\Gamma, \Delta$.) Furthermore, for $1 \leq i \leq n, \Gamma_{x_{i}}$ denotes $\left\langle Q_{1} x_{1}: A_{1}, \ldots, Q_{i-1} x_{i-1}: A_{i-1}\right\rangle$ and $\Gamma^{x_{i}}$ denotes $\left\langle Q_{i+1} x_{i+1}: A_{1}, \ldots, Q_{n} x_{n}: A_{n}\right\rangle$. If the declaration $Q x: C$ occurs in $\Gamma$, then $\Gamma(x)$ denotes $C$. We write $\Gamma \subseteq \Delta$ if each $Q x: A$ in $\Gamma$ also occurs in $\Delta$; we call such $\Delta$ an extension of $\Gamma$.

A judgement is of the form $\Gamma \vdash A: B$, where $\Gamma$ is a quantified pseudo-context and $A$ and $B$ are pseudo-terms. When we want to indicate that a judgement is derived in a system $\lambda \circ$, we write $\Gamma \vdash_{\lambda_{0}} A: B$. If $\Gamma \vdash_{\lambda_{0}} A: B$, then we call $A$ closed in $\Gamma$ if all free variables $x$ in $A$ are universal in $\Gamma$ and moreover $\Gamma(x)$ is closed in $\Gamma_{x}$. It is easily seen that if $A$ is closed in $\Gamma$ and $\Delta$ is a legal extension of $\Gamma$ then $A$ is closed in $\Delta$.

A pseudo-term $A$ is called legal when there exist a pseudo-context $\Gamma$ and a pseudo-term $B$ such that $\Gamma \vdash A: B$ or $\Gamma \vdash B: A$. A pseudo-context $\Gamma$ is called legal when there exist pseudo-terms $A$ and $B$ such that $\Gamma \vdash A: B$. 


\begin{tabular}{|lll|}
\hline Axiom & \langle\rangle$\vdash c: s$ & if $c: s \in A x_{\lambda \circ}$ \\
Weart & $\frac{\Gamma \vdash B: s}{\Gamma, Q x: B \vdash x: B}$ & if $x \notin F V(\Gamma)$ \\
Product & $\frac{\Gamma \vdash A: B \quad \Gamma \vdash B^{\prime}: s}{\Gamma, Q x: B^{\prime} \vdash A: B}$ & if $x \notin F V(\Gamma)$ \\
Application & $\frac{\Gamma \vdash A_{1}: B_{1} \rightarrow B_{2} \quad \Gamma \vdash A_{2}: B_{1}}{\Gamma \vdash A_{1} A_{2}: B_{2}}$ & \\
Abstraction & $\frac{\Gamma, Q x: A_{1} \vdash A_{2}: B_{2} \quad \Gamma \vdash A_{1} \rightarrow B_{2}: s}{\Gamma \vdash \lambda x: A_{1} \cdot A_{2}: A_{1} \rightarrow B_{2}}$ & \\
& $\frac{\Gamma \vdash A: B^{\prime} \Gamma \vdash B: s}{\Gamma \vdash A: B}$ & \\
Conversion & $\frac{}{\Gamma}$ & \\
\hline
\end{tabular}

Table 1: The rules

Definition 2.3 (The systems). The systems $\lambda \tau$ and $\lambda \underline{\omega}$ are defined using the rules in Table 1. Here $\operatorname{Sort}_{\lambda \circ} \subseteq \mathcal{C}$ is a set of sorts and $s$ ranges over $\operatorname{Sort}_{\lambda \circ}$. Furthermore $A x_{\lambda \circ}$ is a set of statements of the from $c: s$, where $c \in \mathcal{C}$. The systems $\lambda \tau$ and $\lambda \underline{\omega}$ can be obtained from the rules by taking $\operatorname{Sort}_{\lambda \tau}=\{*\}$, $\operatorname{Sort}_{\lambda \underline{\omega}}=\{*, \square\}, A x_{\lambda \tau}=\{O: *\}$ and $A x_{\lambda \underline{\omega}}=\{*: \square\}$. In this paper we let $\lambda \circ$ range over $\lambda \tau$ and $\bar{\lambda} \underline{\omega}$ and (except in Table 1 ) $s$ over $\{*, \square\}$.

We fix some more notation.

Notation 2.4. Let $\Gamma$ be $\left\langle x_{1}: A_{1}, \ldots, x_{n}: A_{n}\right\rangle$ and let $\Delta$ be $\left\langle x_{1}: B_{1}, \ldots, x_{n}: B_{n}\right\rangle$. Then we write $\Gamma \rightarrow_{\beta} \Delta$ if $A_{i} \rightarrow_{\beta} B_{i}$, for some $i, 1 \leq i \leq n$, and for all $1 \leq j \leq n, j \neq i$, we have $A_{j} \equiv B_{j}$. Similarly for $\Gamma \rightarrow_{\eta} \Delta$. From this the notions $\Gamma \rightarrow_{\beta} \Delta, \Gamma \rightarrow_{\eta} \Delta, \Gamma \rightarrow_{\beta \eta} \Delta, \Gamma={ }_{\beta} \Delta$, $\Gamma={ }_{\beta \eta} \Delta$ can be defined as usual.

We write $\Gamma \subseteq_{\square} \Delta$ if for all $Q x: A$ in $\Gamma$ such that $A \in \mathcal{K}_{\square}$ also $Q x: A$ in $\Delta$. So every type that is well-typed in $\Gamma$ is also well-typed in $\Delta$ (provided $\Gamma$ and $\Delta$ are legal). From $\subseteq_{\square},=_{\square}$ is defined as usual. We use the abbreviation $\Gamma \vdash A: B: C$ for $\Gamma \vdash A: B$ and $\Gamma \vdash B: C$.

The following meta-theoretic properties hold in $\lambda \tau$ and $\lambda \underline{\omega}$. The results are taken from [1], [2], [13], and [12].

\section{Theorem 2.5.}

1. (Substitutivity) If $A \rightarrow_{\beta \eta} A^{\prime}$, then $A[x:=B] \rightarrow_{\beta \eta} A^{\prime}[x:=B]$. 
2. (Substitution Lemma) Suppose $\Gamma, Q x: C, \Delta \vdash_{\lambda_{\circ}} A: B$ and $\Gamma \vdash_{\lambda_{\circ}} D: C$. Then $\Gamma, \Delta[x:=D] \vdash_{\lambda_{0}} A[x:=D]: B[x:=D]$.

3. (Strong Normalization). If $\Gamma \vdash_{\lambda \circ} A: B$, then there are no infinite $\beta \eta$-reduction sequences starting from $A$ or from $B$.

4. (Confluence) If $\Gamma \vdash_{\lambda \circ} A_{1}: B$ and $\Gamma \vdash_{\lambda_{\circ}} A_{2}: B$ and $A_{1}={ }_{\beta \eta} A_{2}$, then there exists a term $A_{3}$ such that $A_{1} \rightarrow \beta \eta A_{3}$ and $A_{2} \rightarrow \beta \eta A_{3}$.

5. (Subject Reduction) If $\Gamma \vdash_{\lambda_{0}} A: B$ and $A \rightarrow_{\beta \eta} A^{\prime}$, then $\Gamma \vdash_{\lambda_{0}} A^{\prime}: B$. If $\Gamma \rightarrow_{\beta \eta} \Gamma^{\prime}$, then $\Gamma^{\prime} \vdash_{\lambda_{0}} A: B$.

6. (Unicity of Types) If $\Gamma \vdash_{\lambda_{0}} A: B$ and $\Gamma \vdash_{\lambda_{0}} A: B^{\prime}$, then $B={ }_{\beta \eta} B^{\prime}$.

7. (Thinning) If $\Gamma \vdash_{\lambda_{\circ}} A: B, \Gamma \subseteq \Delta$ and $\Delta$ is legal in $\lambda \circ$, then $\Delta \vdash_{\lambda_{\circ}} A: B$.

8. (Strengthening). If $\Gamma, x: C, \Delta \vdash_{\lambda \circ} A: B$ and $x \notin F V(\Delta) \cup F V(A) \cup F V(B)$, then $\Gamma, \Delta \vdash_{\lambda \circ} A: B$.

9. (Permutation) If $\Gamma, x: C, y: D, \Delta \vdash_{\lambda \circ} A: B$ and $\Gamma \vdash_{\lambda \circ} D: s$, then $\Gamma, y: D, x: C, \Delta \vdash_{\lambda \circ}$ $A: B$.

For $\lambda \tau$ we can replace $=\beta \eta$ in (5) by $\equiv$. This implies that in $\lambda \tau$ the Conversion Rule is redundant.

By Confluence and Strong Normalization we know that each legal term $A$ has a unique $\beta \eta$ normal form. We denote it by $\operatorname{nf}(A)$. A term $A$ is called normal when $\operatorname{nf}(A) \equiv A$. Next, we give some more basic facts concerning the terms in our systems. The proofs of these facts are standard and omitted.

\section{Lemma 2.6.}

1. $\Gamma \vdash_{\lambda \underline{\omega}} A: \square \Leftrightarrow\left(\Gamma\right.$ legal and $\left.A \in \mathcal{K}_{\square}\right)$. Such $A$ are called kinds. * is called an atomic kind; all other kinds are called arrow kinds.

2. $\Gamma \vdash_{\lambda \tau} A: s \Leftrightarrow\left(\Gamma\right.$ legal and $s \equiv *$ and $\left.A \in \mathcal{K}_{*}\right)$.

3. $\Gamma \vdash_{\lambda_{0}} A: s$, then for all $x \in \operatorname{dom}(\Gamma)$ such that $\Gamma_{x} \vdash_{\lambda_{0}} \Gamma(x): *$ we have: $x \notin F V(A)$. So A contains no object variables.

4. For no $A$ it is true that \langle\rangle$\vdash_{\lambda \underline{\omega}} A: *$. So there exist no closed types in $\lambda \underline{\omega}$.

5. If $\Gamma \vdash_{\lambda_{\circ}} A: *$ and $\Gamma, A$ are normal, then $A$ is called a type and $A$ is of one of the three following forms:

- $O$.

- $x A_{1} \ldots A_{n}$, for some $n \geq 0$, where (for $0 \leq i \leq n$ ) $A_{i}$ is normal and $\Gamma \vdash_{\lambda_{\circ}} A_{i}: B_{i}$ : $\square$, for some term $B_{i}$.

- $A_{1} \rightarrow A_{2}$, where (for $\left.i=1,2\right) A_{i}$ is normal and $\Gamma \vdash_{\lambda_{0}} A_{i}: *$. 
In the first two cases, $A$ is called an atomic type. In the third case, $A$ is called an arrow type.

6. If $\Gamma \vdash_{\lambda \circ} A: B: *$ and $\Gamma, A$ are normal, then $A$ is of one of the two following forms:

- $x A_{1} \ldots A_{n}$, for some $n \geq 0$, where (for $\left.1 \leq i \leq n\right) A_{i}$ is normal and $\Gamma \vdash_{\lambda_{\circ}} A_{i}: B_{i}: *$ for some term $B_{i}$.

- $\lambda x: A_{1} \cdot A_{2}$, where $A_{1}$ is as described in (5), $A_{2}$ is normal and $\Gamma, x: A_{1} \vdash_{\lambda_{0}} A_{2}$ : $B_{2}: *$, for some term $B_{2}$.

In the first case, $A$ is called an atomic term. In the second case, $A$ is called an abstraction term.

7. Suppose $\Gamma \vdash_{\lambda_{0}} A: s$, where $A$ is normal. Using the brackets convention we can write $A$ uniquely as $A_{1} \rightarrow \cdots \rightarrow A_{n} \rightarrow B$, with $n \geq 0$ and $B$ atomic. Unless stated otherwise, we assume that types and kinds are written in this way.

8. Suppose $\Gamma \vdash_{\lambda \circ} A: B$. There exist $\Gamma_{1}, \Gamma_{2} \subseteq \Gamma$ such that $\Gamma_{1}, \Gamma_{2} \vdash_{\lambda \circ} A: B$ and for all declarations $Q x: C$ in $\Gamma$ we have: $Q x: C \in \Gamma_{1} \Leftrightarrow C \in \mathcal{K}_{\square} . \Gamma_{1}$ and $\Gamma_{2}$ are unique up to the order of the declarations. The variables in $\Gamma_{1}$ are called constructor variables; the variables in $\Gamma_{2}$ are called object variables. From now on we assume that contexts are in this form.

It will be convenient to assume that terms are in $\eta$-long- $\beta$-normal form. This notion is defined below.

Definition 2.7. Suppose $\Gamma \vdash_{\lambda \circ} A: B$, where all terms are normal. We $\operatorname{define~} \operatorname{lnf}_{\Gamma}(A)$ by induction.

- $A \in \mathcal{C}$. Then $\operatorname{lnf}_{\Gamma}(A) \equiv A$.

- $A \equiv \lambda x: A_{1} \cdot A_{2}$. Then $\operatorname{lnf}_{\Gamma}(A) \equiv \lambda x: \operatorname{lnf}_{\Gamma}\left(A_{1}\right) \cdot \operatorname{lnf}_{\Gamma, \forall x: A_{1}}\left(A_{2}\right)$.

- $A \equiv A_{1} \rightarrow A_{2}$. Then $\operatorname{lnf}_{\Gamma}(A) \equiv \operatorname{lnf}_{\Gamma}\left(A_{1}\right) \rightarrow \operatorname{lnf}_{\Gamma}\left(A_{2}\right)$.

- $A \equiv x A_{1} \ldots A_{n}$. Write $B$ as $B_{1} \rightarrow \cdots \rightarrow B_{m} \rightarrow C$. Then

$$
\operatorname{lnf}_{\Gamma}(A) \equiv \lambda y_{1}: B_{1}^{\prime} \ldots \lambda y_{m}: B_{m}^{\prime} \cdot x A_{1}^{\prime} \ldots A_{n}^{\prime} y_{1}^{\prime} \ldots y_{m}^{\prime}
$$

where $B_{i}^{\prime} \equiv \operatorname{lnf}_{\Gamma}\left(B_{i}\right)($ for $1 \leq i \leq m), A_{i}^{\prime} \equiv \operatorname{lnf}_{\Gamma}\left(A_{i}\right)$ (for $1 \leq i \leq n$ ) and $y_{i}^{\prime} \equiv$ $\operatorname{lnf}_{\Gamma, \forall y_{i}: B_{i}}\left(y_{i}\right)($ for $1 \leq i \leq m)$.

(For the well-foundedness of this definition, see [7]). This definition is extended to non-normal terms as follows. Suppose $\Gamma \vdash_{\lambda \circ} A: B$. Then $\operatorname{lnf}_{\Gamma}(A):=\operatorname{lnf}_{\mathrm{nf}_{(\Gamma)}}(\operatorname{nf}(A))$. This definition is justified by Theorem 2.5. If $\Gamma \vdash_{\lambda \circ} A: B$, then $A$ is said to be in $L N F \operatorname{if}_{\lambda^{\prime}} \operatorname{lnf}_{\Gamma}(A) \equiv A$. Unless stated otherwise, we assume that terms are in LNF.

Lemma 2.6 remains true when we replace 'normal' by 'in LNF'. 
Lemma 2.8. Suppose $\Gamma \vdash_{\lambda \circ} A: A_{1} \rightarrow \cdots \rightarrow A_{n} \rightarrow B$, where all terms are in LNF. Then $A \equiv \lambda x_{1}: A_{1} \ldots \lambda x_{n}: A_{n} . x B_{1} \ldots B_{m}$, for some variable $x$ (possibly among $\left\{x_{1}, \ldots, x_{n}\right\}$ ) and terms $B_{1}, \ldots, B_{m}$ (in $L N F$ ).

We need a slightly non-standard notion of head normal form for types. First we say what a domain is. If $A$ has a subterm of the form $\lambda x: A_{1} \cdot A_{2}$, then $A_{1}$ is called a domain in $A$.

Definition 2.9. In this definition terms are not assumed to be in LNF.

1. Let $\Gamma \vdash_{\lambda \circ} A: s$. Then $A$ is in head-normal form $(H N F)$ if $A \equiv O, A \equiv *, A \equiv x A_{1} \ldots A_{n}$ or $A \equiv A_{1} \rightarrow A_{2}$ with $A_{1}, A_{2}$ in HNF.

2. A term $A$ is in $D-H N F$ if every domain in $A$ is in HNF.

Note that a term in LNF is also in (D)-HNF and that we can speak of (legal) contexts that are normal, in HNF or in LNF.

The following technical lemmas are used in Section 5. The first lemma states that if a term $A$ is closed after substitution of terms of atomic type for universal variables, then $A$ is itself closed. The second lemma states that if a term $A$ after substitution of a second-order term $\lambda y_{1}: S_{1} \ldots \lambda y_{n}: S_{n} . B^{\prime}$ reduces to a closed term $C$ then $B^{\prime}$ is itself closed.

Lemma 2.10. Suppose

1. $\Gamma, \forall x_{1}: S_{1}, \ldots, \forall x_{n}: S_{n}, \Delta \vdash_{\lambda \circ} A: T$, where $S_{i}$ is atomic, for each $1 \leq i \leq n$;

2. $\Gamma, \Delta \vdash_{\lambda_{0}} B: T$, where $B$ is closed in $\Gamma, \Delta$;

3. $\Gamma \vdash_{\lambda \circ} C_{i}: S_{i}(1 \leq i \leq n)$;

4. $A[\vec{x}:=\vec{C}] \rightarrow \beta \eta B$.

Then $A$ is closed in $\Gamma, \forall x_{1}: S_{1}, \ldots, \forall x_{n}: S_{n}, \Delta$.

Proof. By induction on the LNF-structure of $A$.

Lemma 2.11. Let $B$ be $\lambda y_{1}: S_{1} \ldots \lambda y_{n}: S_{n} . B^{\prime}$. Suppose

1. $\Gamma, \forall x: S_{1} \rightarrow \cdots \rightarrow S_{n} \rightarrow S, \Delta \vdash_{\lambda \circ} A: T$, where $n \geq 0, S_{i}$ (for $1 \leq i \leq n$ ) and $S$ are atomic and $x \in F V(A)$.

2. $\Gamma \vdash_{\lambda \circ} B: S_{1} \rightarrow \cdots \rightarrow S_{n} \rightarrow S: *$;

3. $\Gamma, \Delta \vdash_{\lambda_{0}} C: T$, where $C$ is closed in $\Gamma, \Delta$;

4. $A[x:=B] \rightarrow \beta \eta C$.

Then $B^{\prime}$ is closed in $\Gamma, \forall y_{1}: S_{1}, \ldots, \forall y_{n}: S_{n}$ and $S$ is closed in $\Gamma$. Moreover, every variable free in $B^{\prime}$ but not among $\left\{y_{1}, \ldots, y_{n}\right\}$ occurs in $C$ (hence in every legal $\beta \eta$-expansion of $C$ ).

Proof. By induction on the LNF-structure of $A$, using Lemma 2.10. 


\section{Matching problems}

This section recalls some definitions from [7], [9].

Definition 3.1. Suppose $\Gamma \vdash_{\lambda \circ} A: s$, where $A$ is in HNF. We define $\operatorname{ord}_{\Gamma}(A)$, the order of $A$ in $\Gamma$, as follows.

$\operatorname{ord}_{\Gamma}(A)= \begin{cases}2 & \text { if } A \equiv * \\ 1 & \text { if } A \equiv x A_{1} \ldots A_{n} \& x \text { universal in } \Gamma \\ \infty & \text { if } A \equiv x A_{1} \ldots A_{n} \& x \text { existential in } \Gamma \\ 1 & \text { if } A \equiv O \\ \max \left(\left\{1+\operatorname{ord}_{\Gamma}\left(A_{1}\right), \operatorname{ord}_{\Gamma}\left(A_{2}\right)\right\}\right) & \text { if } A \equiv A_{1} \rightarrow A_{2}\end{cases}$

The definition by cases is O.K. by Lemma 2.6. By convention, $\max (\{n, \infty\})=\infty$ and $n+\infty=\infty$. When $\Gamma$ is clear from the context, we simply speak about the order of $A$. Also if $Q x: A$ appear in $\Gamma$, then we sometimes speak about the order of $x$. For instance, if $\operatorname{ord}_{\Gamma}(\Gamma(x))=\infty$, then we say that $x$ is a variable of order $\infty$. Note that if $A$ is closed in $\Gamma$, then $\operatorname{ord}_{\Gamma}(A)$ is finite.

\section{Definition 3.2.}

1. A substitution is a finite set of triples $\left\langle x_{i} ; \gamma_{i} ; M_{i}\right\rangle$, such that the $x_{i}$ are pairwise distinct, $\gamma_{i}$ is an existential context and $\operatorname{dom}\left(\gamma_{i}\right)$ consists of fresh variables, possibly occurring in $M_{i}$. We let $\sigma, \sigma^{\prime}, \tau, \ldots$ range over substitutions.

2. If $\langle x ; \gamma ; M\rangle \in \sigma$, then we say that $\sigma$ binds $x$. Put $\operatorname{dom}(\sigma)=\{x \mid x$ bound by $\sigma\}$. $M$ is called a substitution term, $\gamma$ a substitution context. To indicate that the substitution context is an "auxiliary" context, we denote it by a small Greek letter.

3. A substitution $\sigma$ is extended to a function on pseudo-terms as follows:

$$
\begin{aligned}
\sigma(c) & =c \text { for } c \in \mathcal{C} \\
\sigma(x) & = \begin{cases}M & \text { if }\langle x ; \gamma ; M\rangle \in \sigma \\
x & \text { otherwise. }\end{cases} \\
\sigma\left(A_{1} A_{2}\right) & =\sigma\left(A_{1}\right) \sigma\left(A_{2}\right) \\
\sigma\left(\lambda x: A_{1} \cdot A_{2}\right) & =\lambda x: \sigma\left(A_{1}\right) \cdot \sigma\left(A_{2}\right) \\
\sigma\left(A_{1} \rightarrow A_{2}\right) & =\sigma\left(A_{1}\right) \rightarrow \sigma\left(A_{2}\right)
\end{aligned}
$$

If $\sigma=\left\{\left\langle x_{i} ; \gamma_{i} ; M_{i}\right\rangle \mid 1 \leq i \leq n\right\}$, then we sometimes write $\sigma(A)$ as $A\left[x_{1}:=M_{1}, \ldots, x_{n}:=M_{n}\right]$ or $A[\vec{x}:=\vec{M}]$.

4. A substitution $\sigma$ is extended to a function on pseudo-contexts as follows:

$$
\begin{aligned}
\sigma(\langle\rangle) & =\langle\rangle \\
\sigma(\Gamma, Q x: C) & = \begin{cases}\sigma(\Gamma), \gamma & \text { if } Q=\exists \text { and }\langle x ; \gamma ; M\rangle \in \sigma \\
\sigma(\Gamma), Q x: \sigma(C) & \text { otherwise. }\end{cases}
\end{aligned}
$$

5. Let $\Gamma$ be a legal context in $\lambda \circ$. Then we call $\sigma$ well-typed in $\Gamma$ when the following holds: 
(a) $\sigma$ binds no variables that are universal in $\Gamma$.

(b) $\sigma(\Gamma)$ is legal in $\lambda \circ$.

(c) For all existential variables $x$ in $\Gamma$ that are bound by $\sigma$ we have that $\sigma\left(\Gamma_{x}\right), \gamma \vdash_{\lambda \circ}$ $M: \sigma(\Gamma(x))$, where $\langle x ; \gamma ; M\rangle$ is the unique triple in $\sigma$ that binds $x$. In other words, we start with $\Gamma_{x} \vdash_{\lambda \circ} x: \Gamma(x)$ and end up with $\sigma\left(\Gamma_{x}\right) \vdash_{\lambda_{\circ}} \sigma(x): \sigma(\Gamma(x))$.

Note that the empty substitution, denoted by $\emptyset$, is well-typed in any legal context.

6. Let $\sigma$ and $\tau$ be substitutions. We define

$$
\sigma \circ \tau=\{\langle x ; \sigma(\gamma) ; \sigma(t)\rangle \mid\langle x ; \gamma ; t\rangle \in \tau\} \cup\{\langle x ; \gamma ; t\rangle \in \sigma \mid x \text { not bound by } \tau\}
$$

7. Let $\sigma$ be well-typed in some context $\Gamma$ which is legal in $\lambda \circ$ and suppose $\operatorname{dom}(\sigma) \subseteq$ $\operatorname{dom}(\Gamma)$. We can write $\sigma$ uniquely as $\sigma=\sigma_{\square} \cup \sigma_{*}$, where $\sigma_{s}$ is the set of triples $\langle x, \gamma, M\rangle$ such that $\Gamma_{x} \vdash_{\lambda \circ} \Gamma(x): s$. For a fixed sort $s, \sigma$ is said to be an $s$-substitution if for every triple $\langle x ; \gamma ; M\rangle$ we have $\Gamma_{x} \vdash_{\lambda \circ} \Gamma(x): s$. (Note that $\sigma_{\square}$ and $\sigma_{*}$ depend on $\Gamma$.)

Remark 3.3. Let $\Gamma$ be legal and $\sigma$ be well-typed in $\Gamma$. Then by inspection of the definition of substitutions and well-typedness one easily verifies that for all triples $\langle x ; \gamma ; t\rangle$ in $\sigma$ and all $y$ in $\operatorname{dom}(\sigma)$, we have: $y \notin F V(t)$. Given that contexts are assumed to be in the form described in Lemma 2.6 (8) and given Lemma 2.6 (3), it is no restriction to assume that a declaration of the form $x: A$ such that $A \in \mathcal{K}_{\square}$ only occurs in a substitution context in $\sigma_{\square}$ and that the substitution contexts in $\sigma_{\square}$ only contain such declarations. From now on we assume that substitutions satisfy this restriction. It is easy to check that the application of such substitutions to contexts of the form described in Lemma 2.6 (8) yields contexts of the same form.

\section{Definition 3.4.}

1. A unification problem in $\lambda \circ$ is a triple $\langle\Gamma ; A ; B\rangle$, where $\Gamma$ is a quantified context such that there exists a term $C$ such that $\Gamma \vdash_{\lambda_{0}} A: C$ and $\Gamma \vdash_{\lambda_{0}} B: C$. We assume that $\Gamma, A$ and $B$ are in LNF. If $\Gamma \vdash_{\lambda_{0}} C: *$, then we say that $\langle\Gamma ; A ; B\rangle$ is a unification problem for objects; if $\Gamma \vdash_{\lambda_{0}} C: \square$, then we say that $\langle\Gamma ; A ; B\rangle$ is a unification problem for types.

2. A matching problem in $\lambda \circ$ is a unification problem $\langle\Gamma ; A ; B\rangle$ in $\lambda \circ$ such that $B$ is closed in $\Gamma$. Note that this implies that $C$ is closed in $\Gamma$ (recall that $C$ is in LNF!).

3. A unification (or matching) problem $\langle\Gamma ; A ; B\rangle$ is of order $n$ if the types of the existential variables in $\Gamma$ have order at most $n$ in $\Gamma$. In the first five sections of this paper we assume that the type of every variable in $\Gamma$ is of finite order. In the appendix we show how the proof of Theorem 5.54 can be adapted to the general case. After Lemma 5.9 it is explained where the proof in Section 5 of this paper goes wrong if our restriction on the types of universal variables is relaxed. 
4. A solution for a unification problem $\langle\Gamma ; A ; B\rangle$ in $\lambda \circ$ is a substitution $\sigma$, well-typed in $\Gamma$, such that $\sigma(A)={ }_{\beta \eta} \sigma(B)$. (So a solution for a matching problem $\langle\Gamma ; A ; B\rangle$ in $\lambda \circ$ is a substitution $\sigma$, well-typed in $\Gamma$, such that $\sigma(A)={ }_{\beta \eta} B$.) $\sigma$ is called a solution for a collection of unification (matching) problems $\left\{P_{i} \mid i \in I\right\}$ if $\sigma$ is a solution for every $P_{i}$. By a standard argument, a set of unification (matching) problems $\left\{\left\langle\Gamma ; A_{i} ; B_{i}\right\rangle \mid 1 \leq i \leq n\right\}$ (for some $n \in \mathbb{N}$ ) can be encoded as a single unification (matching) problem. We call the unification (matching) problems in such a set $\Gamma$ compatible.

5. A solution $\sigma$ for a unification problem $\langle\Gamma ; A ; B\rangle$ in $\lambda \circ$ is closed when for all triples $\langle x ; \gamma ; M\rangle$ in $\sigma, \gamma \equiv\langle\rangle$ and $M$ is closed in $\sigma\left(\Gamma_{x}\right)$. Another option would be to say that a solution $\sigma$ is closed when we all substitution contexts in $\sigma$ are closed, whereas substitution terms need not be closed. The undecidability result involving closed solutions in Section 6 holds for both notions of a closed solution.

Now we give two examples of a third-order matching problem for objects in $\lambda \underline{\omega}$. The first example illustrates the use of new, existential variables in substitutions. The second example illustrates the difficulties which have to be overcome in order to prove the decidability of third-order matching in $\lambda \underline{\omega}$.

Example 3.5. We define $\left\langle\Gamma ; t_{1} ; t_{2}\right\rangle$, a third-order matching problem for objects in $\lambda \underline{\omega}$. Take

- $\Gamma \equiv\left\langle\forall A: *, \forall B: *, \forall g: B \rightarrow B \rightarrow B, \forall b_{1}: B, \forall b_{2}: B, \exists f:(A \rightarrow B) \rightarrow B\right\rangle$;

- $t_{1} \equiv g\left(f\left(\lambda x: A . b_{1}\right)\right)\left(f\left(\lambda x: A \cdot b_{2}\right)\right)$;

- $t_{2} \equiv g b_{1} b_{2}$.

One easily checks that every solution for this problem is of the form $\sigma=\{\langle f ;\langle\exists x: A\rangle ; \lambda y: A \rightarrow B . y x\rangle\}$,

for some existential variable $x$.

Example 3.6. We define $\left\langle\Gamma ; t_{1} ; t_{2}\right\rangle$, a third-order matching problem for objects in $\lambda \underline{\omega}$. Take

- $\Gamma \equiv\left\langle\forall B: *, \forall X: * \rightarrow *, \exists A_{1}: *, \exists A_{2}: *, \forall a_{1}:(X B), \forall a_{2}:(X B)\right.$, $\forall g:(X B) \rightarrow(X B) \rightarrow(X B) \rightarrow B, \exists y_{1}:\left(X A_{1}\right), \exists y_{2}:\left(X A_{1}\right)$, $\left.\exists f:\left(\left(X A_{2}\right) \rightarrow\left(X A_{2}\right) \rightarrow\left(X A_{2}\right)\right) \rightarrow\left(X A_{1}\right) \rightarrow\left(X A_{1}\right) \rightarrow(X B)\right\rangle$

- $t_{1} \equiv g$

$\left(f\left(\lambda x_{1}:\left(X A_{2}\right) \cdot \lambda x_{2}:\left(X A_{2}\right) \cdot x_{1}\right) y_{1} y_{2}\right)$

$\left(f\left(\lambda x_{1}:\left(X A_{2}\right) \cdot \lambda x_{2}:\left(X A_{2}\right) \cdot x_{2}\right) y_{1} y_{2}\right)$

$\left(f\left(\lambda x_{1}:\left(X A_{2}\right) \cdot \lambda x_{2}:\left(X A_{2}\right) \cdot x_{1}\right) y_{2} y_{1}\right)$

- $t_{2} \equiv g a_{1} a_{2} a_{2}$.

The reader is invited to check that this problem has a unique solution:

$$
\begin{aligned}
\sigma= & \left\{\left\langle A_{1} ;\langle\rangle ; B\right\rangle,\left\langle A_{2} ;\langle\rangle ; B\right\rangle,\left\langle y_{1} ;\langle\rangle ; a_{1}\right\rangle,\left\langle y_{2} ;\langle\rangle ; a_{2}\right\rangle,\right. \\
& \left.\left\langle f ;\langle\rangle ; \lambda z_{1}:(X B) \rightarrow(X B) \rightarrow(X B) . \lambda z_{2}:(X B) . \lambda z_{3}:(X B) . z_{1} z_{2} z_{3}\right\rangle\right\} .
\end{aligned}
$$


Note that this matching problem has as only solution a substitution which unifies the terms $X A_{1}$ and $X A_{2}$. For the proof of decidability of third-order matching in $\lambda \underline{\omega}$ it is essential that full (third-order) unification of types can be avoided. For it is in general undecidable whether a third-order unification problem for types has a solution or not (see [14], [4]). The idea is to avoid full unification of types by defining and solving matching problems for types in some specific order.

Let us take a closer look at how unification can be avoided in our example. The variable $f$ has three occurrences in $t_{1}$ and the term that is substituted for $f$ each time takes a term whose type is initially $\left(X A_{2}\right) \rightarrow\left(X A_{2}\right) \rightarrow\left(X A_{2}\right)$ and which after application to two arguments $\left(y_{1}, y_{2}\right.$ of type $\left.X A_{1}\right)$ should yield a term of type $X B$. So $X A_{2}$ has to be matched with $X B$. This constitutes a matching problem because $X B$ is closed. Thereafter we know that the type of the two arguments (i.e. $X A_{1}$ ) has to be matched with $X B$. This again constitutes a matching problem. Note that if we would first try to match $X A_{1}$ with $X A_{2}$ we would be faced with a unification problem.

The order is implemented as follows. First we define (and solve) matching problems that arise when we try to find terms that have to be substituted for those existential variables that have type $S_{1} \rightarrow \cdots \rightarrow S_{n} \rightarrow S$, where $S$ is closed. (In this case we start with $f$.) Then we apply the solutions for these matching problems to the types of the other existential variables (in this case $y_{1}$ and $y_{2}$ ), in the hope that the respective types (in this case $X A_{1}$ ) become closed (in this case the solution changes $X A_{1}$ to $X B$ ). If this hope is fulfilled then we define (and solve) the matching problems that arise when we try to find terms that have to be substituted for these variables w.r.t. their (new) types. For completeness we show that if the initial problem has a solution then the matching problems have a solution and all existential variables are treated (in case substituting a term for such a variable is essential to obtain a solution). We end this section by stating some properties of matching problems and substitutions.

Lemma 3.7. Let $P=\langle\Gamma ; A ; B\rangle$ be a matching problem in $\lambda \circ, \sigma$ a solution for $P$. If $x \in F V(A)$ is existential in $\Gamma$, then there exists a $y \in F V(A)$, bound by $\sigma$, of type $S_{1} \rightarrow \cdots \rightarrow S_{n} \rightarrow S$ in $\Gamma$ (for some $n \geq 0, S_{1}, \ldots, S_{n}, S$ ) such that $S$ is closed in $\Gamma$.

Proof. Induction on the LNF-structure of $A$.

\section{Lemma 3.8.}

1. Suppose $\Gamma \vdash_{\lambda_{\circ}} A: B$ and let $\sigma$ be well-typed in $\Gamma$. Then $\sigma(\Gamma) \vdash_{\lambda_{\circ}} \sigma(A): \sigma(B)$.

2. Suppose $\Gamma \vdash_{\lambda_{0}} A: s$ and let $\sigma$ be well-typed in $\Gamma$. Then $\operatorname{ord}_{\sigma(\Gamma)}(\sigma(A)) \leq \operatorname{ord}_{\Gamma}(A)$.

3. Let $\Gamma$ be legal, $\tau$ well-typed in $\Gamma$ and $\sigma$ well-typed in $\tau(\Gamma)$. Then $\sigma \circ \tau$ is well-typed in $\Gamma$ and $(\sigma \circ \tau)(\Gamma)=\sigma(\tau(\Gamma))$.

Proof. See [7].

Lemma 3.9. Let $P=\langle\Gamma ; A ; B\rangle$ be a matching problem of finite order in $\lambda \underline{\omega}$. If $P$ has solution $\sigma$ then it has a solution $\tau$ such that for every declaration $\exists x: C$ in a substitution context in $\tau, C$ is atomic and of order less than or equal to 2. 
Proof. Let $\exists x: C$ appear in a substitution context in $\sigma$. Write $C \equiv C_{1} \rightarrow \cdots \rightarrow C_{n} \rightarrow C^{\prime}$. By Remark A.4, we may assume that the order of $C$ is finite. Hence the order of $C^{\prime}$ is 1 or (if $\left.C^{\prime} \equiv *\right) 2$. Define $c_{x} \equiv \lambda x_{1}: C_{1} \ldots \lambda x_{n}: C_{n} . y$, where $y$ is a fresh variable of type $C^{\prime}$. Let $\sigma^{\prime}$ be the result of the following operation. Replace in the substitution context $\exists x: C$ by $\exists y: C^{\prime}$ and thereafter replace in all substitution terms and contexts $x$ by $c_{x}$. By the Substitution Lemma and the fact that $x$ does not occur in $\Gamma$ we have that $\sigma^{\prime}$ is well-typed in $\Gamma$. By Substitutivity, $\sigma^{\prime}(A) \rightarrow \beta \eta \operatorname{nf}(B)$. So $\sigma$ is a solution for $P$. By iterating this procedure, we obtain the required solution $\tau$.

\section{Lemma 3.10.}

1. Suppose that $\Gamma \vdash_{\lambda_{0}} A: B$ and $A$ is closed in $\Gamma$. Let $\sigma$ be a substitution, well-typed in $\Gamma$. Then $A$ is closed in $\sigma(\Gamma)$.

2. Let $\Gamma$ be legal in $\lambda \underline{\omega}$ and $\sigma$ well-typed in $\Gamma$. Suppose furthermore that $\sigma(\Gamma) \vdash_{\lambda \underline{\omega}} A: B:$ and that $A$ is closed in $\sigma(\Gamma)$. Then $A$ is closed in $\Gamma$.

3. Let $\Gamma$ be legal in $\lambda_{\circ}$ and $\sigma$ well-typed in $\Gamma$. Then $\sigma_{\square}$ is well-typed in $\Gamma$ and $\sigma_{*}$ is well-typed in $\sigma_{\square}(\Gamma)$.

4. Suppose $\Gamma \vdash_{\lambda \circ} A: B$ and $\sigma$ is well-typed in $\Gamma$. Then $\sigma_{*}\left(\sigma_{\square}(A)\right) \equiv \sigma(A)$.

5. Let $\Gamma$ be legal in $\lambda \circ$ and $\sigma$ well-typed in $\Gamma$. Suppose $\sigma(\Gamma) \vdash_{\lambda \circ} A: B$ and $A$ is closed in $\sigma(\Gamma)$. Then already $\sigma_{\square}(\Gamma) \vdash_{\lambda_{\circ}} A: B$ and $A$ is closed in $\sigma_{\square}(\Gamma)$.

Proof. For (1): By Lemma $3.8(1)$ and the fact that $\sigma(A) \equiv A$, we have that $\sigma(\Gamma) \vdash_{\lambda \underline{\omega}} A$ : $\sigma(B)$. By induction on $\operatorname{lh}(\Gamma)+\operatorname{lh}(A)$ one proves that $A$ is closed in $\sigma(\Gamma)$. The proofs of $(2)$, (4), (5) are straightforward inductions on the structure of $A$. The proof of (3) is an induction on the length of $\Gamma$.

The previous lemma allows the following useful notation. Suppose $\Gamma \vdash_{\lambda_{0}} A: B$ and let $\sigma$ be well-typed in $\Gamma$. Write $\sigma_{\square}$ as $\left\{\left\langle x_{i} ; \gamma_{i} ; S_{i}\right\rangle \mid 1 \leq i \leq n\right\}$ and $\sigma_{*}$ as $\left\{\left\langle y_{i} ; \delta_{i} ; T_{i}\right\rangle \mid 1 \leq i \leq m\right\}$. Recall that we sometimes write $\sigma(A)$ as

$$
A\left[x_{1}:=S_{1}, \ldots, x_{n}:=S_{n}, y_{1}:=T_{1}, \ldots, y_{m}:=T_{m}\right] .
$$

By Lemma $3.10(3),(4)$, we can also write $\sigma(A)$ as

$$
A\left[x_{1}:=S_{1}, \ldots, x_{n}:=S_{n}\right]\left[y_{1}:=T_{1}, \ldots, y_{m}:=T_{m}\right],
$$

where $A\left[x_{1}:=S_{1}, \ldots, x_{n}:=S_{n}\right]$ is legal in $\sigma_{\square}(\Gamma)$ and hence in $\sigma(\Gamma)$.

Lemma 3.11. Suppose $\Gamma \vdash_{\lambda \circ} A: B$. Suppose that $B$ is closed in $\Gamma$ and that for every $x \in F V(A)$, universal in $\Gamma$ such that $\Gamma(x)$ is non-atomic, $\Gamma(x)$ is closed in $\Gamma$. Let $\sigma$ be well-typed in $\Gamma$ and suppose that $\sigma(A)$ is closed in $\sigma(\Gamma)$. Suppose $z \in F V(A)$ is existential in $\Gamma$ and of type $Z_{1} \rightarrow \cdots \rightarrow Z_{k} \rightarrow Z$, where $Z$ is not closed in $\Gamma$. For every occurrence of $z$ there exists a $u$, bound by $\sigma$, such that, for some terms $D_{1}, \ldots, D_{l}$, the term $u D_{1} \ldots D_{l}$ has an occurrence in $A$ and, for some $1 \leq j \leq l$, this occurrence of $z$ is an occurrence in $D_{j}$. 
Proof. By induction on the LNF-structure of $A$. Write $A \equiv \lambda x_{1}: S_{1} \ldots \lambda x_{n}: S_{n} . y t_{1} \ldots t_{m}$. We distinguish two cases. The first case is where $y$ is universal in $\Gamma$ or an $x_{i}(1 \leq i \leq n)$. The result follows easily from the induction hypothesis. The second case is where $y$ is existential in $\Gamma$. We can take $y$ for the required variable $u$.

\section{Third-order matching for types}

As explained in the previous section, the proof of decidability of third-order matching for objects hinges on the possibility to avoid having to solve (third-order) unification problems for types. The strategy is to decompose these unification problems into third-order matching problems for types. This of course only makes sense if it is indeed decidable whether a thirdorder matching problem for types in $\lambda \underline{\omega}$ has a solution or not. In this section we will, without going into any detail, show that this is the case. The proof of this fact uses a translation ()$^{-}$from [13] which maps types and constructors from $\lambda \underline{\omega}$ to objects of $\lambda \tau$. This translation preserves $\beta \eta$-reduction and (after extension to contexts) judgements. It is easy to extend this translation further to substitutions and to define a left inverse ()$^{+}$to this translation (up to $\beta$-conversion). This enables us to prove the following result.

Theorem 4.1. Let $P_{1}=\langle\Gamma ; A ; B\rangle$ be a matching problem of order $n$ for types in $\lambda \underline{\omega}$. Then $P_{2}=\left\langle(\Gamma)^{-} ;(A)^{-} ;(B)^{-}\right\rangle$is a matching problem of order $n-1$ in $\lambda \tau$ and $\sigma$ is a solution for $P_{1}$ iff $(\sigma)^{-}$is a solution for $P_{2}$.

Corollary 4.2. It is decidable whether a third-order matching problem for types in $\lambda \underline{\omega}$ has a solution or not.

Proof. It is decidable whether a second-order matching problem in $\lambda \tau$ has a solution; see $[7]$.

\section{$5 \quad$ Third-order matching for objects}

In this section we will show that it is decidable whether a third-order matching problem for objects in $\lambda \underline{\omega}$ has a solution. First we define a translation that maps such a problem $P=\langle\Gamma ; A ; B\rangle$ to a third-order matching problem $|P|$ in $\lambda \tau$ and solutions $\sigma$ for $P$ to solutions $|\sigma|$ for $|P|$. Then we will prove that a substitution $\sigma$ is a solution for $P$ iff $\sigma$ is well-typed in $\Gamma$ and $|\sigma|$ is a solution for $|P|$. This divides the task of finding solutions for $P$ in two parts: find solutions $\tau$ for $|P|$ and see if we can "lift" such solutions to substitutions $\tau^{\prime}$ that are well-typed in $\Gamma$ and such that $\left|\tau^{\prime}\right|=\tau$. Dowek has shown (in [8]) that to find solutions for $|P|$ it does no harm to restrict one's attention to a search space whose cardinality is bounded by a function value depending only on the size of $|P|$. Given such a solution $\tau$, we will try to lift $\tau$ in two stages. First we decorate $\tau$ in a straightforward way: given an existential variable $x$ of type $S_{1} \rightarrow \cdots \rightarrow S_{n} \rightarrow S$ in $\Gamma$ and a triple $\langle x ; \gamma ; t\rangle$ in $\tau$, where $t \equiv \lambda x_{1}:\left|S_{1}\right| \ldots \lambda x_{n}:\left|S_{n}\right| . y t_{1} \ldots t_{m}$, we decorate $t$ to $\lambda x_{1}: S_{1} \ldots \lambda x_{n}: S_{n} . y t_{1}^{\prime} \ldots t_{m}^{\prime}$, where $t_{j}^{\prime}$ is the decorated version of $t_{j}$. This procedure need not yield terms that are well-typed in $\lambda \underline{\omega}$. In order to change these terms to 
well-typed ones, we define (starting from $\Gamma$ and $\tau$ ) a third-order matching problem Match for types in $\lambda \underline{\omega}$ such that if this problem has a solution $\rho$ then the composition of $\rho$ with the decorated substitution is a substitution $\theta$ that is well-typed in $\Gamma$ and such that $|\theta|=\tau$ (hence $|\theta|$ is a solution for $|P|$ and $\theta$ is a solution for $P$ ).

\subsection{Flattening types}

We define a map, $|\cdot|$, that replaces all atomic subtypes by $O$. This map is extended to contexts and substitutions. We show that it preserves judgements, order, $\beta \eta$-reduction, the property of being a matching problem of finite order and the property of being a solution for such a problem. When we say below that a term is in (D-) HNF, then this term is not assumed to be normal or in LNF.

\section{Definition 5.1.}

1. Suppose $\Gamma \vdash_{\lambda_{0}} A: s$, where $A$ is in HNF. We define $|A|_{T}$ by induction on the structure of $A$.

$$
\begin{aligned}
|*|_{T} & =O \\
|O|_{T} & =O \\
\left|x A_{1} \cdots A_{n}\right|_{T} & =O \\
\left|A_{1} \rightarrow A_{2}\right|_{T} & =\left|A_{1}\right|_{T} \rightarrow\left|A_{2}\right|_{T}
\end{aligned}
$$

By inspecting Definition 2.9, one easily verifies that the case distinction is O.K.

2. Let $\Gamma$ be legal in $\lambda \circ$ (and in HNF) and suppose that for every domain $D$ in $A$ we have $\Gamma \vdash^{\lambda \underline{\omega}} D: s$ (and $D$ is in HNF). We define $|A|$ by induction on the structure of $A$.

$$
\begin{aligned}
|c| & =c \quad \text { for } c \in \mathcal{C} \\
|x| & =x \\
\left|A_{1} A_{2}\right| & =\left|A_{1}\right|\left|A_{2}\right| \\
\left|\lambda x: A_{1} \cdot A_{2}\right| & =\lambda x:\left|A_{1}\right|_{T} \cdot\left|A_{2}\right| \\
\left|A_{1} \rightarrow A_{2}\right| & =\left|A_{1}\right| \rightarrow\left|A_{2}\right|
\end{aligned}
$$

3. We extend the definition to contexts in HNF that are legal in $\lambda \circ$.

$$
\begin{aligned}
|\langle\rangle| & =\langle\rangle \\
|\Gamma, Q x: A| & =|\Gamma|, Q x:|A|_{T} \text { if } \Gamma \vdash_{\lambda_{\circ}} A: * \\
|\Gamma, Q x: A| & =|\Gamma| \text { if } \Gamma \vdash_{\lambda \circ} A: \square
\end{aligned}
$$

4. Let $\Gamma$ be legal in $\lambda \circ, \sigma$ well-typed in $\Gamma$ and $\operatorname{dom}(\sigma) \subseteq \operatorname{dom}(\Gamma)$.

Then $|\sigma|=\left\{\langle x ;|\gamma| ;|S|\rangle \mid\langle x ; \gamma ; S\rangle \in \sigma_{*}\right\}$. (We need to have $\operatorname{dom}(\sigma) \subseteq \operatorname{dom}(\Gamma)$, because otherwise $\sigma_{*}$ is not defined.) 
It is easily seen that if $\Gamma \vdash_{\lambda \tau} A: B, \sigma$ is well-typed in $\Gamma$ and $\operatorname{dom}(\sigma) \subseteq \operatorname{dom}(\Gamma)$, then $|\Gamma| \equiv \Gamma,|A| \equiv A,|B|_{T} \equiv B$ and $|\sigma| \equiv \sigma$. Because of this, all results listed below are trivially true when we replace $\lambda \underline{\omega}$ by $\lambda \tau$.

Lemma 5.2. Suppose $\Gamma \vdash_{\lambda \underline{\omega}} A: B$, where $\Gamma$ and $A$ are in (D-) HNF.

1. $|A|$ is in D-HNF.

2. If $A$ is normal (resp. in LNF), then $|A|$ is normal (resp. in LNF).

3. If $x \in F V(|A|)$, then $x \in F V(A)$.

The same holds for $|A|_{T}$ (in case $B \equiv s$ ).

Proof. (1), (2), (3): by induction on the structure of $A$.

\section{Lemma 5.3.}

1. Suppose that $\Gamma \vdash_{\lambda \underline{\omega}} A: s$ and that $\Gamma$ and $A$ are in HNF. Then $|\Gamma| \vdash_{\lambda \tau}|A|_{T}: *$.

2. Suppose that $\Gamma \vdash_{\lambda \underline{\omega}} A: B: *$ and $\Gamma, A$ and $B$ are in (D-) HNF. Then $|\Gamma| \vdash_{\lambda \tau}|A|$ : $|B|_{T}: *$.

\section{Proof.}

1. By induction, first on the length of $\Gamma$ and second on the HNF-structure of $A$ and using Lemma 2.6, one proves that $|\Gamma|$ is legal in $\lambda \tau$ and $|A| \in \mathcal{K}_{*}$. The result then follows from Lemma $2.6(2)$.

2. By induction on the structure of $A$, using (1).

Lemma 5.4. Suppose that $\Gamma \vdash_{\lambda \underline{\omega}} A: *$, where $\Gamma$ and $A$ are in $\operatorname{HNF}$ and $\operatorname{ord}_{\Gamma}(A)$ is finite. Then $\operatorname{ord}_{\Gamma}(A)=\operatorname{ord}_{|\Gamma|}\left(|A|_{T}\right)$.

Proof. By Lemma 5.2 and Lemma $5.3(1) \operatorname{ord}_{|\Gamma|}\left(|A|_{T}\right)$ is defined. The proof of the lemma proceeds by induction on the HNF-structure of $A$.

Corollary 5.5. Let $P=\langle\Gamma ; A ; B\rangle$ be a matching problem of order $n$ for objects in $\lambda \underline{\omega}$. Then $\langle|\Gamma| ;|A| ;|B|\rangle$ is a matching problem of order $n$ in $\lambda \tau$. We denote it by $|P|$.

Proof. By Lemma 5.2 (2), Lemma 5.3 (2) and Lemma 5.4. 
Lemma 5.6. Assume that all terms mentioned in the premises of (1) and (2) are in (D-) HNF.

1. Suppose $\Gamma, \exists x: C, \Delta \vdash_{\lambda \underline{\omega}} A: *$. Suppose $\Gamma \vdash_{\lambda \underline{\omega}} D: C$ and $\operatorname{ord}_{\Gamma}(A)$ is finite. Then $A[x:=D]$ is in $\mathrm{HNF}$.

2. Suppose $\Gamma, Q x: C, \Delta \vdash_{\underline{\lambda}} A: B: *$ and $\Gamma \vdash_{\lambda \underline{\omega}} D: C: *$. Then $A[x:=D]$ is in D-HNF.

Proof. (1), (2): by induction on the (HNF-) structure of $A$. The intuition for (1) is as follows. $A$ is composed of arrows and atomic types $y A_{1} \ldots A_{n}$. Since the order of $A$ is finite, $y$ will not be existential in $\Gamma$, hence will not be $x$.

In other words, $\left(y A_{1} \ldots A_{n}\right)[x:=D] \equiv y\left(A_{1}[x:=D]\right) \ldots\left(A_{n}[x:=D]\right)$. The intuition for $(2)$ is that by Lemma $2.6(3), x$ does not occur in domains in $A$. Hence the substitution of $D$ for $x$ does not affect the domains in $A$.

Lemma 5.7. Assume that all terms mentioned in the premises of (1) and (2) are in (D-) HNF.

1. Suppose $\Gamma, Q x: C, \Delta \vdash^{\underline{\underline{\omega}}} A: B: *$ and $\Gamma \vdash_{\lambda \underline{\omega}} D: C: *$. Then $|A[x:=D]| \equiv|A|[x:=$ $|D|]$.

2. Suppose $\Gamma, \exists x: C, \Delta \vdash_{\lambda \underline{\omega}} A: *$, where $\operatorname{ord}_{\Gamma}(A)$ is finite. Suppose $\Gamma \vdash_{\lambda \underline{\omega}} B: C$. Then $|A[x:=B]|_{T} \equiv|A|_{T}$.

\section{Proof.}

1. By induction on the structure of $A$. The same intuition as for Lemma 5.6 (2) applies.

2. By Lemma $5.6(1),|A[x:=B]|_{T}$ is defined, since $A[x:=B]$ is in HNF. The proof proceeds by induction on the HNF-structure of $A$. The same intuition as for Lemma 5.6 (1) applies.

Lemma 5.8. Suppose $\Gamma \vdash_{\lambda \underline{\omega}} A: B: *$, where $\Gamma$ and $A$ are in (D-) HNF. Then

$$
\left(A \rightarrow \beta \eta A^{\prime}\right) \Rightarrow|A| \rightarrow \beta \eta\left|A^{\prime}\right| .
$$

Proof. By induction on the generation of $\rightarrow_{\beta \eta}$, using Lemma 5.7.

The following lemma states that the application of substitutions to terms, types and contexts under certain conditions commutes with the $\|$-function. Its validity hinges on our restriction w.r.t. the types of universal variables and the fact that substitutions are (w.l.o.g.) assumed to satisfy the condition in Remark 3.3 .

Lemma 5.9. Suppose $\Gamma \vdash_{\lambda \circ} A: B: *$, where $\Gamma$ and $A$ are in (D-) HNF. Suppose that for all $y \in \operatorname{dom}(\Gamma), \Gamma(y)$ is of finite order and that every domain in $A$ has finite order w.r.t. $\Gamma$. Let $\sigma$ be well-typed in $\Gamma, \operatorname{dom}(\sigma) \subseteq \operatorname{dom}(\Gamma)$. Then $|\sigma(\Gamma)| \equiv|\sigma|(|\Gamma|)$, and $|\sigma(A)| \equiv|\sigma|(|A|)$. If $B$ is of finite order, then $|\sigma(B)| \equiv|\sigma|(|B|) \equiv|B|$.

Proof. By induction on the length of $\Gamma$, the structure of $A$, and the HNF-structure of $B$ respectively. Use Lemma 5.7. 
In the presence of universal variables of order $\infty$ there may occur situations where the condition on the domains in the previous lemma is not met, thereby preventing the application of that lemma. Let us see why. Let $\Gamma \equiv\langle\forall B: *, \exists X: * \rightarrow *, \forall x:(X B \rightarrow X B) \rightarrow B\rangle$ and let $A$ consist of the variable $x$. In LNF, $A \equiv \lambda y: X B \rightarrow X B \cdot x(\lambda z: X B . y z)$. Note that $x$ is a variable of order $\infty$ and $X B$ is a domain of order $\infty$ in $A$. Let $\sigma$ be $\{\langle X ;\langle\rangle ; \lambda \beta: *$ $. \beta \rightarrow \beta\rangle\}$. We have that $|\sigma|=\emptyset$ and $|\sigma|(|A|) \equiv|A| \equiv \lambda y: O \rightarrow O \cdot x(\lambda z: O . y z)$. Furthermore we have that (in LNF) $\sigma(A) \equiv \lambda y:(B \rightarrow B) \rightarrow B \rightarrow B \cdot x(\lambda z: B \rightarrow B \cdot y(\lambda u: B . z u)$ ) and $|\sigma(A)| \equiv$ $\lambda y:(O \rightarrow O) \rightarrow O \rightarrow O . x(\lambda z: O \rightarrow O . y(\lambda u: O . z u))$. So $|\sigma|(|A|) \not \equiv|\sigma(A)|$.

Lemma 5.10. Suppose that $\Gamma \vdash_{\lambda \underline{\omega}} \lambda x_{1}: S_{1} \ldots \lambda x_{n}: S_{n} . x t_{1} \ldots t_{m}: S$, where $S_{i}(1 \leq i \leq n)$ and $\Gamma(y)$ (for all $y \in F V\left(\lambda x_{1}: S_{1} \ldots \lambda \bar{x}_{n}: S_{n} . x t_{1} \ldots t_{m}\right)$ ) are of finite order w.r.t. $\Gamma$. Then every domain in $\lambda x_{1}: S_{1} \ldots \lambda x_{n}: S_{n} . x t_{1} \ldots t_{m}$ is of finite order w.r.t. $\Gamma$.

Proof. Induction on the LNF-structure of $\lambda x_{1}: S_{1} \ldots \lambda x_{n}: S_{n} . x t_{1} \ldots t_{m}$.

Proposition 5.11. Let $P_{1}=\langle\Gamma ; A ; B\rangle$ be a matching problem of order $n$ for objects in $\lambda \underline{\omega}, \sigma$ a solution for $P_{1}$ and suppose $\operatorname{dom}(\sigma) \subseteq \operatorname{dom}(\Gamma)$. Then $|\sigma|$ is a solution for $P_{2}=$ $\langle|\Gamma| ;|A| ;|B|\rangle$.

Proof. Write $\sigma_{\square}=\left\{\left\langle x_{i} ; \delta_{i} ; S_{i}\right\rangle \mid 1 \leq i \leq n\right\}$ and $\sigma_{*}=\left\{\left\langle y_{i} ; \gamma_{i} ; T_{i}\right\rangle \mid 1 \leq i \leq m\right\}$. Then $A[\vec{x}:=\vec{S}][\vec{y}:=\vec{T}]={ }_{\beta \eta} B$ and so by Confluence there exists a term $C$ such that

$$
A[\vec{x}:=\vec{S}][\vec{y}:=\vec{T}] \rightarrow_{\beta \eta} C \text { and } B \rightarrow_{\beta \eta} C .
$$

We need to show that $|A|[\vec{y}:=|\vec{T}|]={ }_{\beta \eta}|B|$. By Lemma 5.8 we have that $|B| \rightarrow_{\beta \eta}|C|$. So we are done if we can show that $|A|[\vec{y}:=|\vec{T}|] \rightarrow \beta \eta|C|$. We calculate:

$$
\begin{array}{ll}
A[\vec{x}:=\vec{S}][\vec{y}:=\vec{T}] \rightarrow_{\beta \eta} C & \text { hence by Lemma } 5.10 \text {, Lemma } 5.8 \text { and Lemma } 5.6 \\
|A[\vec{x}:=\vec{S}][\vec{y}:=\vec{T}]| \rightarrow \rightarrow_{\beta \eta}|C| & \text { hence by Lemma } 5.7 \text { (1) and well-typedness of } \sigma \\
|A[\vec{x}:=\vec{S}]|[\vec{y}:=|\vec{T}|] \rightarrow \beta \eta|C| & \text { hence by Lemma } 5.7 \text { (2) } \\
|A|[\vec{y}:=|\vec{T}|] \rightarrow \beta \eta|C| . &
\end{array}
$$

Note that Lemma 5.10 is applicable because the type of $A$ is closed and hence of finite order in $\Gamma$. Next we show that $|\sigma|$ is well-typed in $|\Gamma|$. First note that $|\sigma|(|\Gamma|)$ is legal in $\lambda \tau$ by Lemma 5.9. Suppose $x$ is bound by $|\sigma|$ (hence by $\sigma$ ). Let $\Gamma$ be $\Delta, \exists x: D, \Delta^{\prime}$. Then $|\Gamma| \equiv|\Delta|, \exists x:|D|,\left|\Delta^{\prime}\right|$. Say that $\langle x ; \gamma ; S\rangle \in \sigma$. We have $\sigma(\Delta), \gamma \vdash_{\lambda \omega} S: \sigma(D)$ and so (by Lemma 5.6 (1), Lemma 5.3 (2), Lemma 5.7 (2) and Lemma 5.9), $|\sigma|(|\overline{\mid}|),|\gamma| \vdash_{\lambda \tau}|S|:|D|_{T}$. Thus $|\sigma|$ is well-typed in $|\Gamma|$. We conclude that $|\sigma|$ is a solution for $P_{2}$.

Lemma 5.12. Suppose $\Gamma \vdash_{\lambda \underline{\omega}} A_{1}: B: *$ and $\Gamma \vdash_{\lambda \underline{\omega}} A_{2}: B: *$. If $\Gamma, A_{1}, A_{2}$ and $B$ are normal or in LNF and $\left|A_{1}\right| \equiv\left|A_{2}\right|$, then $A_{1} \equiv A_{2}$.

Proof. By induction on the LNF-structure of $A_{1}$. Use the fact that domains are syntactically equal since $A_{1}$ and $A_{2}$ have the same type $B$ and $B$ is normal (or in LNF). 
The following lemma is a key step in the main proof. It allows us to cut up the problem of finding solutions for third-order matching problems in $\lambda \underline{\omega}$ into two relatively easy subproblems.

Lemma 5.13. Let $P=\langle\Gamma ; A ; B\rangle$ be a third-order matching problem for objects in $\lambda \underline{\omega}$. Let $\sigma$ be a substitution, well-typed in $\Gamma, \operatorname{dom}(\sigma) \subseteq \operatorname{dom}(\Gamma)$. Suppose that $|\sigma|$ is a solution for $|P|$. Then $\sigma$ is a solution for $P$.

Proof. It suffices to show that $\sigma(A) \rightarrow \beta \eta \operatorname{nf}(B)$. Since $\sigma$ is well-typed in $\Gamma, \operatorname{nf}(\sigma(A))$ exists. We have $\sigma(A) \rightarrow \beta \eta \operatorname{nf}(\sigma(A))$. As in the proof of Proposition 5.11, we have $|\sigma(A)| \rightarrow \beta \eta$ $|\operatorname{nf}(\sigma(A))|$ and by Lemma 5.2 we know that $|\operatorname{nf}(\sigma(A))|$ is normal. By Lemma 5.9 we know $|\sigma|(|A|) \rightarrow_{\beta \eta}|\operatorname{nf}(\sigma(A))|$. Since $|\sigma|$ is a solution for $|P|$, we have that $|\sigma|(|A|) \rightarrow_{\beta \eta} \operatorname{nf}(|B|)$. As before, $\operatorname{nf}(|B|) \equiv|\operatorname{nf}(B)|$. We obtain that $|\operatorname{nf}(\sigma(A))| \equiv|\operatorname{nf}(B)|$. Furthermore, $B$ and $\sigma(A)$ have the same type, hence, by Subject Reduction, $\operatorname{nf}(\sigma(A))$ and $\operatorname{nf}(B)$ have the same type. By Lemma 5.12, we infer that $\operatorname{nf}(\sigma(A)) \equiv \operatorname{nf}(B)$. We conclude that $\sigma$ is a solution for $P$. $\quad \square$

\subsection{Decompositions and standard solutions}

In [8], Dowek defines for each third-order matching problem $P$ in $\lambda \tau$ and each solution $\sigma$ for $P$, a set $\Phi(P, \sigma)$ of third-order matching problems in $\lambda \tau$. This set is such that for each problem $\langle\Gamma ; A ; B\rangle$ in $\Phi(P, \sigma), A$ is of the form $x A_{1} \ldots A_{n}$, where $x$ is existential in $\Gamma$ (we say that $x$ is a head in $\Phi(P, \sigma)) . \sigma$ is a solution for $\Phi(P, \sigma)$ and each solution for $\Phi(P, \sigma)$ is also a solution for $P$. Using this set, $\sigma$ is transformed to $\sigma^{\prime}$, a substitution which is also a solution for $P$ but more efficient in two ways. First, the substitution terms in $\sigma^{\prime}$ are stripped of irrelevant parts and second, $\sigma^{\prime}$ does not contain irrelevant triples. Using this efficiency, Dowek shows that it is decidable whether a third-order matching problem in $\lambda \tau$ has a solution or not.

In this section we present a slight generalisation of this "decomposition" to the setting of $\lambda \underline{\omega}$. The generalisation is slight in the sense that we do not claim that a solution for the decomposition is a solution for the initial problem. It would not be difficult to adapt the definition to meet this additional requirement, but since this property is not used in the sequel we have chosen not to do so. The generalisation is instrumental in proving the completeness of the algorithm we define to establish decidability of third-order matching for objects in $\lambda \underline{\omega}$.

From this point on we use the following convention. If $\Gamma \vdash_{\lambda_{0}} A: B$ and $\sigma$ is well-typed in $\Gamma$, then we write $\sigma(A)$ for $\operatorname{lnf}_{\sigma(\Gamma)}(\sigma(A))$. Next we state an important technical lemma.

Lemma 5.14. Suppose $P=\left\langle\Gamma ; x A_{1} \ldots A_{n} ; B\right\rangle$ is a third-order matching problem for objects in $\lambda \circ$, where $x$ is existential in $\Gamma$; say $\exists x: S_{1} \rightarrow \cdots \rightarrow S_{n} \rightarrow S$ occurs in $\Gamma$. Fix $1 \leq i \leq n$. Write $S_{i} \equiv R_{1} \rightarrow \cdots \rightarrow R_{m} \rightarrow R$. Suppose $\sigma$ is a solution for $P$. Write $A_{i} \equiv$ $\lambda y_{1}: R_{1} \ldots \lambda y_{m}: R_{m} . A_{i}^{\prime}$ and put $\Delta \equiv \sigma(\Gamma), \forall y_{1}: \sigma_{\square}\left(R_{1}\right), \ldots, \forall y_{m}: \sigma_{\square}\left(R_{m}\right)$. Consider the normal form of $\sigma(x) \sigma\left(A_{1}\right) \ldots \sigma\left(A_{i-1}\right) z_{i} \sigma\left(A_{i+1}\right) \ldots \sigma\left(A_{n}\right)$, where $z_{i}$ is a fresh variable of type $\sigma_{\square}\left(S_{i}\right)$. Suppose $z_{i}$ occurs in this normal form. Then $P^{\prime}=\left\langle\Delta ; \sigma_{\square}\left(A_{i}^{\prime}\right) ; \sigma\left(A_{i}^{\prime}\right)\right\rangle$ is a thirdorder matching problem for objects in $\lambda \circ$. Moreover $\sigma_{*}$ is a solution for $P^{\prime}$.

Proof. The first point follows from the following observations. 
1. By Lemma 3.9, every variable added to $\Gamma$ by $\sigma_{\square}$ has order 2 and by Lemma 3.8, the order of every existential variable in $\sigma_{\square}(\Gamma)$ has order less than or equal to 3 in $\sigma_{\square}(\Gamma)$.

2. $\Gamma \vdash_{\lambda \circ} A_{i}: S_{i} \Rightarrow \Gamma, \forall y_{1}: R_{1}, \ldots, \forall y_{m}: R_{m} \vdash_{\lambda \circ} A_{i}^{\prime}: R \Rightarrow$ (by Lemma 3.8 (1) and Lemma $3.10(3)) \sigma_{\square}(\Gamma), \forall y_{1}: \sigma_{\square}\left(R_{1}\right), \ldots, \forall y_{m}: \sigma_{\square}\left(R_{m}\right) \vdash_{\lambda \circ} \sigma_{\square}\left(A_{i}^{\prime}\right): \sigma_{\square}(R)$.

3. $\Gamma, \forall y_{1}: R_{1}, \ldots, \forall y_{m}: R_{m} \vdash_{\lambda \circ} A_{i}^{\prime}: R \Rightarrow$ (by Lemma $\left.3.8(1)\right) \sigma\left(\Gamma, \forall y_{1}: R_{1}, \ldots, \forall y_{m}:\right.$ $\left.R_{m}\right) \vdash_{\lambda_{\circ}} \sigma\left(A_{i}^{\prime}\right): \sigma(R) \Leftrightarrow \sigma(\Gamma), \forall y_{1}: \sigma_{\square}\left(R_{1}\right), \ldots, \forall y_{m}: \sigma_{\square}\left(R_{m}\right) \vdash_{\lambda_{\circ}} \sigma\left(A_{i}^{\prime}\right): \sigma_{\square}(R)$.

4. By Lemma 2.11, $\sigma\left(A_{i}^{\prime}\right)$ is closed in $\sigma(\Gamma), \forall y_{1}: \sigma_{\square}\left(R_{1}\right), \ldots, \forall y_{m}: \sigma_{\square}\left(R_{m}\right)$. (Apply this lemma with $\sigma(\Gamma)$ for $\Gamma, z_{i}$ for $x,\langle\rangle$ for $\Delta, \sigma(x) \sigma\left(A_{1}\right) \ldots \sigma\left(A_{i-1}\right) z_{i} \sigma\left(A_{i+1}\right) \ldots \sigma\left(A_{n}\right)$ for $A, \sigma\left(A_{i}\right)$ for $B$, and the normal form of $B$ for $C$.)

5. $\sigma\left(A_{i}^{\prime}\right)$ closed in $\sigma(\Gamma), \forall y_{1}: \sigma_{\square}\left(R_{1}\right), \ldots, \forall y_{m}: \sigma_{\square}\left(R_{m}\right) \Rightarrow$ (by Lemma $3.10(5)$ )

$\sigma_{\square}(\Gamma), \forall y_{1}: \sigma_{\square}\left(R_{1}\right), \ldots, \forall y_{m}: \sigma_{\square}\left(R_{m}\right) \vdash_{\lambda \circ} \sigma\left(A_{i}^{\prime}\right): \sigma_{\square}(R)$.

Moreover $\sigma\left(A_{i}^{\prime}\right)$ is closed in $\sigma_{\square}(\Gamma), \forall y_{1}: \sigma_{\square}\left(R_{1}\right), \ldots, \forall y_{m}: \sigma_{\square}\left(R_{m}\right)$.

The second point follows from Lemma 3.10 (3), (4).

Definition 5.15. Let $P=\langle\Gamma ; A ; B\rangle$ be a third-order matching problem in $\lambda \circ$ and let $\sigma$ be a solution for $P$. By induction on the length of $\sigma_{\square}(A)$ we define $\Phi(P, \sigma)$, a tree in which some of the nodes are labeled with triples (these triples can be proved to be third-order matching problems). It suffices to distinguish the following cases.

- $A \equiv x A_{1} \ldots A_{n}$, where $x$ is universal in $\Gamma$. Because $\sigma$ is a solution for $P, B \equiv x B_{1} \ldots B_{n}$ and $\sigma$ is a solution for $\left\langle\Gamma ; A_{i} ; B_{i}\right\rangle$, for every $1 \leq i \leq n$. We let $\Phi(P, \sigma)$ consist of an unlabeled root and subtrees $\Phi\left(\left\langle\Gamma ; A_{1} ; B_{1}\right\rangle, \sigma\right), \ldots, \Phi\left(\left\langle\Gamma ; A_{n} ; B_{n}\right\rangle, \sigma\right)$ (ordered from left to right).

- $A \equiv \lambda x: A_{1} \cdot A_{2}$. Again because $\sigma$ is a solution for $P, B \equiv \lambda x: A_{1} . B_{2}$ and $\sigma$ is a solution for $\left\langle\Gamma, \forall x: A_{1} ; A_{2} ; B_{2}\right\rangle$. Put $\Phi(P, \sigma)=\Phi\left(\left\langle\Gamma, \forall x: A_{1} ; A_{2} ; B_{2}\right\rangle, \sigma\right)$.

- $A \equiv x A_{1} \ldots A_{n}$, where $x$ is existential in $\Gamma$. Suppose $\exists x: S_{1} \rightarrow \cdots \rightarrow S_{n} \rightarrow S$ occurs in $\Gamma$. Put $\Delta:=\sigma_{\square}(\Gamma)$. For all $i, 1 \leq i \leq n$, write $A_{i} \equiv \lambda y_{1}: R_{1} \ldots \lambda y_{m}: R_{m} . A_{i}^{\prime}$ and consider the normal form of $\sigma(x) \sigma\left(A_{1}\right) \ldots \sigma\left(A_{i-1}\right) z_{i} \sigma\left(A_{i+1}\right) \ldots \sigma\left(A_{n}\right)$, where $z_{i}$ is a fresh variable of type $\sigma_{\square}\left(S_{i}\right)$. If $z_{i}$ occurs in this normal form, put $C_{i}=\sigma\left(A_{i}\right)$, $C_{i}^{\prime}=\sigma\left(A_{i}^{\prime}\right)$ and $H_{i}=\Phi\left(\left\langle\Delta, \forall y_{1}: \sigma_{\square}\left(R_{1}\right), \ldots, \forall y_{m}: \sigma_{\square}\left(R_{m}\right) ; \sigma_{\square}\left(A_{i}^{\prime}\right) ; C_{i}^{\prime}\right\rangle, \sigma\right)$. $\left(H_{i}\right.$ is defined by Lemma 5.14 and the fact that $\left.\sigma_{\square}\left(\sigma_{\square}(A)\right) \equiv \sigma_{\square}(A)\right)$. Otherwise put $C_{i}:=z_{i}$ and $H_{i}$ a tree consisting of an unlabeled node (we call $z_{i}$ a dummy variable). Let $\left\{z_{i_{1}}, \ldots, z_{i_{k}}\right\}$ be the set of new variables thus obtained. Now the tree $\Phi(P, \sigma)$ consists of a root labeled with

$\left\langle\Delta, \forall z_{i_{1}}: \sigma_{\square}\left(S_{i_{1}}\right), \ldots, \forall z_{i_{k}}: \sigma_{\square}\left(S_{i_{k}}\right) ; x C_{1} \ldots C_{n} ; B\right\rangle$ and subtrees $H_{1}, \ldots, H_{n}$ (ordered from left to right).

Compare the form of the triples in $\Phi(P, \sigma)$ with the $\forall \exists \forall$ format in [17]. Note that each label of a node in $\Phi(P, \sigma)$ is of the form $\left\langle\Delta ; x C_{1} \ldots C_{n} ; D\right\rangle$. The phrase a triple $P^{\prime}$ in $\Phi(P, \sigma)$ will mean a node $p^{\prime}$ in $\Phi(P, \sigma)$ labeled with $P^{\prime}$. The depth of $P^{\prime}$ in $\Phi(P, \sigma)$ is the number of labeled 
nodes in the path from the root to $p^{\prime}$, not counting $p^{\prime}$. The depth of $\Phi(P, \sigma)$ is the maximal depth of a triple in $\Phi(P, \sigma)$. Let $Q=\left\langle\Delta ; x C_{1} \ldots C_{n} ; D\right\rangle$ and $Q^{\prime}=\left\langle\Delta^{\prime} ; x^{\prime} C_{1}^{\prime} \ldots C_{n}^{\prime} ; D^{\prime}\right\rangle$ be triples in $\Phi(P, \sigma)$. Then we say that the head $x$ is below the head $x^{\prime}$ if $Q$ is below $Q^{\prime}$. The tree structure of $\Phi(P, \sigma)$ is only occasionally used; mostly we identify $\Phi(P, \sigma)$ with the set of its labels.

Lemma 5.16. Let $P=\langle\Gamma ; A ; B\rangle$ be a third-order matching problem for objects in $\lambda \circ$ and let $\sigma$ be a solution for $P$. Then $\Phi(P, \sigma)$ is a collection of third-order matching problems for objects in $\lambda \circ$.

Proof. By induction on the length of $\sigma_{\square}(A)$. The reasoning is similar to the reasoning in the proof of Lemma 5.14.

\section{Lemma 5.17.}

1. Let $P$ be an third-order matching problem in $\lambda \circ$ and $\sigma$ a solution for $P$. Then $\sigma$ is a solution for $\Phi(P, \sigma)$.

2. Let $P$ be a third-order matching problem in $\lambda \tau$ and $\sigma$ a solution for $P$. If $\tau$ is a solution for $\Phi(P, \sigma)$, then $\tau$ is a solution for $P$.

Proof. cf. [8].

Definition 5.18. Let $P=\langle\Gamma ; A ; B\rangle$ be a third-order matching problem for objects in $\lambda \underline{\omega}$ and let $\sigma$ be a solution for $P$. Define $|\Phi(P, \sigma)|$ as the underlying tree of $\Phi(P, \sigma)$ but with each label $\langle\Delta ; C ; D\rangle$ replaced by $\langle|\Delta| ;|C| ;|D|\rangle$. We call $\langle\Delta ; C ; D\rangle$ and $\langle|\Delta| ;|C| ;|D|\rangle$ corresponding. Note that the use of the $\|$-function is justified by Lemma 5.16 .

Lemma 5.19. Let $P=\langle\Gamma ; A ; B\rangle$ be a third-order matching problem in $\lambda \underline{\omega}$ and let $\sigma$ be a solution for $P, \operatorname{dom}(\sigma) \subseteq \operatorname{dom}(\Gamma)$. Then for a suitable choice of variables we have $|\Phi(P, \sigma)|=\Phi(|P|,|\sigma|)$.

Proof. By induction on the length of $\sigma_{\square}(A)$, using the results of Subsection 5.1.

The phrase "suitable" means the following. When we shift in the construction of $\Phi(P, \sigma)$ from $\Phi(\langle\Gamma ; \lambda x: A . B ; \lambda x: A . C\rangle, \sigma)$ to $\Phi(\langle\Gamma, \forall x: A ; B ; C\rangle, \sigma)$ and we shift in the construction of $\Phi(|P|,|\sigma|)$ from $\Phi(\langle|\Gamma| ; \lambda y:|A| .|B| ; \lambda y:|A| .|C|\rangle,|\sigma|)$ to $\Phi(\langle|\Gamma|, \forall y:|A| ;|B| ;|C|\rangle,|\sigma|)$ at the same place in the underlying tree, then the variable $x$ and $y$ should be identical. Likewise, dummy variables added at corresponding places should be chosen identical. Below we will assume that the choice is made "suitably".

If $P=\langle\Gamma ; A ; B\rangle$ is a third-order matching problem for objects in $\lambda \underline{\omega}$ and we (only) have a solution $\tau$ for $|P|$ we cannot speak of correspondence in the sense of the previous definition. Still, we need to be and are able to relate matching problems in $\Phi(|P|, \tau)$ to extensions of $\Gamma$ and subterms of $A$. Below we develop some terminology for this goal. 
Definition 5.20. Suppose $\Gamma \vdash_{\lambda_{\circ}} A: C: *$. By induction on the LNF-structure of $A$ we define a tree, $\operatorname{witn}(\Gamma, A)$ (witn for witness). The nodes of witn $(\Gamma, A)$ are labeled with pairs of contexts and subterms of $A$. Write $A \equiv \lambda x_{1}: S_{1} \ldots \lambda x_{n}: S_{n} . x A_{1} \ldots A_{m}$. Then witn $(\Gamma, A)$ consists of a root labeled with the pair consisting of $\Gamma^{\prime} \equiv \Gamma, \forall x_{1}: S_{1}, \ldots, \forall x_{n}: S_{n}$ and $x A_{1} \ldots A_{m}$ and subtrees $\operatorname{witn}\left(\Gamma^{\prime}, A_{1}\right), \ldots, \operatorname{witn}\left(\Gamma^{\prime}, A_{m}\right)$ (ordered from left to right).

Lemma 5.21. Suppose $\Gamma \vdash_{\lambda \circ} A: C: *$. Let the pair $\Delta, D$ be the label of a node in witn $(\Gamma, A)$. Then $\Delta \vdash_{\lambda \circ} D: E$ for some term $E$. Moreover $\Delta=_{\square} \Gamma$.

Proof. By induction on the LNF-structure of $A$.

Let $P=\langle\Gamma ; A ; B\rangle$ be a third-order matching problem for objects in $\lambda \circ$ and $\sigma$ a solution for $P$. Write $T_{1}$ for the underlying tree of $\operatorname{witn}(\Gamma, A)$ and write $T_{2}$ for the underlying tree of $\Phi(P, \sigma)$. Then it easy to see that $T_{2}$ can be viewed as the result of replacing some subtrees in $T_{1}$ by leaves. Thus every path starting from the root in $T_{2}$ corresponds to a path starting from the root of $T_{1}$. This gives a correspondence between triples in $\Phi(P, \sigma)$ and nodes in $T_{1}$. Let $\Delta^{\prime}, C^{\prime}$ be the label of the node in $T_{1}$ corresponding to a triple $P^{\prime}=\langle\Delta ; C ; D\rangle$ in $\Phi(P, \sigma)$. The pair $\Delta^{\prime}, C^{\prime}$ is called the witnessing pair for $P^{\prime} ; \Delta^{\prime}$ is called the witnessing context for $P^{\prime}$ and $C^{\prime}$ the witnessing term for $P^{\prime}$.

Lemma 5.22. Let $P=\langle\Gamma ; A ; B\rangle$ be a third-order matching problem for objects in $\lambda \circ$. Let $P^{\prime}=\langle\Delta ; C ; D\rangle$ be a triple in $\Phi(P, \sigma)$. We can write $\Delta \equiv \Delta_{1}, \Delta_{2}$, where $\Delta_{2}$ consists of dummy variables added in the construction of $\Phi(P, \sigma)$. Let $\Delta^{\prime}, C^{\prime}$ be the witnessing pair for $P^{\prime}$. Then $\sigma$ is well-typed in $\Delta^{\prime}$ and $\sigma\left(C^{\prime}\right)={ }_{\beta \eta} D$. If $\lambda \circ$ is $\lambda \tau$ then $\Delta_{1} \equiv \Delta^{\prime}$; if $\lambda \circ$ is $\lambda \underline{\omega}$ then $\Delta_{1} \equiv \sigma_{\square}\left(\Delta^{\prime}\right)$.

Proof. By inspection of the definition of $\Phi(P, \sigma)$ and witn $(\Gamma, A)$.

Lemma 5.23. Let $P=\langle\Gamma ; A ; B\rangle$ be a third-order matching problem for objects in $\lambda \underline{\omega}$. Let $\sigma$ be a solution for $P$. Let $\langle\Delta ; C ; D\rangle$ in $\Phi(P, \sigma)$ and $\langle|\Delta| ;|C| ;|D|\rangle$ in $\Phi(|P|,|\sigma|)$ be corresponding. If $\Delta^{\prime}, C^{\prime}$ is the witnessing pair for $\langle\Delta ; C ; D\rangle$ in $\Phi(P, \sigma)$, then $\left|\Delta^{\prime}\right|,\left|C^{\prime}\right|$ is the witnessing pair for $\langle|\Delta| ;|C| ;|D|\rangle$ in $\Phi(|P|,|\sigma|)$.

Proof. By induction on the length of $\sigma_{\square}(A)$.

Let $P=\langle\Gamma ; A ; B\rangle$ be a third-order matching problem for objects in $\lambda \underline{\omega}$. Write $T_{1}$ for the underlying tree of $\operatorname{witn}(\Gamma, A)$. When we write $T_{2}$ for the underlying tree of $\operatorname{witn}(|\Gamma|,|A|)$ then we see that $T_{2}$ equals $T_{1}$. Let $\tau$ be a solution for $|P|$. Let $T_{3}$ be the underlying tree of $\Phi(|P|, \tau)$. Since, as we have seen above, each node in $T_{3}$ corresponds to a node in $T_{2}$, each such node also corresponds to a node in $T_{1}$. Now for each triple $\langle\Delta ; C ; D\rangle$ in $\Phi(|P|, \tau)$ we define the $\lambda \underline{\omega}$-companion to $\Delta$ as the context part of the label of the corresponding node in $T_{1}$.

Lemma 5.24. Let $P=\langle\Gamma ; A ; B\rangle$ be a third-order matching problem for objects in $\lambda \underline{\omega}$. Suppose $\sigma$ is a solution for $|P|, \operatorname{dom}(\sigma) \subseteq \operatorname{dom}(|\Gamma|)$. Let $Q=\langle\Delta ; C ; D\rangle$ be a triple in $\Phi(|P|, \sigma)$ and let $\Xi$ be the $\lambda \underline{\omega}$-companion to $\Delta$. Then $|\Xi| \subseteq \Delta$. If $x$ occurs free in $D$ and is of non-atomic type in $\Delta$ then $x$ is universal in $\Xi$ and of closed type in $\Xi$.

Proof. By inspection of the definition of $\Phi(|P|, \sigma)$ and witn $(\Gamma, A) . \quad \square$ 
As mentioned before, Dowek used the sets defined above to transform solutions into more efficient solutions. By slightly changing this transformation, these efficient solutions may be assumed to have a certain desirable "standard" form. We will describe this form in several steps.

Definition 5.25. Let $\Gamma$ be a context legal in $\lambda \circ$. We say that a pseudo-term $t$ is pre-well-typed in $\Gamma$ if

- for some term $S$ we have $|\Gamma| \vdash_{\lambda \tau}|t|: S$ and $|t|$ is in LNF (w.r.t. $|\Gamma|$ ).

- For every domain $D$ in $t$ which is not inside another domain in $t$ we have $\Gamma \vdash_{\lambda \circ} D: *$.

- For every variable $y \in F V(t)$, not occurring in a domain in $t, \Gamma(y)$ is defined and $\Gamma \vdash_{\lambda \circ} \Gamma(y): *$.

For example in the context $\left\langle\forall B: *, \forall X: * \rightarrow *, \exists A_{1}: *, \forall g: X B \rightarrow X B \rightarrow X B, \exists x: X B\right\rangle$, which is legal in $\lambda \underline{\omega}$, the term $\lambda y: X A_{1} . g x y$ is pre-well-typed but not typable. The term becomes typable when we substitute $B$ for $A_{1}$.

If $\lambda x_{1}: S_{1} \ldots \lambda x_{n}: S_{n} . y t_{1} \ldots t_{m}$ is pre-well-typed in $\Gamma$ then $\lambda x_{2}: S_{2} \ldots \lambda x_{n}: S_{n} . y t_{1} \ldots t_{m}$ is prewell-typed in $\Gamma, \forall x_{1}: S_{1}$ and, for all $1 \leq i \leq m, t_{i}$ is pre-well-typed in $\Gamma, \forall x_{1}: S_{1}, \ldots, \forall x_{n}: S_{n}$.

The following definition is perhaps a bit hard to swallow. Therefore we first give an informal version. A substitution $\sigma$ will be called standard in $\Gamma$ if for all substitution terms $t$ the following holds. All free object variables are either universal and their types are closed (so they are "constants"), or they are existential, but then they function only as dummy arguments: they are not themselves applied to arguments. So their type is atomic (but not necessarily closed). Furthermore we want each such existential variable to have a unique occurrence in $\sigma$. If moreover $\sigma$ is a solution for $P=\langle\Gamma ; A ; B\rangle$ then we call $\sigma$ a standard solution for $P$ if every universal variable, free in $t$, occurs in $B$ (otherwise this variable only occurs in dummy arguments which should be replaced by existential variables). In this way, these parts of substitution terms that could be replaced by other terms are of a very simple form. This is essential for us, because, precisely by the "replaceability" of such parts, reasoning about them is in general very hard. (The last clause in the definition below is added for technical reasons.)

Definition 5.26. Let $\Gamma$ be legal in $\lambda \circ$. Let $t=\lambda x_{1}: S_{1} \ldots \lambda x_{n}: S_{n} . y t_{1} \ldots t_{m}$ be pre-well-typed in $\Gamma$. Let $Z$ be a set of variables in $\Gamma$. Then $t$ is called standard in $\Gamma$ and $Z$ if for every $x \in F V(t)$ such that $x \notin Z$ and $\Gamma(x) \notin \mathcal{K}_{\square}$ we have that either $x$ is universal in $\Gamma$ and $\Gamma(x)$ is closed in $\Gamma$, or $x$ is existential in $\Gamma, \Gamma(x)$ is atomic and $x$ has a unique occurrence in $t$.

We extend the definition to substitutions. Suppose $\operatorname{dom}(\sigma) \subseteq \operatorname{dom}(\Gamma)$. Then we call $\sigma$ standard in $\Gamma$ if, first, for all triples $\left\langle x ; \gamma ; \lambda x_{1}: S_{1} \ldots \lambda x_{n}: S_{n} . y t_{1} \ldots t_{m}\right\rangle$ in $\sigma$ such that $\Gamma(x) \notin$ $\mathcal{K}_{\square}, y t_{1} \ldots t_{m}$ is standard in $\sigma\left(\Gamma_{x}\right), \gamma, \forall x_{1}: S_{1}, \ldots, \forall x_{n}: S_{n}$ and $\left\{x_{1}, \ldots, x_{n}\right\}$. Moreover we demand that for each such triple $\langle x ; \gamma ; t\rangle$ in $\sigma$, we have that $\gamma$ consists of declarations of the form $\exists z: C$ with $C$ atomic and $C \notin \mathcal{K}_{\square}$ and $\operatorname{dom}(\gamma) \subseteq F V(t)$. Conversely, if $x \in F V(t)$ is existential in $\sigma\left(\Gamma_{x}\right), \gamma$ and its type is not in $\mathcal{K}_{\square}$, then $x \in \operatorname{dom}(\gamma)$.

Finally, let $P=\langle\Gamma ; A ; B\rangle$ be a matching problem for objects in $\lambda \circ$ and suppose $\sigma$ is a solution for $P$. Then $\sigma$ is called a standard solution for $P$ if $\sigma$ is standard in $\Gamma$ and 
moreover every triple $\left\langle x ; \gamma ; \lambda x_{1}: S_{1} \ldots \lambda x_{n}: S_{n} . y t_{1} \ldots t_{m}\right\rangle \in \sigma$ such that $\Gamma(x) \notin \mathcal{K}_{\square}$ satisfies the following two requirements. For all $z$ in $F V\left(\lambda x_{1}: S_{1} \ldots \lambda x_{n}: S_{n} . y t_{1} \ldots t_{m}\right)$ such that $z$ is universal in $\sigma\left(\Gamma_{x}\right)$ and $\Gamma(z) \notin \mathcal{K}_{\square}$ we have $z \in F V(B)$. For every subterm of the form $x_{i} s_{1} \ldots s_{l}$, where $x_{i}(1 \leq i \leq n)$ is of type $S_{i} \equiv R_{1} \rightarrow \cdots \rightarrow R_{l} \rightarrow R$ and for every $1 \leq j \leq l$ we have that either there is some triple $\left\langle\Delta ; x C_{1} \ldots C_{n} ; D\right\rangle$ in $\Phi(P, \sigma)$ such that $C_{i}$ is relevant in its $j^{\text {th }}$ argument and is not a dummy variable or $s_{j}$ is of the form $\lambda y_{1}: \sigma\left(R_{1}\right) \ldots \lambda y_{l}: \sigma\left(R_{l}\right) . e$ where $e$ is of atomic type $\sigma(R)$ in $\gamma$.

If $P$ is a matching problem for types in $\lambda \underline{\omega}$ then a standard solution for $P$ is defined as a $\square$-substitution satisfying the criteria for a standard solution given above but with clauses demanding pre-well-typedness, ' $\Gamma(z) \notin \mathcal{K}_{\square}$ ', ' $\Gamma(x) \notin \mathcal{K}_{\square}$ ' removed from each of the three stages.

Lemma 5.27. Suppose $\Gamma$ is legal in $\lambda \underline{\omega}$ and $\lambda x_{1}: S_{1} \ldots \lambda x_{n}: S_{n} . y t_{1} \ldots t_{m}$ is standard in $\Gamma$ and $Z$. Suppose that for $1 \leq i \leq n, S_{i}$ is closed in $\Gamma$. Then $t_{i}$ is standard in $\Gamma, \forall x_{1}: S_{1}, \ldots, \forall x_{n}$ : $S_{n}$.

Proof. Immediate.

Theorem 5.28 (Dowek). Let $P=\langle\Gamma ; A ; B\rangle$ be a third-order matching problem in $\lambda \tau$ or a third-order matching problem for types in $\lambda \underline{\omega}$. From a solution $\sigma$ for $P$ one can construct a substitution $\sigma^{\star}$ for $P$ such that

1. $\sigma^{\star}$ is a standard solution for $P$;

2. $x \in \operatorname{dom}\left(\sigma^{\star}\right)$ iff $x$ is a head in $\Phi(P, \sigma)$.

(Note that $\sigma^{\star}$ depends not only on $\sigma$ but also on $P$.) Moreover there exists a set, $\operatorname{Sol}(P)$, containing standard solutions for $P$, such that if $P$ has a solution $\sigma$ then $\sigma^{\star} \in \operatorname{Sol}(P)$ and such that for some $n \in \mathbb{N}$, computable from $P$ only, $\operatorname{Sol}(P)$ can be enumerated in time bounded by $n$.

Proof. By a slight modification of Dowek's proof.

Proposition 5.29. Let $P=\langle\Gamma ; A ; B\rangle$ be a third-order matching problem for objects in $\lambda \underline{\omega}$. Let $\sigma$ be a solution for $P$. The construction of $|\sigma|^{\star}$ from $|\sigma|$ w.r.t. $|P|$ can be mimicked in $\sigma$ and yields a substitution $\sigma^{\star}$ which is well-typed and standard in $\Gamma$. Moreover $\left|\sigma^{\star}\right|=|\sigma|^{\star}$. Note that from this follows by Lemma 5.13 that $\sigma^{\star}$ is also a standard solution for $P$ and (by Lemma 5.19) that $\left|\Phi\left(P, \sigma^{\star}\right)\right|=\Phi\left(|P|,|\sigma|^{\star}\right)$.

Proof. By inspection of the construction of $|\sigma|^{\star}$.

This is the main point where use is being made of new existential variables in substitution terms. Dowek's construction of an efficient solution from an arbitrary solution makes use of a variable of type $O$. From this variable, canonical terms are constructed for every type. In the context of $\lambda \underline{\omega}$ this would mean that we would have for every atomic type a variable of that 
type. But the assumption of having such a variable for each type in a given context cannot be maintained. For say we have a variable of type $X A$ with $X$ universal and $A$ existential. Then it may be the case that a solution substitutes a term for $A$ and that the construction would need another variable of the resulting type. Such a variable need not be there initially; so we need the possibility to introduce new variables.

\subsection{Lifting solutions from $\lambda \tau$ to $\lambda \underline{\omega}$}

In this section we describe a way to lift solutions for matching problems in $\lambda \tau$ to solutions for matching problems in $\lambda \underline{\omega}$. We first decorate $\lambda \tau$ terms in a naive way. This procedure need not yield terms that are well-typed in $\lambda \underline{\omega}$ (they will be pre-well-typed). In order to change them into well-typed ones, we define a third-order matching problem for types such that solutions for this matching problem, when applied to the decorated terms, yield terms of the desired type.

Definition 5.30. Suppose $\Gamma \vdash_{\lambda_{\circ}} S: *$ and $\Gamma \vdash_{\lambda_{\circ}} T: *, S$ and $T$ in HNF. Then $S$ and $T$ are called twins if $|S|_{T} \equiv|T|_{T}$. If we write $S \equiv S_{1} \rightarrow \cdots \rightarrow S_{n} \rightarrow S^{\prime}$ and $T \equiv T_{1} \rightarrow \cdots \rightarrow T_{n} \rightarrow T^{\prime}$, then the twinness of $S$ and $T$ implies the twinness of $S_{i}$ and $T_{i}(1 \leq i \leq n)$. So we can say that $S_{i}$ is the twin of $T_{i}$ in $T$.

Definition 5.31. Let $\Gamma$ be legal in $\lambda \underline{\omega}$. Suppose $\Delta \vdash_{\lambda \tau} \lambda x_{1}: S_{1} \ldots \lambda x_{n}: S_{n} . y t_{1} \ldots t_{m}: S$ and $\Gamma(z)$ and $\Delta(z)$ are twins, for all $z \in F V\left(\lambda x_{1}: S_{1} \ldots \lambda x_{n}: S_{n} . y t_{1} \ldots t_{m}\right) \cap \operatorname{dom}(\Gamma)$. (Note that $\Gamma \vdash^{\lambda \underline{\omega}} \Gamma(z): *$.) Suppose $\Gamma \vdash_{\lambda \underline{\omega}} T_{1} \rightarrow \cdots \rightarrow T_{n} \rightarrow T: *$ and that $S_{i}$ and $T_{i}$ are twins, for every $1 \leq i \leq n$. Then we define:

$\operatorname{Deco}\left(\lambda x_{1}: S_{1} \ldots \lambda x_{n}: S_{n} . y t_{1} \ldots t_{m} ; T_{1} \rightarrow \cdots \rightarrow T_{n} \rightarrow T\right)_{\Gamma}=\lambda x_{1}: T_{1} \ldots \lambda x_{n}: T_{n} . t$ and

$\operatorname{Cont}\left(\lambda x_{1}: S_{1} \ldots \lambda x_{n}: S_{n} . y t_{1} \ldots t_{m} ; T_{1} \rightarrow \cdots \rightarrow T_{n} \rightarrow T\right)_{\Gamma}=\Xi$,

where (putting $\Gamma^{\prime} \equiv \Gamma, \forall x_{1}: T_{1}, \ldots, \forall x_{n}: T_{n}$ )

- If for some $R_{1}, \ldots, R_{m}, R$ we have $Q y: R_{1} \rightarrow \cdots \rightarrow R_{m} \rightarrow R \in \Gamma^{\prime}$, then $t \equiv y s_{1} \ldots s_{m}$ and $\Xi \equiv \Xi_{1}, \ldots, \Xi_{m}$. Here $s_{i} \equiv \operatorname{Deco}\left(t_{i} ; R_{i}\right)_{\Gamma^{\prime}}$ and $\Xi_{i}=\operatorname{Cont}\left(t_{i} ; R_{i}\right)_{\Gamma^{\prime}}$, for $1 \leq i \leq m$.

- If for no $R_{1}, \ldots, R_{m}, R$ we have that $Q y: R_{1} \rightarrow \cdots \rightarrow R_{m} \rightarrow R \in \Gamma^{\prime}$, then $t \equiv y$ and $\Xi \equiv\langle\exists y: T\rangle$.

We extend Deco to substitutions as follows. Let $\Gamma$ be a context legal in $\lambda \underline{\omega}$, $\sigma$ well-typed in $|\Gamma|$ and $\operatorname{dom}(\sigma) \subseteq \operatorname{dom}(|\Gamma|)$. Let $\langle x ; \gamma ; t\rangle$ be a triple in $\sigma$. Then $\operatorname{Deco}(\langle x ; \gamma ; t\rangle)_{\Gamma}=$ $\left\langle x ; \operatorname{Cont}(t ; \Gamma(x))_{\Gamma_{x}} ; \operatorname{Deco}(t ; \Gamma(x))_{\Gamma_{x}}\right\rangle$ and $\operatorname{Deco}(\sigma)_{\Gamma}=\left\{\operatorname{Deco}(\langle x ; \gamma ; t\rangle)_{\Gamma} \mid\langle x ; \gamma ; t\rangle \in \sigma\right\}$.

We give an example. Let $\Gamma$ be $\left\langle\forall B: *, \forall X: * \rightarrow *, \exists A_{1}: *, \exists A_{2}: *, \forall g: X B \rightarrow X B \rightarrow X B, \exists f:\right.$ $\left.X A_{1} \rightarrow X A_{2}\right\rangle$. Then $\Gamma$ is legal in $\lambda \underline{\omega}$. Let $\sigma$ be $\left\{\langle f ;\langle\exists x: O\rangle ; \lambda y: O . g x y\rangle\right.$. Then Deco $(\sigma)_{\Gamma}=$ $\left\{\left\langle f ;\langle\exists x: X B\rangle ; \lambda y: X A_{1} . g x y\right\rangle\right\}$. Note that $\lambda y: X A_{1} . g x y$ is not typable in the context $\langle\forall B$ : $\left.*, \forall X: * \rightarrow *, \exists A_{1}: *, \exists A_{2}: *, \forall g: X B \rightarrow X B \rightarrow X B, \exists x: X B\right\rangle$, but it is pre-well-typed in this context. The term becomes typable when we substitute $B$ for $A_{1}$. Compare this to the example below Definition 5.25. Observe that $\left\{\left\langle A_{1} ;\langle\rangle ; B\right\rangle,\left\langle A_{2} ;\langle\rangle ; B\right\rangle\right\} \circ \operatorname{Deco}(\sigma)_{\Gamma}$ is a substitution that is well-typed in $\Gamma$.

We list a number of elementary properties of Deco. 
Lemma 5.32. Let $\Gamma$ be legal in $\lambda \underline{\omega}$. Suppose $\Delta \vdash_{\lambda \tau} \lambda x_{1}: S_{1} \ldots \lambda x_{n}: S_{n} . y t_{1} \ldots t_{m}: S$ and $\Gamma(z)$ and $\Delta(z)$ are twins, for all $z \in F V\left(\lambda x_{1}: S_{1} \ldots \lambda x_{n}: S_{n} . y t_{1} \ldots t_{m}\right) \cap \operatorname{dom}(\Gamma)$. Suppose $\Gamma \vdash_{\lambda \underline{\omega}} T_{1} \rightarrow \cdots \rightarrow T_{n} \rightarrow T^{\prime}: *$ and that $S_{i}$ and $T_{i}$ are twins, for every $1 \leq i \leq n$. Write $T \equiv \bar{T}_{1} \rightarrow \cdots \rightarrow T_{n} \rightarrow T^{\prime}$ and $t \equiv \lambda x_{1}: S_{1} \ldots \lambda x_{n}: S_{n} . y t_{1} \ldots t_{m}$. Now we have:

1. If every $x \in F V(t) \backslash \operatorname{dom}(\Gamma)$ has a unique occurrence in $t$ then $\operatorname{Deco}(t ; T)_{\Gamma}$ is pre-welltyped in $\Gamma, \operatorname{Cont}(t ; T)_{\Gamma}$. Every variable in $\operatorname{Cont}(t ; T)_{\Gamma}$ has an atomic type.

2. Let $Z \subseteq \operatorname{dom}(\Gamma)$. If moreover for all $x \in F V(t) \cap \operatorname{dom}(\Gamma), x \notin Z$, we have that $x$ is universal in $\Gamma$ and $\Gamma(x)$ is closed in $\Gamma_{x}$ or $x$ is existential in $\Gamma, \Gamma(x)$ is atomic and $x$ has a unique occurrence in $t$, then $\operatorname{Deco}(t ; T)_{\Gamma}$ is standard in $\Gamma, \operatorname{Cont}(t ; T)_{\Gamma}$ and $Z$.

3. If furthermore $T$ is of finite order (w.r.t. $\Gamma$ ) and $\Gamma(z)$ is of finite order (w.r.t. $\Gamma$ ) for all $z \in Z$, then every domain in $\operatorname{Deco}(t ; T)_{\Gamma}$ is of finite order and every variable in $\operatorname{Cont}(t ; T)_{\Gamma}$ has a type of order 1 (w.r.t. every legal $\left.\Xi(\Gamma \subseteq \square \Xi)\right)$.

Proof. By induction on the LNF-structure of $t$.

Lemma 5.33. Let $P=\langle\Gamma ; A ; B\rangle$ be a third-order matching problem for objects in $\lambda \underline{\omega}, \sigma$ a standard solution for $|P|$. Let $\Delta$ be a legal extension of $\Gamma$ such that all existential variables in $\Delta$ are of order at most $n$.

1. Deco $(\sigma)_{\Gamma}(\Delta)$ is legal in $\lambda \underline{\omega}$ and $\operatorname{Deco}(\sigma)_{\Gamma}(\Delta)=_{\square} \Delta$. Moreover every existential variable in $\operatorname{Deco}(\sigma)_{\Gamma}(\Delta)$ is of order at most $n$.

2. $\operatorname{Deco}(\sigma)_{\Gamma}$ is standard in $\Gamma$. Furthermore $\left|\operatorname{Deco}(\sigma)_{\Gamma}\right|=\sigma$ (up to the order of declarations in the substitution contexts).

3. Let $\tau$ be a $\square$-substitution, well-typed in $\Delta$. Then $\tau$ is well-typed in $\operatorname{Deco}(\sigma)_{\Gamma}(\Delta)$.

Proof. (1), (2): by Lemma 5.32. For (2) note that since $\sigma$ is a standard solution for $|P|$, every universal variable $y$, free in $\sigma(x),(x$ bound by $\sigma)$ also occurs free in $|B|$ and hence in $B$. So $\Gamma(y)$ is closed in $\Gamma$. (3): by induction on the length of $\Gamma$, using Strengthening and Thinning.

Lemma 5.34. Let $P=\langle\Gamma ; A ; B\rangle$ be a third-order matching problem for objects in $\lambda \underline{\omega}, \sigma$ a standard solution for $|P|$. Let $\tau$ be a $\square$-substitution, well-typed in $\Gamma$. Then $\left|\tau \circ \operatorname{Deco}(\sigma)_{\Gamma}\right|=\sigma$ (up to a permutation of declarations in the substitution contexts).

Proof. By Lemma 5.33 (2), Lemma 5.7 (2) and Lemma 5.32 (3).

Lemma 5.35. Suppose $\Gamma \vdash_{\underline{\lambda} \underline{\omega}} t: S: *$. Then $\operatorname{Deco}(|t| ; S)_{\Gamma} \equiv t$.

Proof. By induction on the LNF-structure of $t$. 
Lemma 5.36. Suppose $\Gamma \vdash_{\lambda \underline{\omega}} T_{1} \rightarrow \cdots \rightarrow T_{n} \rightarrow T: *$ and $\Delta \vdash_{\lambda \tau} \lambda x_{1}: S_{1} \ldots \lambda x_{n}: S_{n} . y t_{1} \ldots t_{m}$ : S. Write $t \equiv \lambda x_{1}: S_{1} \ldots \lambda x_{n}: S_{n} . y t_{1} \ldots t_{m}$ and $T^{\prime} \equiv T_{1} \rightarrow \cdots \rightarrow T_{n} \rightarrow T$. Suppose that $\Gamma(y)$ and $\Delta(y)$ are twins, for all $y \in F V(t) \cap \operatorname{dom}(\Gamma)$ and that $S_{i}$ and $T_{i}$ are twins, for every $1 \leq i \leq n$. Let $\tau$ be a $\square$-substitution, well-typed in $\Gamma$. Suppose that $T^{\prime}$ and $\Gamma(y)$ (for all $y \in F V(t) \cap \operatorname{dom}(\Gamma))$ are of finite order w.r.t. $\Gamma$. Then $\tau\left(\operatorname{Deco}\left(t ; T^{\prime}\right)_{\Gamma}\right) \equiv \operatorname{Deco}\left(t ; \tau\left(T^{\prime}\right)\right)_{\tau(\Gamma)}$ and $\tau\left(\operatorname{Cont}\left(t ; T^{\prime}\right)_{\Gamma}\right) \equiv \operatorname{Cont}\left(t ; \tau\left(T^{\prime}\right)\right)_{\tau(\Gamma)}$. Moreover Deco $\left(t ; T_{1} \rightarrow \cdots \rightarrow T_{n} \rightarrow \tau(T)\right)_{\Gamma} \equiv$ $\operatorname{Deco}\left(t ; T_{1} \rightarrow \cdots \rightarrow T_{n} \rightarrow T\right)_{\Gamma}$.

Proof. By induction on the LNF-structure of $t$.

Lemma 5.37. Write $\Xi=\Gamma_{1}, \Delta, \Gamma_{2}$. Suppose $\Xi \vdash_{\lambda \underline{\omega}} t: S: *$, where $\Delta$ contains precisely all variables $x \in F V(t)$ such that $x$ is existential in $\Xi$ and $\Xi(x) \notin \mathcal{K}_{\square}$. Suppose every variable in $\operatorname{dom}(\Delta)$ is of atomic type and each such variable has a unique occurrence in $t$. Then $\operatorname{Deco}(|t| ; S) \Xi \equiv \operatorname{Deco}(|t| ; S)_{\Gamma_{1}, \Gamma_{2}}$ and $\operatorname{Cont}(|t| ; S)_{\Xi} \equiv \operatorname{Cont}(|t| ; S)_{\Gamma_{1}, \Gamma_{2}}$ (up to the order of declarations).

Proof. By induction on the LNF-structure of $t$.

Lemma 5.38. Let $\Gamma$ be legal in $\lambda \underline{\omega}$ and $\sigma$ standard in $\Gamma$. Suppose $\sigma$ binds only variables such that their types are of finite order in $\Gamma$. Then $\sigma_{\square} \circ D e c o(|\sigma|)_{\Gamma}=\sigma$ (up to the order of declarations in the substitution contexts).

Proof. It suffices to prove that for all $\langle x ; \gamma ; t\rangle \in \sigma_{*}$ we have $t \equiv \sigma_{\square}\left(\operatorname{Deco}(|t| ; \Gamma(x))_{\Gamma_{x}}\right)$ and $\gamma \equiv \sigma_{\square}\left(\operatorname{Cont}(|t| ; \Gamma(x))_{\Gamma_{x}}\right.$ ) (up to the order of declarations). We prove the first point; the second point is completely similar. Let $\langle x ; \gamma ; t\rangle \in \sigma_{*}$ be given. Then $\langle x ;|\gamma| ;|t|\rangle \in|\sigma|$. By Lemma $3.10(3), \sigma_{\square}$ is well-typed in $\Gamma$. Thus we have:

$\sigma_{\square}\left(\operatorname{Deco}(|t| ; \Gamma(x))_{\Gamma_{x}}\right) \stackrel{5.36}{\equiv} \operatorname{Deco}\left(|t| ; \sigma_{\square}(\Gamma(x))\right)_{\sigma_{\square}\left(\Gamma_{x}\right)} \stackrel{5.37}{\equiv} \operatorname{Deco}\left(|t| ; \sigma_{\square}(\Gamma(x))\right)_{\sigma_{\square}\left(\Gamma_{x}\right), \gamma} \stackrel{5.35}{\equiv} t$. That the lemmas used in the last line are indeed applicable follows easily from standardness of $\sigma$.

Next, we define a function, Targets, which given a pre-well-typed term $t$, a type $T$ and a variable $x$ of some unspecified but atomic type, returns the set of types that $x$ must have if $t$ is to be of type $T$. Of course, we eventually want this set to be a singleton, but it is convenient not to demand that at this moment. The Targets function is used in Definition 5.43.

Definition 5.39. Let $\Gamma$ be a context legal in $\lambda \underline{\omega}$ and $t \equiv \lambda x_{1}: S_{1} \ldots \lambda x_{n}: S_{n} . y t_{1} \ldots t_{m}$ be pre-well-typed in $\Gamma$. Suppose that $\Gamma \vdash_{\lambda \underline{\omega}} T_{1} \rightarrow \cdots \rightarrow T_{n} \rightarrow T: *$, where for each $i, 1 \leq i \leq n$, $S_{i}$ and $T_{i}$ are twins. Let $x \in F V(t)$ be of atomic type in $\Gamma, \Gamma(x) \notin \mathcal{K}_{\square}$. Put $I=\{i \mid x \in$ $\left.F V\left(t_{i}\right), 1 \leq i \leq n\right\}$ and $\Gamma^{\prime}=\Gamma, \forall x_{1}: S_{1}, \ldots, \forall x_{n}: S_{n}$. Note that by, pre-well-typedness, either $y$ is one of the $x_{i}$ and $S_{i} \equiv R_{1} \rightarrow \cdots \rightarrow R_{m} \rightarrow R$ (for some $R_{1}, \ldots, R_{m}, R$ ), or $\Gamma$ contains a declaration $Q y: R_{1} \rightarrow \cdots \rightarrow R_{m} \rightarrow R$. Thus we can define

$$
\begin{aligned}
& \text { Targets }\left(x ; \lambda x_{1}: S_{1} \ldots \lambda x_{n}: S_{n} . y t_{1} \ldots t_{m} ; T_{1} \rightarrow \cdots \rightarrow T_{n} \rightarrow T\right)_{\Gamma}= \\
& \begin{cases}\{T\} & \text { if } y \equiv x \\
\bigcup_{i \in I} \operatorname{Targets}\left(x ; t_{i} ; R_{i}\right)_{\Gamma^{\prime}} & \text { otherwise }\end{cases}
\end{aligned}
$$


It follows from pre-well-typedness that $\operatorname{Targets}\left(x ; t_{i} ; R_{i}\right)_{\Gamma^{\prime}}$ is defined.

Lemma 5.40. Suppose $\Gamma \vdash_{\lambda \underline{\omega}} t: S: *, x \in F V(t), \Gamma(x)$ atomic, $\Gamma(x) \notin \mathcal{K}_{\square}$. Then Targets $(x ; t ; S)_{\Gamma}=\{\Gamma(x)\}$.

Proof. By induction on the LNF-structure of $t$.

The next lemma states that in a crucial case $\operatorname{Targets}\left(x ; t ; T_{1} \rightarrow \cdots \rightarrow T_{n} \rightarrow T\right)_{\Gamma}$ contains only closed types.

Lemma 5.41. Let $\Gamma$ be legal in $\lambda \underline{\omega}$ and suppose $\Delta \vdash_{\lambda \tau} \lambda x_{1}: S_{1} \ldots \lambda x_{n}: S_{n} . y t_{1} \ldots t_{m}: S$. Write $t \equiv \lambda x_{1}: S_{1} \ldots \lambda x_{n}: S_{n} . y t_{1} \ldots t_{m}$. Suppose that for all $z \in F V(t)$ we have that $z$ is universal in $\Gamma, \Gamma(z)$ is a twin of $\Delta(z)$ and $\Gamma(z)$ is atomic or closed in $\Gamma$. Write $T^{\prime} \equiv T_{1} \rightarrow \cdots \rightarrow T_{n} \rightarrow T$. Suppose $\Gamma \vdash_{\lambda \underline{\omega}} T^{\prime}: *$, that $T^{\prime}$ is closed in $\Gamma$ and that for all $1 \leq i \leq n, S_{i}$ and $T_{i}$ are twins. (Note that by Lemma 5.32, Deco $\left(t ; T^{\prime}\right)_{\Gamma}$ is pre-well-typed in $\Gamma$ ). Finally, let $x \in$ $F V\left(\operatorname{Deco}\left(t ; T^{\prime}\right)_{\Gamma}\right)$ be of atomic type in $\Gamma, \Gamma(x) \notin \mathcal{K}_{\square}$. Then we have:

1. For all $U$ in Targets $\left(x ; \operatorname{Deco}\left(t ; T^{\prime}\right)_{\Gamma} ; T^{\prime}\right)_{\Gamma}$ we have that $\Gamma \vdash_{\lambda \underline{\omega}} U: *, U$ is closed and atomic in $\Gamma$.

2. If moreover $\Delta$ satisfies the same properties w.r.t. $t$ as $\Gamma$ and for all $z$, free in $t$ and universal in $\Gamma$ such that $\Gamma(z)$ is not atomic we have $\Gamma(z) \equiv \Delta(z)$, then

$\operatorname{Targets}\left(x ; \operatorname{Deco}\left(t ; T^{\prime}\right)_{\Gamma} ; T^{\prime}\right)_{\Gamma}=\operatorname{Targets}\left(x ; \operatorname{Deco}\left(t ; T^{\prime}\right)_{\Gamma} ; T^{\prime}\right)_{\Delta}$.

Proof. By induction on the LNF-structure of $t$.

Write $\operatorname{Deco}\left(t ; T^{\prime}\right)_{\Gamma} \equiv \lambda x_{1}: T_{1} \ldots \lambda x_{n}: T_{n} . y t_{1}^{\prime} \ldots t_{m}^{\prime}$. If $x \equiv y$, then the result is obviously true. If not then for some $i, 1 \leq i \leq m, x \in F V\left(t_{i}^{\prime}\right)$. Now either $y \equiv x_{j}$, for some $j, 1 \leq j \leq n$, where $T_{j} \equiv R_{1} \rightarrow \cdots \rightarrow R_{m} \rightarrow R$ or a declaration $\forall y: R_{1} \rightarrow \cdots \rightarrow R_{m} \rightarrow R(m \geq 1)$ appears in $\Gamma$ (hence in $\Delta$ ). In both cases $R_{k}(1 \leq k \leq m$ ) is closed in $\Gamma$ (resp. $\Delta$ ) and hence in $\Gamma^{\prime}$, where $\Gamma^{\prime} \equiv \Gamma, \forall x_{1}: T_{1} \ldots \forall x_{n}: T_{n}$ (resp. $\Delta^{\prime} \equiv \Delta, \forall x_{1}: T_{1} \ldots \forall x_{n}: T_{n}$ ). Hence by induction hypothesis every $U$ in Targets $\left(x ; t_{i}^{\prime} ; R_{i}\right)_{\Gamma^{\prime}}$ is closed and atomic in $\Gamma^{\prime}$ and (since $\forall k, 1 \leq k \leq n$, $\left.x_{k} \notin F V\left(R_{i}\right)\right)$ closed and atomic in $\Gamma$.

Moreover Targets $\left(x ; t_{i}^{\prime} ; R_{i}\right)_{\Gamma^{\prime}}=\operatorname{Targets}\left(x ; t_{i}^{\prime} ; R_{i}\right)_{\Delta^{\prime}}$.

$\nabla$

Next we will sketch the matching problem we need for the proof of decidability of third-order matching for objects in $\lambda \underline{\omega}$; this sketch is made precise in Definition 5.43. First we define an auxiliary notion.

Definition 5.42. Let $t \equiv \lambda x_{1}: S_{1} \ldots \lambda x_{n}: S_{n} . y t_{1} \ldots t_{m}$ be legal in $\lambda \circ$. Let $1 \leq i \leq n$. We call $t$ relevant in its $i^{\text {th }}$ argument if $x_{i} \in F V\left(y t_{1} \ldots t_{m}\right)$.

Let $P=\langle\Gamma ; A ; B\rangle$ be a matching problem for objects in $\lambda \underline{\omega}$ and $\sigma$ a standard solution for $|P|$. Let $x$ be existential in $\Gamma$ and suppose $x$ is bound by $\sigma$. Let $t \equiv \lambda x_{1}: S_{1} \ldots \lambda x_{n}: S_{n} . y t_{1} \ldots t_{m}$ be a subterm of the decorated version of $\sigma(x)$ in $\Gamma$ (w.r.t. some type) and $T \equiv T_{1} \rightarrow \cdots \rightarrow T_{n} \rightarrow T^{\prime}$ a type, closed in $\Gamma$. We assume that $t$ is standard in some extension of $\Gamma$ and $\left\{z_{1}, \ldots, z_{k}\right\}$ and 
we assume the decorated version of $\sigma(x)$ to be of the form $\lambda z_{1}: Z_{1} \ldots \lambda z_{k}: Z_{k} . s$. We want to define by induction on the length of $t$ a matching problem such that every solution $\tau$ for this problem is such that $\tau(t)$ is of type $T$. Obviously we have to match each $S_{i}$ with $T_{i}$. Now if $y$ is free in $t$ and not in $\left\{z_{1}, \ldots, z_{k}\right\}$ then, by standardness of $t$, there are two possibilities for $y$. Either $y$ is universal in $\Gamma$ and of closed type $R_{1} \rightarrow \cdots \rightarrow R_{m} \rightarrow R$. Hence we have to match $R$ with $T^{\prime}$ and by induction we know how to deal with $t_{j}$ and $R_{j}$, for all $1 \leq j \leq m$. Or $y$ is existential in $\Gamma$ and of atomic type $R$, hence $m=0$ and we are done if we can match $R$ with $T^{\prime}$. If $y$ is a bound variable $x_{i}$, then $S_{i}$ is of the form $R_{1} \rightarrow \cdots \rightarrow R_{m} \rightarrow R$ and since we have matched $S_{i}$ with $T_{i}$ (so we can write $T_{i} \equiv R_{1}^{\prime} \rightarrow \cdots \rightarrow R_{m}^{\prime} \rightarrow R^{\prime}$ ) we have by induction a matching problem for $t_{j}$ and $R_{j}^{\prime}$ and we have to match $R^{\prime}$ with $T^{\prime}$. If $y \in\left\{z_{1}, \ldots, z_{k}\right\}$, say $y \equiv z_{i}$, then it is not evident which closed type $R_{j}$ we should use as input for the matching problem for $t_{j}$ and $R_{j}$, which is by induction defined. The idea is to look at every matching problem $\left\langle\Delta ; x D_{1} \ldots D_{n} ; C\right\rangle$ in $\Phi(|P|, \sigma)$ such that $D_{i}$ is relevant in its $j^{\text {th }}$ argument and not a dummy variable. (In Remark 5.46 we discuss the case where such a matching problem does not exist.) Because $\sigma$ is a solution for $\Phi(|P|, \sigma), D_{i}$ will be substituted for $z_{i}$ and applied to $t_{1}, \ldots, t_{m}$. From $D_{i}$ we try to read off the type $t_{j}$ should have in order that $D_{i}$ can be applied to $t_{1}, \ldots, t_{m} . D_{i}$ is a term whose type is of order $\leq 2$. So either $D_{i}$ is of the form $\lambda y_{1}: O \ldots y_{m}: O . y_{j}$ and we take $R_{j} \equiv T^{\prime}$ or $D_{i}$ is of the form $\lambda y_{1}: O \ldots y_{m}: O . e s_{1} \ldots s_{l}\left(e \not \equiv y_{j}\right.$, for $1 \leq j \leq m$ ) and we obtain $R_{j}$ by the Targets function applied to $y_{j}$, the decorated version of $e s_{1} \ldots s_{l}$ and $T^{\prime}$.

Definition 5.43. Let $P=\langle\Gamma ; A ; B\rangle$ be a third-order matching problem for objects in $\lambda \underline{\omega}$ and $\sigma$ a solution for $|P|$. Let $x$ be existential in $\Gamma, \Gamma(x) \notin \mathcal{K}_{\square}$. Write $\Phi=\Phi(|P|, \sigma)$. Let $\Delta$ be legal, $\Delta=_{\square} \Gamma$. Let $t \equiv \lambda x_{1}: S_{1} \ldots \lambda x_{n}: S_{n} . y t_{1} \ldots t_{m}$ be pre-well-typed in $\Delta$. Suppose that $\Delta \vdash_{\lambda \underline{\omega}} T_{1} \rightarrow \cdots \rightarrow T_{n} \rightarrow T: *$, where $T_{i}$ is a twin of $S_{i}$, for every $1 \leq i \leq n$. Put $\Delta^{\prime}=\Delta, \forall x_{1}: T_{1}, \ldots, \forall x_{n}: T_{n}$. Let $Z$ be a set of variables that occur in $\Delta$ such that the types of the variables in $Z$ are of order at most 2 .

We define $\operatorname{SubMatch}\left(\lambda x_{1}: S_{1} \ldots \lambda x_{n}: S_{n} . y t_{1} \ldots t_{m} ; T_{1} \rightarrow \cdots \rightarrow T_{n} \rightarrow T\right)_{\Delta, Z}$. As a consequence of pre-well-typedness, $y$ is one of the $x_{i}$ and $S_{i} \equiv R_{1} \rightarrow \cdots \rightarrow R_{m} \rightarrow R$ (for some $R_{1}, \ldots, R_{m}$, $R$ ) or $\Delta$ contains a declaration $Q y: R_{1} \rightarrow \cdots \rightarrow R_{m} \rightarrow R$. We distinguish two cases.

1. $y \notin Z$. We define $\operatorname{SubMatch}\left(\lambda x_{1}: S_{1} \ldots \lambda x_{n}: S_{n} . y t_{1} \ldots t_{m} ; T_{1} \rightarrow \cdots \rightarrow T_{n} \rightarrow T\right)_{\Delta, Z}=$ $\left\{\left\langle\Gamma ; S_{i} ; T_{i}\right\rangle \mid 1 \leq i \leq n\right\} \cup\{\langle\Gamma ; R ; T\rangle\} \cup \bigcup_{1 \leq i \leq m} \operatorname{SubMatch}\left(t_{i} ; R_{i}^{\prime}\right)_{\Delta^{\prime}, Z}$.

Here $R_{i}^{\prime}$ is $R_{i}$ if $y \in \operatorname{dom}(\Delta)$ and $R_{i}^{\prime}$ is the twin of $R_{i}$ in $T_{j}$ if $y$ is $x_{j}$ (for some $1 \leq j \leq n$ ).

2. $y \in Z$, say $y \equiv z_{i}$.

Write $\Phi_{x, i, j}$ for $\left\{\left\langle\Psi ; x D_{1} \ldots D_{p} ; C\right\rangle \in \Phi \mid D_{i}\right.$ relevant in its $j^{\text {th }}$ argument, not a dummy variable $\}$. Suppose $E=\left\langle\Psi ; x D_{1} \ldots D_{p} ; C\right\rangle$ is an element of $\Phi_{x, i, j}$. Let $\Psi_{1}$ be the $\lambda \underline{\omega}$-companion to $\Psi$.

Because of typing reasons it is sufficient to distinguish the following two cases.

(a) $D_{i} \equiv \lambda y_{1}: O \ldots \lambda y_{k}: O . y_{j}$.

We define $a d d_{j, E}=\left\{\left\langle\Gamma ; R_{j} ; T\right\rangle\right\} \cup \operatorname{SubMatch}\left(t_{j} ; T\right)_{\Delta^{\prime}, Z}$. 
(b) $D_{i} \equiv \lambda y_{1}: O \ldots \lambda y_{k}: O . e s_{1} \ldots s_{l}\left(e \not \equiv y_{j}\right.$, for $\left.1 \leq j \leq k\right)$. Put $\Psi_{2}=\Psi_{1}, \forall y_{1}$ : $R_{1}, \ldots, \forall y_{k}: R_{k}$ and $\Theta=\operatorname{Targets}\left(y_{j} ; \operatorname{Deco}\left(e s_{1} \ldots s_{l} ; T\right)_{\Psi_{2}} ; T\right)_{\Psi_{2}}$. It is not difficult to check that $\Theta$ is defined. Note that because of the relevance of $D_{i}$ in its $j^{\text {th }}$ argument, $\Theta$ is non-empty. Also note that since $\Gamma=\square \Psi_{2}=\square \Delta^{\prime}$ we have $\Gamma \vdash_{\lambda \omega} U: *$ and $\Delta^{\prime} \vdash_{\lambda \omega} U: *$, for all $U \in \Theta$. We define $a d d_{j, E}=$ $\bigcup_{U \in \Theta}\left(\operatorname{SubMatch}\left(t_{j} ; U\right)_{\Delta^{\prime}, Z} \cup\left\{\left\langle\Gamma ; R_{j} ; U\right\rangle\right\}\right)$.

Now define $A d d_{j}=\bigcup_{E \in \Phi_{x, i, j}} a d d_{j, E}$. In Remark 5.46, the case where $\Phi_{x, i, j}$ is empty is discussed.

Finally, define $\operatorname{SubMatch}\left(\lambda x_{1}: S_{1} \ldots \lambda x_{n}: S_{n} . y t_{1} \ldots t_{m} ; T_{1} \rightarrow \cdots \rightarrow T_{n} \rightarrow T\right)_{\Delta, Z}=$ $\left\{\left\langle\Gamma ; S_{i} ; T_{i}\right\rangle \mid 1 \leq i \leq n\right\} \cup\{\langle\Gamma ; R ; T\rangle\} \cup \bigcup_{1 \leq j \leq m} A d d_{j}$.

Lemma 5.44. Let $P, \sigma, x, \Delta, Z, t \equiv \lambda x_{1}: S_{1} \ldots \lambda x_{n}: S_{n} . y t_{1} \ldots t_{m}$ and $T \equiv T_{1} \rightarrow \cdots \rightarrow T_{n} \rightarrow T^{\prime}$ be as in Definition 5.43. Suppose that $T_{1} \rightarrow \cdots \rightarrow T_{n} \rightarrow T^{\prime}$ is closed in $\Gamma$ and that the order of existential variables in $\Delta$ is at most 3. Suppose furthermore that $t$ is standard in $\Delta$ and $Z$. Then $\operatorname{SubMatch}(t ; T)_{\Delta, Z}$ is a finite collection of $\Gamma$-compatible third-order matching problems for types.

Proof. By induction on the length of $t$. Use Lemma 5.27 w.r.t. $\lambda x_{1}: T_{1} \ldots \lambda x_{n}: T_{n} . y t_{1} \ldots t_{m}$ to verify that the induction hypothesis is applicable (note that this term is standard in $\Gamma$ !). We treat one (crucial) detail. We have to check that in the definition $U$ is closed in $\Delta^{\prime}$, for all $U \in \operatorname{Targets}\left(y_{j} ; \operatorname{Deco}\left(e s_{1} \ldots s_{l} ; T\right)_{\Psi_{2}} ; T\right)_{\Psi_{2}}$. Since $\Delta^{\prime}=_{\square} \Psi_{2}$, it suffices to show that $U$ is closed in $\Psi_{2}$. This follows from Lemma 5.41 (1). We show that this lemma is applicable. Define $\Psi^{\prime} \equiv \Psi, \forall y_{1}: O, \ldots, \forall y_{k}: O$. Then $\Psi^{\prime} \vdash_{\lambda \tau} e s_{1} \ldots s_{l}: O$. It is easy to see that $\Psi_{2}$ is legal in $\lambda \underline{\omega}$, that $T$ is closed in $\Psi_{2}$, that $y_{j}$ is free in $e s_{1} \ldots s_{l}$ and of atomic type in $\Psi_{2}$ and that its type is not in $\mathcal{K}_{\square}$. Next we check the conditions on the free variables of $e s_{1} \ldots s_{l}$. A free variable of $e s_{1} \ldots s_{l}$ is either among $\left\{y_{1}, \ldots, y_{k}\right\}$ or not. The variables in $\left\{y_{1}, \ldots, y_{k}\right\}$ are universal in $\Psi_{2}$ and have atomic type in $\Psi_{2}$. From Lemma 2.11 we can deduce that every variable $z$ that occurs free in $e s_{1} \ldots s_{l}$ but is not among $\left\{y_{1}, \ldots, y_{k}\right\}$ occurs in $C$. (Apply this lemma with, for $A$, the normal form of $\sigma(x) D_{1} \ldots D_{i-1} z_{i} D_{i+1} \ldots D_{p}\left(z_{i}\right.$ a fresh variable of the appropriate type); $\Psi$ for $\Gamma$; $z_{i}$ for $x ;\langle\rangle$ for $\Delta ; D_{i}$ for $B$ and the normal form of $C$ for $C)$. So by Lemma 5.24, $z$ is universal in $\Psi_{1}$ and $\Gamma(z)$ is closed in $\Psi_{1}$, hence in $\Psi_{2}$. Finally, we need to show that for all $z \in F V\left(e s_{1} \ldots s_{l}\right)$ we have that $\Psi_{2}(z)$ and $\Psi^{\prime}(z)$ are twins. For the variables in $\left\{y_{1}, \ldots, y_{k}\right\}$ this is obvious. The remaining free variables are declared in $\Psi_{1}$. By Lemma 5.24 we know that $\left|\Psi_{1}\right| \subseteq \Psi$. We are done.

Next we define Match. This definition is a straightforward application of SubMatch, except that we want to use previously obtained substitutions in the definition of Match and we want to restrict the use of Submatch to heads above a fixed depth in $\Phi(|P|, \sigma)$. Hence Match gets a substitution and a natural number as extra parameters.

Definition 5.45. Let $P=\langle\Gamma ; A ; B\rangle$ be a third-order matching problem for objects in $\lambda \underline{\omega}$ and $\sigma$ a standard solution for $|P|$. Let $\rho$ be a $\square$-substitution, well-typed in $\Gamma$. Suppose the declaration $\exists x: S_{1} \rightarrow \cdots \rightarrow S_{n} \rightarrow S: *$ occurs in $\Gamma$, where $\rho(S)$ is closed in $\Gamma$ and $x$ is bound by $\sigma$ and of depth at most $d$ in $\Phi(|P|, \sigma)$. Write $S^{\prime} \equiv S_{1} \rightarrow \cdots \rightarrow S_{n} \rightarrow \rho(S)$. Let $\operatorname{Deco}\left(\sigma(x) ; S^{\prime}\right)_{\Gamma}$ be 
$\lambda x_{1}: S_{1} \ldots \lambda x_{n}: S_{n} . y t_{1} \ldots t_{m}$. Put $Z=\left\{x_{1}, \ldots, x_{n}\right\}$. Put $\Delta=\operatorname{Deco}(\sigma)_{\Gamma}(\Gamma), \forall x_{1}: S_{1}, \ldots, \forall x_{n}:$ $S_{n}$. By Lemma 5.33 (1), $\Delta$ is legal in $\lambda \underline{\omega}$ and $\Delta=\square \Gamma$. By Lemma $5.32(1), y t_{1} \ldots t_{m}$ is pre-well-typed in $\Delta$. So we can define $\operatorname{Match}(x, \rho, d)_{\Gamma}=\operatorname{SubMatch}\left(y t_{1} \ldots t_{m} ; \rho(S)\right)_{\Delta, Z} \cup$ $\{\langle\Gamma ; S ; \rho(S)\rangle\}$. Let $X$ be the set of variables $x_{i}$ of depth at most $d$ in $\Phi(|P|, \sigma)$ such that $x_{i}$ is bound by $\sigma$ and $\exists x_{i}: T_{1} \rightarrow \cdots \rightarrow T_{n_{i}} \rightarrow T_{i}$ occurs in $\Gamma$, where $\rho\left(T_{i}\right)$ is closed in $\Gamma$. Put $\operatorname{Match}(\sigma, \rho, d)_{\Gamma}=\bigcup_{x \in X} \operatorname{Match}(x, \rho, d)_{\Gamma}$.

Remark 5.46. There is one subtlety involved in the definition above. Recall that we want a solution $\tau$ of $\operatorname{Match}(x, \rho, d)_{\Gamma}$ to be such that $\tau\left(\operatorname{Deco}(\sigma)_{\Gamma}(\Gamma)\right) \vdash_{\lambda \underline{\omega}} \tau\left(\operatorname{Deco}\left(\sigma(x) ; S^{\prime}\right)_{\Gamma}\right): \tau\left(S^{\prime}\right)$. Suppose that in some recursive call of SubMatch, we are in the second case, where the head variable under consideration is an element of $Z$, say it is $z_{i}$ and $z_{i}$ is in this case the head variable of $z_{i} t_{1}^{\prime} \ldots t_{m^{\prime}}^{\prime}$. Write $S_{i}=V_{1} \rightarrow \cdots \rightarrow V_{m^{\prime}} \rightarrow V$. Suppose now that $\Phi_{x, i, j}=\emptyset$ (for some $1 \leq j \leq m^{\prime}$ ). Then the further recursive calls of Submatch in this case do not extend Match with matching problems to ensure that $\tau\left(\operatorname{Deco}(\sigma)_{\Gamma}(\Gamma)^{\prime}\right) \vdash_{\lambda \underline{\omega}} \tau\left(t_{j}^{\prime}\right): \tau\left(V_{j}\right)$, where $\operatorname{Deco}(\sigma)_{\Gamma}(\Gamma)^{\prime}$ is the current context extending $\operatorname{Deco}(\sigma)_{\Gamma}(\Gamma)$. We explain why there is no need to extend it. Write $V_{j} \equiv W_{1} \rightarrow \cdots \rightarrow W_{l} \rightarrow W$. By (the modification of) Dowek's construction of the standard term $\sigma(x), t_{j}^{\prime}$ is in this case of the form $\lambda z_{1}: W_{1} \ldots \lambda z_{k}: W_{l} . u$, where $\exists u: W$ occurs in $\operatorname{Deco}(\sigma)_{\Gamma}(\Gamma)^{\prime}$. So every substitution $\theta$, well-typed in $\operatorname{Deco}(\sigma)_{\Gamma}(\Gamma)^{\prime}$, satisfies $\theta\left(\operatorname{Deco}(\sigma)_{\Gamma}(\Gamma)^{\prime}\right) \vdash_{\lambda \underline{\omega}} \theta\left(t_{j}^{\prime}\right): \theta\left(V_{j}\right)$.

Lemma 5.47. Let $P=\langle\Gamma ; A ; B\rangle$ be a third-order matching problem for objects in $\lambda \underline{\underline{\omega}}, \sigma$ a standard solution for $|P|$ and $\rho$ a $\square$-substitution, well-typed in $\Gamma$. Let $d$ be less or equal to the depth of $\Phi(|P|, \sigma)$. Then Match $(\sigma, \rho, d)_{\Gamma}$ is a finite collection of $\Gamma$-compatible third-order matching problems for types.

Proof. We show that for each $x \in \operatorname{dom}(\sigma)$ such that $\exists x: S_{1} \rightarrow \cdots \rightarrow S_{n} \rightarrow S$ occurs in $\Gamma$ and $\rho(S)$ is closed in $\Gamma$ and the depth of $x$ in $\Phi(|P|, \sigma)$ is at most $d$, it is true that $\operatorname{Match}(x, \rho, d)_{\Gamma}$ is a finite collection of $\Gamma$-compatible third-order matching problems for types. Since $S$ is legal in $\Gamma$ and $\rho(S)$ closed in $\Gamma,\langle\Gamma ; S ; \rho(S)\rangle$ is a third-order matching problem for types in $\lambda \underline{\omega}$. Next, let $\operatorname{Deco}\left(\sigma(x) ; S_{1} \rightarrow \cdots \rightarrow S_{n} \rightarrow \rho(S)\right)_{\Gamma}$ be $t=\lambda x_{1}: S_{1} \ldots \lambda x_{n}: S_{n} . y t_{1} \ldots t_{m}$. Put $Z=\left\{x_{1}, \ldots, x_{n}\right\}$. Put $\Delta=\operatorname{Deco}(\sigma)_{\Gamma}(\Gamma), \forall x_{1}: S_{1}, \ldots, \forall x_{n}: S_{n}$. We show that SubMatch $\left(y t_{1} \ldots t_{m} ; \rho(S)\right)_{\Delta, Z}$ is a collection of $\Gamma$-compatible third-order matching problems for types in $\lambda \underline{\omega}$.

By Lemma 5.33 (1), we have that $\Delta$ is legal in $\lambda \underline{\omega}$ and $\Delta=_{\square} \Gamma$ and $\Delta$ contains only existential variables of order at most 3 . By Lemma $5.33(2)$ we have that $y t_{1} \ldots t_{m}$ is standard in $\Delta$ and $Z$. So the required fact follows from Lemma 5.44 .

Lemma 5.48. Let $P, \sigma, \rho, x, d, S^{\prime}, t \equiv \lambda x_{1}: S_{1} \ldots \lambda x_{n}: S_{n} . y t_{1} \ldots t_{m}, Z$ and $\Delta$ be as in Definition 5.45. Then for all $u \in F V\left(y t_{1} \ldots t_{m}\right) \cap\left\{x_{1}, \ldots, x_{n}\right\}$, of type $R_{1} \rightarrow \cdots \rightarrow R_{k} \rightarrow R$, there exists a type $R^{\prime}$ such that $\left\langle\Gamma ; R ; R^{\prime}\right\rangle \in \operatorname{Match}(x, \rho, d)_{\Gamma}$.

Proof. Induction on the length of $t$. Here the last clause in the definition of "standard solution" is used. 
We proceed by stating two properties of solutions $\tau$ for $\operatorname{Match}(\sigma, \rho, d)_{\Gamma}$. First, $\tau$ is well-typed in $\Gamma$ and $\operatorname{Deco}(\sigma)_{\Gamma}(\Gamma)$. Secondly, the application of $\tau$ to decorated terms originating from $\sigma$ yields terms of the desired type.

Lemma 5.49. Let $P=\langle\Gamma ; A ; B\rangle$ be a third-order matching problem for objects in $\lambda \underline{\omega}, \sigma$ a standard solution for $|P|$ and $\rho$ a $\square$-substitution, well-typed in $\Gamma$. Let $d$ be less or equal to the depth of $\Phi(|P|, \sigma)$. Suppose there exists some $x$, bound by $\sigma$, such that $\exists x: S_{1} \rightarrow \cdots \rightarrow S_{n} \rightarrow S$ occurs in $\Gamma$ and $\rho(S)$ is closed in $\Gamma$. Let $\tau$ be a solution for Match $(\sigma, \rho, d)_{\Gamma}$. Then $\tau$ is well-typed in $\Gamma$ and hence (by Lemma $5.33(3)$ ) in $\operatorname{Deco}(\sigma)_{\Gamma}(\Gamma)$.

Proof. Immediate by the definition of Match.

Lemma 5.50. Let $P=\langle\Gamma ; A ; B\rangle$ be a third-order matching problem for objects in $\lambda \underline{\omega}$, $\sigma$ a standard solution for $|P|$ and $\rho$ a $\square$-substitution, well-typed in $\Gamma$. Let $\langle x ; \gamma ; t\rangle \in \sigma$, where $\exists x: S_{1} \rightarrow \cdots \rightarrow S_{n} \rightarrow S$ occurs in $\Gamma$ and $\rho(S)$ is closed in $\Gamma$. Let $\tau$ be a solution for $\operatorname{Match}(\sigma, \rho, d)_{\Gamma}$.

Then $\tau\left(\operatorname{Deco}(\sigma)_{\Gamma}(\Gamma)\right) \vdash_{\lambda \underline{\omega}} \tau\left(\operatorname{Deco}\left(t ; S_{1} \rightarrow \cdots \rightarrow S_{n} \rightarrow S\right)_{\Gamma}\right): \tau\left(S_{1} \rightarrow \cdots \rightarrow S_{n} \rightarrow S\right)$.

Proof. Below we prove $\tau\left(\operatorname{Deco}(\sigma)_{\Gamma}(\Gamma)\right) \vdash_{\lambda \underline{\omega}} \tau\left(\operatorname{Deco}\left(t ; S_{1} \rightarrow \cdots \rightarrow S_{n} \rightarrow \rho(S)\right)_{\Gamma}\right): \tau\left(S_{1} \rightarrow \cdots \rightarrow S_{n} \rightarrow S\right)$

Since $\tau$ is by assumption a solution for $\langle\Gamma ; S ; \rho(S)\rangle$, we can write this as $\tau\left(\operatorname{Deco}(\sigma)_{\Gamma}(\Gamma)\right) \vdash_{\lambda \underline{\omega}} \tau\left(\operatorname{Deco}\left(t ; S_{1} \rightarrow \cdots \rightarrow S_{n} \rightarrow \tau(S)\right)_{\Gamma}\right): \tau\left(S_{1} \rightarrow \cdots \rightarrow S_{n} \rightarrow S\right)$.

From this follows by Lemma 5.36

$\tau\left(\operatorname{Deco}(\sigma)_{\Gamma}(\Gamma)\right) \vdash_{\lambda \underline{\omega}} \operatorname{Deco}\left(t ; \tau\left(S_{1} \rightarrow \cdots \rightarrow S_{n} \rightarrow \tau(S)\right)\right)_{\tau(\Gamma)}: \tau\left(S_{1} \rightarrow \cdots \rightarrow S_{n} \rightarrow S\right)$.

This is easily seen to be equivalent to $\left.\tau\left(\operatorname{Deco}(\sigma)_{\Gamma}(\Gamma)\right) \vdash_{\lambda \underline{\omega}} \operatorname{Deco}\left(t ; \tau\left(S_{1} \rightarrow \cdots \rightarrow S_{n} \rightarrow S\right)\right)_{\tau(\Gamma)}\right): \tau\left(S_{1} \rightarrow \cdots \rightarrow S_{n} \rightarrow S\right)$.

Using Lemma 5.36 in the reverse direction we get

$\tau\left(\operatorname{Deco}(\sigma)_{\Gamma}(\Gamma)\right) \vdash_{\lambda \underline{\omega}} \tau\left(\operatorname{Deco}\left(t ; S_{1} \rightarrow \cdots \rightarrow S_{n} \rightarrow S\right)_{\Gamma}\right): \tau\left(S_{1} \rightarrow \cdots \rightarrow S_{n} \rightarrow S\right)$.

As to $(\dagger)$. Write $\operatorname{Deco}\left(t ; S_{1} \rightarrow \cdots \rightarrow S_{n} \rightarrow \rho(S)\right)_{\Gamma} \equiv \lambda x_{1}: S_{1} \ldots \lambda x_{n}: S_{n} . y t_{1} \ldots t_{m}$. Let $Z$ be $\left\{x_{1}, \ldots, x_{n}\right\}$. Let $s$ be a subterm of $y t_{1} \ldots t_{m}$ (not a domain), such that $|s|$ is in LNF and $s$ is pre-well-typed in some extension $\Delta$ of $\operatorname{Deco}(\sigma)_{\Gamma}(\Gamma)$. Suppose that $T$ is closed in $\Delta$. Suppose furthermore that $s$ is standard in $\Delta$ and $Z$. Let $\theta$ be a solution for $\operatorname{SubMatch}(s ; T)_{\Delta, Z}$. By induction on the length of $s$ one proves: $\theta(\Delta) \vdash_{\lambda \underline{\omega}} \theta(s): T$. This induction is straightforward, when one keeps Remark 5.46 in mind. Note that since $\rho(S)$ is closed in $\Gamma, \rho(S)$ is closed in $\operatorname{Deco}(\sigma)_{\Gamma}(\Gamma)$. By taking $y t_{1} \ldots t_{m}$ for $s, \rho(S)$ for $T$ and $\operatorname{Deco}(\sigma)_{\Gamma}(\Gamma), \forall x_{1}: S_{1}, \ldots, \forall x_{n}: S_{n}$ for $\Delta$ we get:

$\tau\left(\operatorname{Deco}(\sigma)_{\Gamma}(\Gamma), \forall x_{1}: S_{1}, \ldots, \forall x_{n}: S_{n}\right) \vdash_{\lambda \underline{\omega}} \tau\left(y t_{1} \ldots t_{m}\right): \rho(S)$.

Since $\tau$ is also a solution for $\langle\Gamma ; S ; \rho(S)\rangle$, we can conclude

$\tau\left(\operatorname{Deco}(\sigma)_{\Gamma}(\Gamma)\right) \vdash_{\lambda \underline{\omega}} \tau\left(\lambda x_{1}: S_{1} \ldots \lambda x_{n}: S_{n} . y t_{1} \ldots t_{m}\right): \tau\left(S_{1} \rightarrow \cdots \rightarrow S_{n} \rightarrow S\right)$.

Corollary 5.51. Let $P=\langle\Gamma ; A ; B\rangle$ be a third-order matching problem for objects in $\lambda \underline{\omega}$, $\sigma$ a standard solution for $|P|$ and $\rho$ a $\square$-substitution, well-typed in $\Gamma$. Let $\langle x ; \gamma ; t\rangle \in \sigma$, where $\exists x: S_{1} \rightarrow \cdots \rightarrow S_{n} \rightarrow S$ occurs in $\Gamma$ and $\rho(S)$ is closed in $\Gamma$. Let $\tau$ be a solution for $\operatorname{Match}(\sigma, \rho, d)_{\Gamma}$. Put $\Delta \equiv \tau\left(\operatorname{Deco}(\sigma)_{\Gamma}\left(\Gamma_{x}\right)\right.$, Cont $\left.\left(t ; S_{1} \rightarrow \cdots \rightarrow S_{n} \rightarrow S\right)_{\Gamma_{x}}\right)$.

Then $\Delta \vdash_{\lambda \underline{\omega}} \tau\left(\operatorname{Deco}\left(t ; S_{1} \rightarrow \cdots \rightarrow S_{n} \rightarrow S\right)_{\Gamma}\right): \tau\left(S_{1} \rightarrow \cdots \rightarrow S_{n} \rightarrow S\right)$. 
Proof. We can write $\tau\left(\operatorname{Deco}(\sigma)_{\Gamma}(\Gamma)\right) \equiv \Delta, \Delta^{\prime}$, for some context $\Delta^{\prime}$. Using standardness of $\sigma$, it is easy to check that for all $z$ in $\operatorname{dom}\left(\Delta^{\prime}\right), z$ does not occur in $\tau\left(\operatorname{Deco}\left(t ; S_{1} \rightarrow \cdots \rightarrow S_{n} \rightarrow S\right)_{\Gamma}\right)$ or in $\tau\left(S_{1} \rightarrow \cdots \rightarrow S_{n} \rightarrow S\right)$. By our assumption on the form of contexts (see Lemma 2.6 (8)), we know that $z$ does not occur in a declaration $u: U$ to the right of $z$ in $\Delta^{\prime}$. So the result follows from Lemma 5.50 and Strengthening.

So $\operatorname{Match}(\sigma, \rho, d)_{\Gamma}$ does what it should do. Our next task is to check that $\operatorname{Match}\left(|\sigma|^{\star}, \rho, d\right)_{\Gamma}$ has a solution in case the initial problem $\langle\Gamma ; A ; B\rangle$ has solution $\sigma$. This is important for the completeness of the algorithm that we will use to decide whether $\langle\Gamma ; A ; B\rangle$ has a solution.

Lemma 5.52. Let $P=\langle\Gamma ; A ; B\rangle$ be a third-order matching problem for objects in $\lambda \underline{\omega}$, $\sigma$ a solution for $P$. Let $\langle x ; \gamma ; t\rangle \in|\sigma|^{\star}$, where $\exists x: S_{1} \rightarrow \cdots \rightarrow S_{n} \rightarrow S$ occurs in $\Gamma$ and $x$ is of depth at most $d$ in $\Phi\left(|P|,|\sigma|^{\star}\right)$. Suppose $\sigma_{\square}(S)$ is closed in $\Gamma$. Then $\sigma_{\square}$ is a solution for $\operatorname{Match}\left(x, \sigma_{\square}, d\right)_{\Gamma}$.

Proof. First we write the available information in a convenient way. By Proposition 5.29, $\sigma^{\star}$ is well-typed and standard in $\Gamma$. Let $\left\langle x ; \gamma^{\prime} ; t^{\prime}\right\rangle \in \sigma^{\star}$. Write $S^{\prime} \equiv S_{1} \rightarrow \cdots \rightarrow S_{n} \rightarrow S$.

Write $t \equiv \lambda x_{1}:\left|S_{1}\right| \ldots \lambda x_{n}:\left|S_{n}\right| . y t_{1} \ldots t_{m}$ and $t^{\prime} \equiv \lambda x_{1}: \sigma_{\square}\left(S_{1}\right) \ldots \lambda x_{n}: \sigma_{\square}\left(S_{n}\right) . y t_{1}^{\prime} \ldots t_{m}^{\prime}$. Then by well-typedness of $\sigma^{\star}$ we have:

$$
\sigma^{\star}(\Gamma) \vdash_{\lambda \underline{\omega}} t^{\prime}: \sigma_{\square}\left(S^{\prime}\right) .
$$

Write $\Gamma^{\prime} \equiv \sigma^{\star}(\Gamma), \forall x_{1}: \sigma_{\square}\left(S_{1}\right), \ldots, \forall x_{n}: \sigma_{\square}\left(S_{n}\right)$. Then:

$$
\Gamma^{\prime} \vdash_{\lambda \underline{\omega}} y t_{1}^{\prime} \ldots t_{m}^{\prime}: \sigma_{\square}(S) .
$$

By Lemma 5.35 we can write the previous judgement as

$$
\Gamma^{\prime} \vdash_{\lambda \underline{\omega}} \operatorname{Deco}\left(y t_{1} \ldots t_{m} ; \sigma_{\square}(S)\right)_{\Gamma^{\prime}}: \sigma_{\square}(S) .
$$

Write $\Gamma^{\prime \prime} \equiv \operatorname{Deco}\left(\left|\sigma^{\star}\right|\right)_{\Gamma}(\Gamma), \forall x_{1}: S_{1}, \ldots, \forall x_{n}: S_{n}$. Then by Lemma 5.38, we can write the previous judgement as:

$$
\sigma_{\square}\left(\Gamma^{\prime \prime}\right) \vdash_{\lambda \underline{\omega}} \operatorname{Deco}\left(y t_{1} \ldots t_{m} ; \sigma_{\square}(S)\right)_{\sigma_{\square}\left(\Gamma^{\prime \prime}\right)}: \sigma_{\square}(S) .
$$

By Lemma 5.36, this is the same as:

$$
\sigma_{\square}\left(\Gamma^{\prime \prime}\right) \vdash_{\lambda \underline{\omega}} \sigma_{\square}\left(\operatorname{Deco}\left(y t_{1} \ldots t_{m} ; S\right)_{\Gamma^{\prime \prime}}\right): \sigma_{\square}(S)
$$

That this lemma is applicable follows from Lemma 5.32 and Lemma 5.33.

Claim. Let $Z$ be $\left\{x_{1}, \ldots, x_{n}\right\}$. Let $W$ be $W_{1} \rightarrow \cdots \rightarrow W_{r} \rightarrow W^{\prime}$ be of finite order in $\Gamma$ and let $\Delta$ be a legal extension of $\operatorname{Deco}\left(|\sigma|^{\star}\right)_{\Gamma}(\Gamma)$ such that $\Gamma=_{\square} \Delta$ and $s$ a subterm of $t$ (not a domain) such that $|s|$ is in LNF and such that $\operatorname{Deco}(s ; W)_{\Delta}$ is defined and standard in $\Delta$ and $Z$. Suppose that $W_{1}, \ldots, W_{r}$ and $\sigma_{\square}\left(W^{\prime}\right)$ are closed in $\Gamma$. Finally, suppose that $\sigma_{\square}(\Delta) \vdash_{\lambda \underline{\omega}}$ $\sigma_{\square}\left(\operatorname{Deco}(s ; W)_{\Delta}\right): \sigma_{\square}(W)$. Then $\sigma_{\square}$ is a solution for $\operatorname{SubMatch}\left(\operatorname{Deco}(s ; W)_{\Delta} ; \sigma_{\square}(W)\right)_{\Delta, Z}$ 1 .

\footnotetext{
${ }^{1}$ It is not difficult to check that $\operatorname{SubMatch}\left(\operatorname{Deco}(s ; W)_{\Delta} ; \sigma_{\square}(W)\right)_{\Delta, Z}$ is defined.
} 
By taking $r=0, y t_{1} \ldots t_{m}$ for $s, S$ for $W^{\prime}$ and $\operatorname{Deco}\left(|\sigma|^{\star}\right)_{\Gamma}(\Gamma), \forall x_{1}: S_{1}, \ldots, \forall x_{n}: S_{n}$ for $\Delta$ and applying (I) we get: $\sigma_{\square}$ is a solution for $\operatorname{SubMatch}\left(\operatorname{Deco}\left(y t_{1} \ldots t_{m} ; S\right)_{\Delta} ; \sigma_{\square}(S)\right)_{\Delta, Z}$. By Lemma 5.36, we get $\sigma_{\square}$ is a solution for $\operatorname{SubMatch}\left(\operatorname{Deco}\left(y t_{1} \ldots t_{m} ; \sigma_{\square}(S)\right)_{\Delta} ; \sigma_{\square}(S)\right)_{\Delta, Z}$. It remains to show that $\sigma_{\square}$ is a solution for $\left\langle\Gamma ; S ; \sigma_{\square}(S)\right\rangle$. This is trivial.

Proof of claim. By induction on the length of $\operatorname{Deco}(s ; W)_{\Delta}$. Write $\operatorname{Deco}(s ; W)_{\Delta} \equiv$ $\lambda w_{1}: W_{1} \ldots \lambda w_{r}: W_{r} . u v_{1} \ldots v_{p}$. We distinguish two cases.

The first case is where $u \notin Z$. By standardness we know that for some $R_{1}, \ldots, R_{p}$, $R$, either $u \in \operatorname{dom}(\Delta)$ and $\Delta \vdash_{\lambda \underline{\omega}} u: R_{1} \rightarrow \cdots \rightarrow R_{p} \rightarrow R$ or $u \equiv w_{i}$ (for some $1 \leq i \leq r$ ) and $W_{i} \equiv R_{1} \rightarrow \cdots \rightarrow R_{p} \rightarrow R$. That $\sigma_{\square}$ is a solution for $\left\langle\Gamma ; W_{i} ; \sigma_{\square}\left(W_{i}\right)\right\rangle$ (for each $1 \leq$ $i \leq r)$ and for $\left\langle\Gamma ; R ; \sigma_{\square}\left(W^{\prime}\right)\right\rangle$ is evident. Next, suppose $p>0$. Put $\Delta^{\prime} \equiv \Delta, \forall w_{1}$ : $W_{1}, \ldots, \forall w_{r}: W_{r}$. Then we can write $v_{j}(1 \leq j \leq p)$ as $\operatorname{Deco}\left(v_{j}^{\prime} ; R_{j}\right)_{\Delta^{\prime}}$, for some $\lambda \tau$ term $v_{j}^{\prime}$. We have to show that $\sigma_{\square}$ is a solution for $\operatorname{SubMatch}\left(v_{j} ; R_{j}^{\prime}\right)_{\Delta^{\prime}, Z}$, where $R_{j}^{\prime}$ is either $R_{j}$ (if $u \in \operatorname{dom}(\Delta)$ ) or the twin of $R_{j}$ in $\sigma_{\square}\left(W_{i}\right)$ (if $u \equiv w_{i}$ ). Since $W_{i}$ is closed in $\Gamma$, we see that also in the second case $R_{j}^{\prime} \equiv R_{j}$. So in both cases $R_{j}^{\prime} \equiv R_{j} \equiv \sigma_{\square}\left(R_{j}\right)$. Thus it suffices to show hat $\sigma_{\square}$ is a solution for $\operatorname{SubMatch}\left(v_{j} ; \sigma_{\square}\left(R_{j}\right)\right)_{\Delta^{\prime}, Z}$. By assumption: $\sigma_{\square}(\Delta) \vdash_{\lambda \underline{\omega}} \lambda w_{1}: \sigma_{\square}\left(W_{1}\right) \ldots \lambda w_{r}: \sigma_{\square}\left(W_{r}\right) . u \sigma_{\square}\left(v_{1} \ldots v_{p}\right): \sigma_{\square}(W)$. From this it follows that $\sigma_{\square}\left(\Delta^{\prime}\right) \vdash_{\lambda \underline{\omega}} u \sigma_{\square}\left(v_{1}\right) \ldots \sigma_{\square}\left(v_{p}\right): \sigma_{\square}\left(W^{\prime}\right)$. In particular: $\sigma_{\square}\left(\Delta^{\prime}\right) \vdash_{\lambda \underline{\omega}} \sigma_{\square}\left(v_{j}\right): \sigma_{\square}\left(R_{j}\right)$, for every $1 \leq j \leq p$. To be able to apply the induction hypothesis we have yet to verify that $v_{j}$ is standard in $\Delta^{\prime}$ and $Z$, that $\left|v_{j}\right|$ is in LNF and that $R_{j}$ is of the form $R_{j}^{1} \rightarrow \cdots \rightarrow R_{j}^{p^{\prime}} \rightarrow R_{j}^{\prime}$, (for some $p^{\prime} \geq 0$ ) where every $R_{j}^{l}$ and $\sigma_{\square}\left(R_{j}^{\prime}\right)$ are closed in $\Gamma$. The first point follows from Lemma 5.27. As to the second point. If $u \in \operatorname{dom}(\Delta)$ then by standardness, $R_{j}$ is closed in $\Delta$ and hence in $\Gamma$. Next, suppose that $u \equiv x_{i}$. This case follows easily since $R_{j}$ is a subterm of $W_{i}$ and $W_{i}$ is closed in $\Gamma$. So we can apply the induction hypothesis: $\sigma_{\square}$ is a solution for $\operatorname{SubMatch}\left(v_{j} ; \sigma_{\square}\left(R_{j}\right)\right)_{\Delta^{\prime}, Z}$.

The second case is where $u \in Z$, say $u \equiv x_{i}$. Write $S_{i} \equiv R_{1} \rightarrow \cdots \rightarrow R_{m} \rightarrow R$ (note that $p=m)$. The new thing is that for each $1 \leq j \leq m$ and each $E$ in $\Phi_{x, i, j}$, we

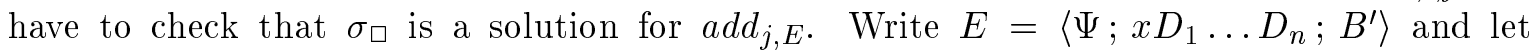
$E^{\prime}=\left\langle\Psi^{\prime} ; x D_{1}^{\prime} \ldots D_{n}^{\prime} ; B^{\prime \prime}\right\rangle$ be the triple in $\Phi\left(P, \sigma^{\star}\right)$ that corresponds to $E$. Then $\Psi^{\prime} \vdash_{\lambda \underline{\omega}}$ $D_{i}^{\prime}: \sigma_{\square}\left(R_{1}\right) \rightarrow \cdots \rightarrow \sigma_{\square}\left(R_{m}\right) \rightarrow \sigma_{\square}(R)$.

Again, we distinguish two cases. First suppose that $D_{i} \equiv \lambda y_{1}: O \ldots \lambda y_{m}: O . y_{j}$. Since $\left|D_{i}^{\prime}\right| \equiv D_{i}$, we have that $D_{i}^{\prime} \equiv \lambda y_{1}: \sigma_{\square}\left(R_{1}\right) \ldots \lambda y_{m}: \sigma_{\square}\left(R_{m}\right) . y_{j}$. So obviously $\sigma_{\square}$ is a solution for $\left\langle\Gamma ; R_{j} ; \sigma_{\square}(R)\right\rangle$ and since by assumption $\sigma_{\square}(R) \equiv \sigma_{\square}\left(W^{\prime}\right), \sigma_{\square}$ is a solution for $\left\langle\Gamma ; R_{j} ; \sigma_{\square}\left(W^{\prime}\right)\right\rangle$.

We have to show that $\sigma_{\square}$ is a solution for $\operatorname{SubMatch}\left(v_{j} ; \sigma_{\square}\left(W^{\prime}\right)\right)_{\Delta^{\prime}, Z}$. First observe that $\sigma_{\square}\left(W^{\prime}\right) \equiv \sigma_{\square}\left(R_{j}\right)$. So this case is similar to the one treated above. The verification of the fact that the induction hypothesis is applicable is actually easier than before because $R_{j}$ is atomic.

Next suppose that $D_{i} \equiv \lambda y_{1}: O \ldots \lambda y_{m}: O \cdot e s_{1} \ldots s_{l}\left(e \not \equiv y_{j}\right.$, for $\left.1 \leq j \leq m\right)$. We can identify some types. It must hold that $\Psi^{\prime} \vdash_{\lambda \underline{\omega}} e: Q_{1} \rightarrow \cdots \rightarrow Q_{l} \rightarrow Q$, for some $Q_{1}, \ldots, Q_{l}$, $Q$ such that $\sigma_{\square}(R) \equiv Q$. Recall that by assumption $\sigma_{\square}\left(W^{\prime}\right) \equiv \sigma_{\square}(R)$. Let $\Psi_{1}$ be the $\lambda \underline{\omega}$-companion to $\Psi$ and $\Psi_{2}$ be $\Psi_{1}, \forall y_{1}: R_{1}, \ldots, \forall y_{m}: R_{m}$. By Lemma 5.22 , we have that $\sigma_{\square}\left(\Psi_{1}\right)$ is an initial segment of $\Psi^{\prime}$. We have to show that $\sigma_{\square}$ is a solution for $\left\langle\Gamma ; R_{j} ; U\right\rangle$ where $U \in \operatorname{Targets}\left(y_{j} ; \operatorname{Deco}\left(e s_{1} \ldots s_{l} ; \sigma_{\square}\left(W^{\prime}\right)\right)_{\Psi_{2}} ; \sigma_{\square}\left(W^{\prime}\right)\right)_{\Psi_{2}}$. 
Since $\sigma_{\square}\left(W^{\prime}\right) \equiv \sigma_{\square}(R)$ it suffices to show that $\sigma_{\square}$ is a solution for $\left\langle\Gamma ; R_{j} ; U\right\rangle$ where $U \in \operatorname{Targets}\left(y_{j} ; \operatorname{Deco}\left(e s_{1} \ldots s_{l} ; \sigma_{\square}(R)\right)_{\Psi_{2}} ; \sigma_{\square}(R)\right)_{\Psi_{2}}$.

Let $\Psi^{\prime \prime}$ be $\Psi^{\prime}, \forall y_{1}: \sigma_{\square}\left(R_{1}\right), \ldots, \forall y_{m}: \sigma_{\square}\left(R_{m}\right)$. Since $\left|D_{i}^{\prime}\right| \equiv D_{i}$, we have that $D_{i}^{\prime} \equiv$ $\lambda y_{1}: \sigma_{\square}\left(R_{1}\right) \ldots \lambda y_{m}: \sigma_{\square}\left(R_{m}\right) . g$ for some term $g$ such that $|g| \equiv e s_{1} \ldots s_{l}$. So we have $\Psi^{\prime \prime} \vdash_{\lambda \underline{\omega}}$ $g: \sigma_{\square}(R)$ and by Lemma 5.35, $\Psi^{\prime \prime} \vdash_{\lambda \underline{\omega}} \operatorname{Deco}\left(e s_{1} \ldots s_{l} ; \sigma_{\square}(R)\right)_{\Psi^{\prime \prime}}: \sigma_{\square}(R)$.

So by Lemma 5.40, Targets $\left(y_{j} ; \operatorname{Deco}\left(e s_{1} \ldots s_{l} ; \sigma_{\square}(R)\right)_{\Psi^{\prime \prime}} ; \sigma_{\square}(R)\right)_{\Psi^{\prime \prime}}=\left\{\Psi^{\prime \prime}\left(y_{j}\right)\right\}=\sigma_{\square}\left(R_{j}\right)$. Hence we are done if we can show that Targets $\left(y_{j} ; \operatorname{Deco}\left(e s_{1} \ldots s_{l} ; \sigma_{\square}(R)\right)_{\Psi_{2}} ; \sigma_{\square}(R)\right)_{\Psi_{2}}=$ $\operatorname{Targets}\left(y_{j} ; \operatorname{Deco}\left(e s_{1} \ldots s_{l} ; \sigma_{\square}(R)\right)_{\Psi^{\prime \prime}} ; \sigma_{\square}(R)\right)_{\Psi^{\prime \prime}}$.

By Lemma 5.41 (2), it suffices to show that for all $z, z$ free in $e s_{1} \ldots s_{l}$ such that $\Psi_{2}(z)$ is not atomic we have: $\Psi_{2}(z) \equiv \Psi^{\prime \prime}(z)$ (it is easy to check that the other conditions mentioned in that lemma are satisfied). Consider such a $z$. Since its type is not atomic, $z$ is not among $\left\{y_{1}, \ldots, y_{m}\right\}$. Thus, by a similar application of Lemma 2.11 as in the proof of Lemma 5.14, we have that $z$ occurs in $B^{\prime}$, hence (by Lemma 5.24) is of closed type in $\Psi_{1}$. So $\Psi_{2}(z) \equiv$ $\Psi_{1}(z) \equiv \sigma_{\square}\left(\Psi_{1}(z)\right) \equiv \Psi^{\prime}(z) \equiv \Psi^{\prime \prime}(z)$. That $\sigma_{\square}$ is a solution for the remaining part of $a d d_{j, E}$ is verified as before.

The completeness of the algorithm presented in the proof of Theorem 5.54 is obtained by showing that if $P$ in $\lambda \underline{\omega}$ has solution $\sigma$ then $\sigma_{\square}$ is a solution for Match for all heads $x$ in $\Phi\left(|P|,|\sigma|^{\star}\right)$. The proof of this fact proceeds by induction on the depth of heads in $\Phi\left(|P|,|\sigma|^{\star}\right)$. The following lemma is needed for the induction step.

Lemma 5.53. Let $P=\langle\Gamma ; A ; B\rangle$ be a third-order matching problem for objects in $\lambda \underline{\omega}$ and $\sigma$ a solution for $P$. Let $x$ be a head in $\Phi\left(|P|,|\sigma|^{\star}\right)$ of depth $n$ and of type $T_{1} \rightarrow \cdots \rightarrow T_{n} \rightarrow T$ in $\Gamma$. Let $y$ be a head below $x$ of depth $n+1$ and of type $S_{1} \rightarrow \cdots \rightarrow S_{m} \rightarrow S$ in $\Gamma$, where $S$ is not closed in $\Gamma$. Suppose that $\theta$ is a $\square$-substitution, well-typed in $\Gamma$, such that $\theta(T) \equiv \sigma_{\square}(T)$ and $\theta(T)$ is closed in $\Gamma$. Then Match $(x, \theta, n)_{\Gamma}$ contains a matching problem $\left\langle\Gamma ; S ; \sigma_{\square}(S)\right\rangle$.

Proof. Let $E=\left\langle\Psi ; x D_{1} \ldots D_{n} ; C\right\rangle \in \Phi\left(|P|,|\sigma|^{\star}\right)$. We can write $\Psi$ as $\Psi_{1}, \Psi_{2}$, where $\Psi_{2}$ consists of dummy variables added in the construction of $\Phi\left(|P|,|\sigma|^{\star}\right)$. Let $x A_{1} \ldots A_{n}$ be the witnessing term of $E$ in $\Phi\left(|P|,|\sigma|^{\star}\right)$. By Lemma 5.22, $\Psi_{1} \vdash_{\lambda \tau} x A_{1} \ldots A_{n}: O$ and $|\sigma|^{\star}\left(x A_{1} \ldots A_{n}\right)={ }_{\beta \eta} C$. Moreover each $D_{i}(1 \leq i \leq n)$ is either a dummy variable or $|\sigma|^{\star}\left(A_{i}\right)$. In the second case we know that $z_{i}$ occurs in the normal form of $|\sigma|^{\star}(x)|\sigma|^{\star}\left(A_{1}\right) \ldots|\sigma|^{\star}\left(A_{i-1}\right) z_{i}|\sigma|^{\star}\left(A_{i+1}\right) \ldots|\sigma|^{\star}\left(A_{n}\right) \quad\left(z_{i}\right.$ a fresh variable of the appropriate type). By assumption, we know that for some $1 \leq i \leq n$, the second case applies, $D_{i} \equiv$ $|\sigma|^{\star}\left(A_{i}\right)$ and $y \in F V\left(A_{i}\right)$. So $y$ is a head in $\Phi\left(\left\langle\Psi^{\prime} ; z t_{1} \ldots t_{p} ; D_{i}^{\prime}\right\rangle,|\sigma|^{\star}\right)$, where we write $A_{i} \equiv$ $\lambda u_{1}: U_{1} \ldots \lambda u_{l}: U_{l} . z t_{1} \ldots t_{p}, D_{i} \equiv \lambda u_{1}: U_{1} \ldots \lambda u_{l}: U_{l} . D_{i}^{\prime}$ and $\Psi^{\prime} \equiv \Psi_{1}, \forall u_{1}: U_{1}, \ldots, \forall u_{l}: U_{l}$. We distinguish four cases, three of which turn out to be impossible.

1. $z \equiv u_{j}$, for some $1 \leq j \leq l$. Because of typing reasons $p=0$, contradicting the assumption that $y \in F V\left(A_{i}\right)$.

2. $z$ is existential in $\Psi, z \not \equiv y$. Then $y$ is not of depth $n+1$ and the result follows. 
3. $z$ is universal in $\Psi$. We show that $y$ cannot be of depth $n+1$, contradicting the assumption. To $E$ corresponds by Proposition 5.29, a triple $F=\left\langle\Psi^{\prime \prime} ; x D_{1}^{\prime \prime} \ldots D_{n}^{\prime \prime} ; C^{\prime \prime}\right\rangle$ in $\Phi\left(P, \sigma^{\star}\right)$. Let $x A_{1}^{\prime \prime} \ldots A_{n}^{\prime \prime}$ be the witnessing term of $F$ in $\Phi\left(P, \sigma^{\star}\right)$. By Lemma 5.23 we know that $\left|x A_{1}^{\prime \prime} \ldots A_{n}^{\prime \prime}\right| \equiv x A_{1} \ldots A_{n}$. By Lemma 5.22 , we have that $\sigma^{\star}\left(x A_{1}^{\prime \prime} \ldots A_{n}^{\prime \prime}\right)={ }_{\beta \eta}$ $C^{\prime \prime}$ and we have that $z_{i}^{\prime \prime}\left(z_{i}^{\prime \prime}\right.$ a fresh variable of the appropriate type) occurs in the normal form of $\sigma^{\star}(x) \sigma^{\star}\left(A_{1}^{\prime \prime}\right) \ldots \sigma^{\star}\left(A_{i-1}^{\prime \prime}\right) z_{i}^{\prime \prime} \sigma^{\star}\left(A_{i+1}^{\prime \prime}\right) \ldots \sigma^{\star}\left(A_{n}^{\prime \prime}\right)$.

Write $A_{i}^{\prime \prime} \equiv \lambda u_{1}: U_{1}^{\prime \prime} \ldots \lambda u_{l}: U_{l}^{\prime \prime} . z t_{1}^{\prime \prime} \ldots t_{p}^{\prime \prime}$. Let $\Xi$ be the $\lambda \underline{\omega}$-companion to $\Psi$ and let $\Xi^{\prime}$ be $\Xi, \forall u_{1}: U_{1}^{\prime \prime}, \ldots, \forall u_{l}: U_{l}^{\prime \prime}$. Using Lemma 5.22 , we see that $\Xi^{\prime} \vdash_{\lambda \underline{\omega}} z t_{1}^{\prime \prime} \ldots t_{p}^{\prime \prime}: Z$ for some term $Z$. By Lemma 2.11, $\sigma^{\star}\left(z t_{1}^{\prime \prime} \ldots t_{p}^{\prime \prime}\right)$ is closed in $\sigma^{\star}\left(\Xi^{\prime}\right)$. (A pply this lemma with $\sigma^{\star}(\Xi)$ for $\Gamma,\langle\rangle$ for $\Delta, z_{i}^{\prime \prime}$ for $x, \sigma^{\star}(x) \sigma^{\star}\left(A_{1}^{\prime \prime}\right) \ldots \sigma^{\star}\left(A_{i-1}^{\prime \prime}\right) z_{i}^{\prime \prime} \sigma^{\star}\left(A_{i+1}^{\prime \prime}\right) \ldots \sigma^{\star}\left(A_{n}^{\prime \prime}\right)$ for $A, \sigma^{\star}\left(A_{i}^{\prime \prime}\right)$ for $B$ and the normal form of $C^{\prime \prime}$ for $\left.C\right)$. Moreover every variable, free in $\sigma^{\star}\left(z t_{1}^{\prime \prime} \ldots t_{p}^{\prime \prime}\right)$ and of non-atomic type in $\Xi^{\prime}$ (or in $\sigma^{\star}\left(\Xi^{\prime}\right)$ ), which is therefore not among $\left\{u_{1}, \ldots, u_{l}\right\}$, occurs in $C^{\prime \prime}$. By Lemma 5.24, such a variable has closed type in $\Xi$ and thus in $\Xi^{\prime}$. By Lemma 3.11, taking $z t_{1}^{\prime \prime} \ldots t_{p}^{\prime \prime}$ for $A, \Xi^{\prime}$ for $\Gamma$ and $y$ for $z$, every occurrence of $y$ must be in the scope of another existential variable bound by $\sigma^{\star}$ and hence by $|\sigma|^{\star}$. We may conclude that $y$ cannot be of depth $n+1$.

4. $z \equiv y$. Let $|\sigma|^{\star}(x)$ be $\lambda v_{1}: V_{1} \ldots \lambda v_{n}: V_{n} . w s_{1} \ldots s_{r}$. By construction of $\Phi\left(P,|\sigma|^{\star}\right)$, $v_{i} \in F V\left(w s_{1} \ldots s_{r}\right)$ (otherwise $y$ would not be a head in $\Phi\left(\left\langle\Psi^{\prime} ; z t_{1} \ldots t_{p} ; D_{i}^{\prime}\right\rangle\right)$ ). Let $\operatorname{Deco}\left(|\sigma|^{\star}(x) ; T_{1} \rightarrow \cdots \rightarrow T_{n} \rightarrow \theta(T)\right)_{\Gamma}$ be $\lambda v_{1}: T_{1} \ldots \lambda v_{n}: T_{n} . w s_{1}^{\prime} \ldots s_{r}^{\prime}$. Again we have $v_{i} \in$ $F V\left(w s_{1}^{\prime} \ldots s_{r}^{\prime}\right)$. Write $T_{i} \equiv R_{1} \rightarrow \cdots \rightarrow R_{l} \rightarrow R$. One easily verifies that $S \equiv R$ (and hence $\left.\sigma_{\square}(R) \equiv \sigma_{\square}(S)\right)$. By Lemma 5.48 we have that $\operatorname{Match}(x, \theta, n)_{\Gamma}$ contains a matching problem $\left\langle\Gamma ; R ; R^{\prime}\right\rangle$ for some term $R^{\prime}$. Now recall that $\theta(T) \equiv \sigma_{\square}(T)$, closed in $\Gamma$. So $\operatorname{Match}(x, \theta, n)_{\Gamma}=\operatorname{Match}\left(x, \sigma_{\square}, n\right)_{\Gamma}$. By Lemma 5.52, $\sigma_{\square}$ is a solution for $\operatorname{Match}\left(x, \sigma_{\square}, n\right)_{\Gamma}$. So $R^{\prime} \equiv \sigma_{\square}(R)$. We are done.

At last we have reached the point where we can state and prove our main result.

Theorem 5.54. It is decidable whether a third-order matching problem for objects in $\lambda \underline{\omega}$ has a solution or not.

Proof. Let $P=\langle\Gamma ; A ; B\rangle$ be a third-order matching problem for objects in $\lambda \underline{\omega}$. We present an algorithm which, given $P$ as input, returns a solution for $P$ if $P$ has a solution and fail otherwise. The algorithm is as follows.

- Translate $P$ to $|P|$.

- Enumerate the solutions in $\operatorname{Sol}(|P|)$ as $\left\{\sigma_{i} \mid 1 \leq i \leq n\right\}$. If $\operatorname{Sol}(|P|)$ is empty, return fail and stop. Else do the following.

- Let $\alpha$ be a meta variable ranging over substitutions and dummy some dummy substitution. Put $i:=1$ and $\alpha:=d u m m y$. While $i \leq n$ do 
- Delete all triples $\langle x ; \gamma ; t\rangle$ in $\sigma_{i}$ such that $x$ is not a head in $\Phi\left(|P|, \sigma_{i}\right)$. (By Lemma 5.17, the resulting substitution, which we keep denoting by $\sigma_{i}$, is still a solution for $|P|$ and $\left.\Phi\left(|P|, \sigma_{i}\right)\right)$. If the result is the substitution $\emptyset$, put $i:=n+1$ and $\alpha:=\emptyset$. Else do the following.

- Define $\operatorname{appr}(0):=\{\emptyset\} ; \operatorname{appr}(k+1):=\bigcup_{\theta \in \operatorname{appr}(k)} \operatorname{Sol}\left(\operatorname{Match}\left(\sigma_{i}, \theta, k\right)_{\Gamma}\right)$

- Let $d$ be the depth of $\Phi\left(|P|, \sigma_{i}\right)$. If there exist a $\theta_{1}$ in $\operatorname{appr}(d)$ such that for all $x$, head in $\Phi\left(|P|, \sigma_{i}\right)$ (with $\Gamma(x) \equiv S_{1} \rightarrow \cdots \rightarrow S_{n} \rightarrow S$ ), $\theta_{1}(S)$ is closed in $\Gamma$ and if there exists a $\theta_{2}$ in $\operatorname{Sol}\left(\operatorname{Match}\left(\sigma_{i}, \theta_{1}, d\right)_{\Gamma}\right)$, put $i:=n+1$ and $\alpha:=\theta_{2} \circ \operatorname{Deco}\left(\sigma_{i}\right)_{\Gamma}$, else put $i:=i+1$.

- If $\alpha \equiv d u m m y$, return fail, else return $\alpha$. Stop.

We have to check that this algorithm is sound and complete and that it always terminates.

Soundness. Let $\sigma$ be a standard solution for $|P|$ in $S o l(|P|)$ and $d$ the depth of $\Phi(|P|, \sigma)$. If, after deletion of superfluous parts, $\sigma=\emptyset$, then we must have that $|A| \equiv|B|$, and $\emptyset$ is by Lemma 5.12 (2) indeed a solution for $P$. Next, suppose that $\sigma \neq \emptyset$. Then by Lemma 3.7, there must be an $x \in F V(A)$, bound by $\sigma$ and of type $S_{1} \rightarrow \cdots \rightarrow S_{n} \rightarrow S$ in $\Gamma$, with $S$ is closed in $\Gamma$. Let $\tau_{1}$ be an element of $\operatorname{appr}(d)$ such that $\tau_{1}(S)$ is closed in $\Gamma$ for all $x$, head in $\Phi(|P|, \sigma)$ (with $\left.\Gamma(x) \equiv S_{1} \rightarrow \cdots \rightarrow S_{n} \rightarrow S\right)$. Let $\tau_{2}$ be an element of $\operatorname{Sol}\left(\operatorname{Match}\left(\sigma, \tau_{1}, d\right)_{\Gamma}\right)$. We show that $\tau_{2} \circ \operatorname{Deco}(\sigma)_{\Gamma}$ is a solution for $P$. By construction there exist substitutions $\rho_{1}, \ldots$, $\rho_{d-1}$ such that $\rho_{1}$ is a standard solution for $\operatorname{Match}(\sigma, \emptyset, 0)_{\Gamma}$, each $\rho_{i+1}$ is a standard solution for $\operatorname{Match}\left(\sigma, \rho_{i}, i\right)_{\Gamma}$ and $\tau_{1}$ is a standard solution for $\operatorname{Match}\left(\sigma, \rho_{d-1}, d-1\right)_{\Gamma}$. By Lemma 5.49 each $\rho_{i}$ is well-typed in $\Gamma$. The same holds for $\tau_{1}$ and $\tau_{2}$. By Lemma 5.49 and Corollary 5.51, $\tau_{2} \circ \operatorname{Deco}(\sigma)_{\Gamma}$ is well-typed in $\Gamma$. By Lemma 5.34, $\left|\tau_{2} \circ \operatorname{Deco}(\sigma)_{\Gamma}\right|=\sigma$ and hence $\left|\tau_{2} \circ D e c o(\sigma)_{\Gamma}\right|$ is a solution for $|P|$. By Lemma 5.13, $\tau_{2} \circ \operatorname{Deco}(\sigma)_{\Gamma}$ is a solution for $P$.

Completeness. Suppose $P$ has a solution $\sigma$. We have to prove that the algorithm returns a substitution. It is no restriction to assume that $\operatorname{dom}(\sigma) \subseteq \operatorname{dom}(\Gamma)$. By Proposition 5.11, $|\sigma|$ is a solution for $|P|$. So $|\sigma|^{\star}$ is also a solution for $|P|$ and occurs in the first enumeration given by the algorithm. The interesting case is where the deleting of superfluous parts from $|\sigma|^{\star}$ does not yield the empty substitution. Let $d>0$ be the depth of $\Phi\left(|P|,|\sigma|^{\star}\right)$. Completeness follows from the next claim, which implies that $\alpha:=\theta_{d} \circ \operatorname{Deco}\left(|\sigma|^{\star}\right)_{\Gamma}$ is a solution for $P$.

Claim. For each $k, 0 \leq k \leq d$, there exist a $\zeta_{k} \in \operatorname{appr}(k)$ and a $\theta_{k} \in \operatorname{appr}(k+1)$ such that $\theta_{k} \in \operatorname{Sol}\left(\operatorname{Match}\left(|\sigma|^{\star}, \zeta_{k}, k\right)_{\Gamma}\right)$ and for all heads $x$ of depth $l \leq k$ in $\Phi\left(|P|,|\sigma|^{\star}\right)$ and of type $S_{1} \rightarrow \cdots \rightarrow S_{n} \rightarrow S$ in $\Gamma, \zeta_{k}(S) \equiv \theta_{k}(S) \equiv \sigma_{\square}(S)$ (and these types are closed in $\Gamma$ ).

Proof of claim. By induction on $k$.

- $k=0$. Note that if $x$ is a variable of depth 0 and of type $S_{1} \rightarrow \cdots \rightarrow S_{n} \rightarrow S$ in $\Gamma$ then $S$ is closed in $\Gamma$. Take $\zeta_{0}=\emptyset$. By Lemma 5.52, $\sigma_{\square}$ is a solution for $\operatorname{Match}(x, \emptyset, 0)_{\Gamma}$ and hence $\left(\sigma_{\square}\right)^{\star} \in \operatorname{appr}(1)$ is also a solution for $\operatorname{Match}(x, \emptyset, 0)_{\Gamma}$. Since $S$ is closed in $\Gamma$, $\emptyset(S) \equiv\left(\sigma_{\square}\right)^{\star}(S) \equiv \sigma_{\square}(S) \equiv S$.

- $k=k^{\prime}+1$. We put $\zeta_{k^{\prime}+1}:=\theta_{k^{\prime}}$.

First we show that $\operatorname{Match}\left(|\sigma|^{\star}, \theta_{k^{\prime}}, k^{\prime}+1\right)_{\Gamma} \subseteq \operatorname{Match}\left(|\sigma|^{\star}, \sigma_{\square}, k^{\prime}+1\right)_{\Gamma}$. The interesting case is where there exists a head $y$ of depth at most $k^{\prime}+1$ in $\Phi\left(|P|,|\sigma|^{\star}\right)$ and of type 
$T_{1} \rightarrow \cdots \rightarrow T_{m} \rightarrow T$ in $\Gamma$, where $T$ is not closed in $\Gamma$. There exists a head $z$ above $y$ in $\Phi\left(|P|,|\sigma|^{\star}\right)$ whose depth is $k^{\prime}$. Let $Z_{1} \rightarrow \cdots \rightarrow Z_{l} \rightarrow Z$ be the type of $z$ in $\Gamma$. The induction hypothesis yields that $\zeta_{k^{\prime}}(Z) \equiv \sigma_{\square}(Z)$ is closed in $\Gamma$. Hence $\theta_{k^{\prime}}$ is a solution for Match $\left(z, \zeta_{k^{\prime}}, k^{\prime}\right)_{\Gamma}$. By Lemma 5.53 there exists a matching problem $\left\langle\Gamma ; T ; \sigma_{\square}(T)\right\rangle \in$ $\operatorname{Match}\left(z, \zeta_{k^{\prime}}, k^{\prime}\right)_{\Gamma}$. So $\theta_{k^{\prime}}(T) \equiv \sigma_{\square}(T)$, by construction closed in $\Gamma$.

Thus Match $\left(|\sigma|^{\star}, \theta_{k^{\prime}}, k^{\prime}+1\right)_{\Gamma} \subseteq \operatorname{Match}\left(|\sigma|^{\star}, \sigma_{\square}, k^{\prime}+1\right)_{\Gamma}$. So by Lemma 5.52, $\sigma_{\square}$ is a solution for $\operatorname{Match}\left(|\sigma|^{\star}, \theta_{k^{\prime}}, k^{\prime}+1\right)_{\Gamma}$, hence there is a $\theta_{k^{\prime}+1}$ in $\operatorname{Sol}\left(\operatorname{Match}\left(|\sigma|^{\star}, \theta_{k^{\prime}}, k^{\prime}+1\right)_{\Gamma}\right)$.

We still have to check that for all heads $x$ of depth $l \leq k^{\prime}+1$ in $\Phi\left(|P|,|\sigma|^{\star}\right)$ and of type $S_{1} \rightarrow \cdots \rightarrow S_{n} \rightarrow S$ in $\Gamma, \theta_{k^{\prime}}(S) \equiv \theta_{k^{\prime}+1}(S) \equiv \sigma_{\square}(S)$, closed in $\Gamma$. For all heads of depth $l \leq k^{\prime}$ we know by induction hypothesis that $\theta_{k^{\prime}}(S) \equiv \sigma_{\square}(S)$, closed in $\Gamma$. Furthermore, we have already proved that if $x$ is a head of depth $k^{\prime}+1$, then $\theta_{k^{\prime}}(S) \equiv \sigma_{\square}(S)$, closed in $\Gamma$. So for all heads $x$ of depth $l \leq k^{\prime}+1$ we have $\theta_{k^{\prime}}(S) \equiv \sigma_{\square}(S)$, closed in $\Gamma$. Since $\theta_{k^{\prime}+1} \in \operatorname{Sol}\left(\operatorname{Match}\left(|\sigma|^{\star}, \theta_{k^{\prime}}, k^{\prime}+1\right)_{\Gamma}\right), \theta_{k^{\prime}+1}$ is a solution for $\left\langle\Gamma ; S ; \theta_{k^{\prime}}(S)\right\rangle$, hence $\theta_{k^{\prime}+1}(S) \equiv \theta_{k^{\prime}}(S)$, closed in $\Gamma$.

Termination. Evidently, $|P|$ can be computed in time linear in the length of $P$. By Theorem 5.28, $\operatorname{Sol}(|P|)$ can be enumerated in time bounded by some function depending only on $|P|$. The decoration procedure for each solution $\sigma_{i}$ can be performed in time linear in the number of symbols in $P$ and $\sigma_{i}$. For all standard solutions $\sigma_{i}$ for $|P|$ and all substitutions $\rho$, well-typed in $\Gamma$, the number of matching problems and the length of each matching problem in $\operatorname{Match}\left(\sigma_{i}, \rho, d\right)_{\Gamma}$ is linear in the number of symbols in $\sigma_{i}$. Since moreover $\operatorname{Match}\left(\sigma_{i}, \rho, d\right)_{\Gamma}$ is a collection of third-order matching problem for types in $\lambda \underline{\omega}, \operatorname{Sol}\left(\operatorname{Match}\left(\sigma_{i}, \rho, d\right)_{\Gamma}\right)$ can again be enumerated in time bounded by some function depending only on $\operatorname{Match}\left(\sigma_{i}, d, \rho\right)_{\Gamma}$. So at each stage there are finitely many new matching problems. Because the depth of $\Phi(P, \sigma)$ is finite there are finitely many such stages.

\section{Third-order closed matching is undecidable in $\lambda \underline{\omega}$}

In this section we show that it is undecidable whether a third-order matching problem in $\lambda \underline{\omega}$ has a closed solution or not. The proof is a slightly more complicated variant of the proofs in [5]. It is noteworthy that the proof does not depend on the undecidability of the problem whether there is a term of a given type (in a given context). Indeed, this problem is decidable (a proof of this fact will appear in the author's forthcoming thesis).

Definition 6.1. A unification problem $\langle\Gamma ; A ; B\rangle$ in $\lambda \underline{\omega}$ is called elementary at the level of types (or elementary, for short) if:

- $\Gamma \equiv\langle\forall Z: *\rangle, \Gamma^{\prime}$, for some variable $Z$.

- for all declarations $Q x: T$ in $\Gamma$ it holds that $T \in\{*, * \rightarrow *, * \rightarrow * \rightarrow *, * \rightarrow * \rightarrow * \rightarrow *\}$. (Note that the order of these kinds is at most three.) 
- $\Gamma \vdash_{\lambda \underline{\omega}} A: *$ and $\Gamma \vdash_{\lambda \underline{\omega}} B: *$.

Theorem 6.2 (Goldfarb, Dowek). There exists no algorithm that decides whether a elementary unification problem $\langle\Gamma ; A ; B\rangle$ in $\lambda \underline{\omega}$ has a (closed) solution or not.

Proof. See [4], [14]. The assumption that $\Gamma$ starts with the declaration $\forall Z: *$ is justified by the fact that Goldfarb assumes that the language for which the unification problem is stated contains two individual constants (although he does not need this assumption).

Remark 6.3. It is no restriction to assume that if an elementary unification problem $\langle\Gamma ; A ; B\rangle$ in $\lambda \underline{\omega}$ has a solution then $\sigma(A)$ (and hence $\sigma(B)$ ) is closed in $\sigma(\Gamma)$. For, let $\sigma$ be a solution and let $e$ be an existential variable occurring in $\sigma(A)$. In case $e$ has type $*$, we can extend $\sigma$ with $\{\langle e ;\langle\rangle ; Z\rangle\}$; in case $e$ has type $* \rightarrow *$, we can extend $\sigma$ with $\{\langle e ;\langle\rangle ; \lambda x: * . Z\rangle\}$, etc.

Theorem 6.4. There exists no algorithm that decides whether a third-order matching problem in $\lambda \underline{\omega}$ has a closed solution or not.

Proof. For every elementary unification problem $\left\langle\Gamma_{1} ; A_{1} ; B_{1}\right\rangle$ in $\lambda \underline{\omega}$ we construct a matching problem $\left\langle\Gamma_{2} ; A_{2} ; B_{2}\right\rangle$ for objects in $\lambda \underline{\omega}$ such that the order of the existential variables in $\Gamma_{2}$ is at most 3 and such that $\left\langle\Gamma_{1} ; A_{1} ; B_{1}\right\rangle$ has a closed solution iff $\left\langle\Gamma_{2} ; A_{2} ; B_{2}\right\rangle$ has a closed solution. The result then follows from Theorem 6.2.

Let $\left\langle\Gamma_{1} ; A_{1} ; B_{1}\right\rangle$ be given. We define:

- $\Gamma_{2} \equiv \Gamma_{1}, \forall X: * \rightarrow *, \forall c: Z, \forall d: Z, \forall g: Z \rightarrow Z \rightarrow Z, \forall b: X B_{1}$, $\exists f:\left(\left(X A_{1}\right) \rightarrow Z\right) \rightarrow\left(X B_{1}\right) \rightarrow Z$.

(Here the new variables are fresh.)

- $A_{2} \equiv g\left(f\left(\lambda z: X A_{1} . c\right) b\right)\left(f\left(\lambda z: X A_{1} . d\right) b\right)$.

- $B_{2} \equiv g c d$.

This defines a third-order matching problem for objects in $\lambda \underline{\omega}$. We show that closed solutions for $\left\langle\Gamma_{1} ; A_{1} ; B_{1}\right\rangle$ are in bijective correspondence with closed solutions for $\left\langle\Gamma_{2} ; A_{2} ; B_{2}\right\rangle$.

Suppose $\sigma$ is a solution for $\left\langle\Gamma_{1} ; A_{1} ; B_{1}\right\rangle$, i.e. $\sigma\left(A_{1}\right)={ }_{\beta \eta} \sigma\left(B_{1}\right)$. From $\sigma$ we construct a solution $\tau$ for $\left\langle\Gamma_{2} ; A_{2} ; B_{2}\right\rangle$ :

$$
\tau=\sigma \cup\left\{\left\langle f ;\langle\rangle ; \lambda x:\left(X \sigma\left(A_{1}\right)\right) \rightarrow Z . \lambda y: X \sigma\left(B_{1}\right) . x y\right\rangle\right\} .
$$

We have to verify three things.

1. $\tau\left(A_{2}\right)={ }_{\beta \eta} B_{2}$. This is easy.

2. $\tau$ is closed. We know that $\sigma$ is closed. By Remark 6.3, we may assume that $\sigma\left(A_{1}\right)$ and $\sigma\left(B_{1}\right)$ are closed in $\sigma\left(\Gamma_{1}\right)$ and hence in $\tau\left(\Gamma^{\prime}\right)$. So $\lambda x:\left(X \sigma\left(A_{1}\right)\right) \rightarrow Z . \lambda y: X \sigma\left(B_{1}\right) . x y$ is closed in $\tau\left(\Gamma^{\prime}\right)$ from which we may conclude that $\tau$ is closed.

3. $\tau$ is well-typed in $\Gamma_{2}$. This consists of three things: 
(a) We must show that $\tau$ binds no variable that are universal in $\Gamma_{2}$. This is easy, since $\sigma$ has this property w.r.t. $\Gamma_{1}$.

(b) We must show that $\tau\left(\Gamma_{2}\right)$ is legal. This is proved by induction on the length of $\Gamma_{2}$, using that $\sigma\left(\Gamma_{1}\right)$ is legal and that $\sigma\left(\Gamma_{1}\right) \vdash_{\lambda \underline{\omega}} \sigma\left(B_{1}\right): *$.

(c) For all existential variables $x$ in $\Gamma_{2}$ bound by $\tau$ (say $\langle x ; \gamma ; M\rangle \in \tau$ ), we must show that $\tau\left(\left(\Gamma_{2}\right)_{x}\right), \gamma \vdash_{\lambda \circ} M: \tau\left(\left(\Gamma_{2}\right)_{x}\right)$. We treat the only new case, the existential variable $f$. We have to show that

$\tau\left(\left(\Gamma_{2}\right)_{f}\right) \vdash_{\lambda \underline{\omega}} \lambda x:\left(X \sigma\left(A_{1}\right)\right) \rightarrow Z . \lambda y: X \sigma\left(B_{1}\right) . x y:\left(\left(X \tau\left(A_{1}\right)\right) \rightarrow Z\right) \rightarrow\left(X \tau\left(B_{1}\right)\right) \rightarrow Z$.

But this is trivial, given the fact that $\tau\left(A_{1}\right)={ }_{\beta \eta} \sigma\left(A_{1}\right)={ }_{\beta \eta} \sigma\left(B_{1}\right)={ }_{\beta \eta} \tau\left(B_{1}\right)$.

Conversely, suppose that $\left\langle\Gamma_{2} ; A_{2} ; B_{2}\right\rangle$ has a closed solution $\tau$. Then $\tau$ is well-typed in $\Gamma_{2}$ and (since $\Gamma_{1}$ is an initial segment of $\Gamma_{2}$ ) $\tau$ is also well-typed in $\Gamma_{1}$. Next we show (using $\left.\tau\left(A_{2}\right)={ }_{\beta \eta} B_{2}\right)$ that $\tau\left(A_{1}\right)={ }_{\beta \eta} \tau\left(B_{1}\right)$.

Put $\Delta \equiv\left(\tau\left(\Gamma_{2}\right)\right), \forall y_{1}:\left(X \tau\left(A_{1}\right)\right) \rightarrow Z, \forall y_{2}: X \tau\left(B_{1}\right)$ and define $v$ as the $\beta \eta$-normal form of $(\tau(f)) y_{1} y_{2}$. We have that

$$
\Delta \vdash^{\lambda \underline{\omega}}(\tau(f)) y_{1} y_{2}: Z
$$

and so by Subject Reduction:

$$
\Delta \vdash_{\lambda \underline{\omega}} v: Z \text {. }
$$

Below we show that $v \equiv y_{1} y_{2}$ or $v \equiv y_{1} b$. For typing reasons this is only possible when $X \tau\left(A_{1}\right)={ }_{\beta \eta} X \tau\left(B_{1}\right)$, which is equivalent to $\tau\left(A_{1}\right)={ }_{\beta \eta} \tau\left(B_{1}\right)$.

The equation $\tau\left(A_{2}\right)={ }_{\beta \eta} B_{2}$ is by Substitutivity equivalent to the system consisting of the following two equations:

$$
\begin{aligned}
& v\left[y_{1}:=\lambda z: X \tau\left(A_{1}\right) \cdot c, y_{2}:=b\right]={ }_{\beta \eta} c . \\
& v\left[y_{1}:=\lambda z: X \tau\left(A_{1}\right) \cdot d, y_{2}:=b\right]={ }_{\beta \eta} d .
\end{aligned}
$$

Since $Z$, the type of $v$, is atomic, $v$ is not an abstraction term. Since $Z$ is not $(\beta \eta$-convertible to) a sort, $v$ is not a product term. So $v$ is an atomic term, say $v \equiv \alpha M_{1} \ldots M_{n}$, for some $n \geq 0$. Here $\alpha$ is a sort or variable. Given that $v\left[y_{1}:=\lambda z: X \tau\left(A_{1}\right) \cdot c, y_{2}:=b\right]={ }_{\beta \eta} c$, we have that $\alpha \in\left\{y_{1}, y_{2}, c\right\}$. Given that $v\left[y_{1}:=\lambda z: X \tau\left(A_{1}\right) \cdot d, y_{2}:=b\right]={ }_{\beta \eta} d$, we have that $\alpha \in\left\{y_{1}, y_{2}, d\right\}$. So $\alpha \in\left\{y_{1}, y_{2}\right\}$. Towards a contradiction, suppose that $\alpha \equiv y_{2}$. Then $n=0$ and $v\left[y_{1}:=\lambda z: X \tau\left(A_{1}\right) . c, y_{2}:=b\right]={ }_{\beta \eta} b \neq \neq_{\beta \eta} c$. Contradiction. So $\alpha \equiv y_{1}$. Recall that $\Delta \vdash_{\lambda \underline{\omega}} y_{1}:\left(X \tau\left(A_{1}\right)\right) \rightarrow Z$. Hence $n=1$ and we have that

$$
\Delta \vdash_{\lambda \underline{\omega}} \alpha:\left(X \tau\left(A_{1}\right)\right) \rightarrow Z .
$$

Let us now take a look at $M_{1}$. Since $\tau$ is well-typed, the type of $M_{1}$ is $\beta \eta$-convertible to $X \tau\left(A_{1}\right)$. So the type of $M_{1}$ is atomic and as before we show that $M_{1}$ is an atomic term, say $M_{1} \equiv \beta N_{1} \ldots N_{k}$, for some $k \geq 0$. Here $\beta$ is a sort or a variable. For a contradiction, suppose that $\beta$ is a sort. Then $k=0$ and the type of $M_{1}$ would have to be $\beta \eta$-convertible to a sort, which is not the case. Contradiction. So $\beta$ is a variable. Since $\tau$ is closed, $\beta$ has to appear in a declaration $\beta: T$ in 
$\tau\left(\Gamma_{1}\right), \forall X: * \rightarrow *, \forall c: Z, \forall d: Z, \forall g: Z \rightarrow Z \rightarrow Z, \forall b: X \tau\left(B_{1}\right)$,

$\exists f:\left(\left(X \tau\left(A_{1}\right)\right) \rightarrow Z\right) \rightarrow\left(X \tau\left(B_{1}\right)\right) \rightarrow Z, \forall y_{1}:\left(X \tau\left(A_{1}\right)\right) \rightarrow Z, \forall y_{2}: X \tau\left(B_{1}\right)$.

The declaration $\beta: T$ can't appear in $\tau\left(\Gamma_{1}\right)$, because then the type of $M_{1}$ would be $*$, which is not $\beta \eta$-convertible to $X \tau\left(A_{1}\right)$. For the same reason, $\beta$ can't be $X$ or $Z$. Suppose $\beta \in\left\{c, d, g, f, y_{1}\right\}$. The types of these variables can all be written as $C_{1} \rightarrow \cdots \rightarrow C_{m} \rightarrow Z$, for some $m$ depending on the variable. So the type of $M_{1}$ would be $Z$, which is not $\beta \eta$-convertible to $X \tau\left(A_{1}\right)$. So $\beta \notin\left\{c, d, g, f, y_{1}\right\}$. Hence $\beta \equiv b$ or $\beta \equiv y_{2}$. Both $b$ and $y_{2}$ have type $X \tau\left(B_{1}\right)$. So we have $k=0$ and $M_{1} \equiv \beta$. Recall that the type of $M_{1}$ is $\beta \eta$-convertible to $X \tau\left(A_{1}\right)$. So we have that $X \tau\left(B_{1}\right)={ }_{\beta \eta} X \tau\left(A_{1}\right)$, which implies $\tau\left(A_{1}\right)={ }_{\beta \eta} \tau\left(B_{1}\right)$, as required.

Remark 6.5. Recall (see Definition 3.4) that we spoke about an alternative definition of closed solution, in which one only demands that substitution contexts are closed, whereas substitution terms need not be closed. The proof given above can be adapted to this definition. The step from $\left\langle\Gamma_{1} ; A_{1} ; B_{1}\right\rangle$ to $\left\langle\Gamma_{2} ; A_{2} ; B_{2}\right\rangle$ is in fact easier: one does not have to show that $\lambda x:\left(X \sigma\left(A_{1}\right)\right) \rightarrow Z . \lambda y: X \sigma\left(B_{1}\right) . x y$ is closed in $\tau\left(\Gamma_{2}\right)$. The step from $\left\langle\Gamma_{2} ; A_{2} ; B_{2}\right\rangle$ to $\left\langle\Gamma_{1} ; A_{1} ; B_{1}\right\rangle$ need not be changed.

\section{A Appendix}

In section 5, we have proved the decidability of third-order matching under a certain assumption, namely that universal variables of order $\infty$ do not occur. In this section we show that this assumption imposes no restriction. We present a translation that maps a matching problem $P$ of finite order involving universal variables of order $\infty$ to a matching problem $P^{\prime}$ without such variables. This translation has the property that $P$ has a solution iff $P^{\prime}$ has a solution. The crucial point here is that (writing $P=\langle\Gamma ; A ; B\rangle$ ), because $B$ is closed in $\Gamma$, variables of order $\infty$ do not occur in $B$. Also note that if $y$ is of order $\infty$ in $\Gamma$, then $\Gamma(y) \notin \mathcal{K}_{\square}$. Hence types do not contain variables of order $\infty$.

We introduce some terminology. Let $\Gamma$ be legal in $\lambda \underline{\omega}, y$ a variable of order $\infty$ in $\Gamma$. Write $\Gamma(y) \equiv Y_{1} \rightarrow \cdots \rightarrow Y_{k} \rightarrow Y$. If $Y$ is of finite order in $\Gamma$, then we call $y$ safe w.r.t. $\Gamma$. When $\Gamma$ is clear from the context, we simply say that $y$ is safe. Suppose $\Gamma \vdash_{\lambda \underline{\omega}} t: T$ and $\mathcal{L} \subseteq F V(t)$. Let $x \in \mathcal{L}$. If, for some occurrence of $x$ in $t$, there is no occurrence of a subterm of the form $y t_{1} \ldots t_{m}$ such that $y \in \mathcal{L}$ and this occurrence of $x$ is an occurrence in $t_{i}$, for some $1 \leq i \leq m$, then we call this occurrence of $x$ outermost w.r.t. $\mathcal{L}$ (in $t)$.

Lemma A.1. Suppose $\Gamma \vdash_{\lambda \underline{\omega}} t: T$, where $T$ is of finite order w.r.t. $\Gamma$. Write $\mathcal{L}=\{y \in$ $F V(t) \mid$ order of $y$ in $\Gamma$ is $\infty\}$. Let $y \in \mathcal{L}$. If $y$ is not safe, then for every occurrence of $y$ there is an occurrence of a subterm in $t$ of the form $z s_{1} \ldots s_{l}$ such that $z$ is safe, this occurrence of $z$ is outermost w.r.t. $\mathcal{L}$ and this occurrence of $y$ is an occurrence in $s_{i}$, for some $1 \leq i \leq l$.

Proof. By induction on the LNF-structure of $t$. Write $t \equiv \lambda x_{1}: T_{1} \ldots \lambda x_{n}: T_{n} \cdot x t_{1} \ldots t_{m}$. Let $y$ be given. If $x \equiv y$, then $y$ is safe. Otherwise $y \in F V\left(t_{1}\right) \cup \cdots \cup F V\left(t_{m}\right)$ (remember that $\left.y \notin \mathcal{K}_{\square}\right)$ and there are two possible cases. If $x$ is itself of order $\infty$, then we can take $x$ for $z$. If not, then $x$ is either an $x_{j}(1 \leq j \leq n)$ or a free variable of finite order. Say that 
the type of $x$ is $S_{1} \rightarrow \cdots \rightarrow S_{m} \rightarrow T^{\prime}$. In both cases each $S_{i}(1 \leq i \leq n)$ is of finite order in $\Gamma$. We also know that $\Gamma, \forall x_{1}: T_{1}, \ldots, \forall x_{n}: T_{n} \vdash_{\lambda \underline{\omega}} t_{i}: S_{i}$, for each $1 \leq i \leq n$. Write $J=\left\{1 \leq j \leq n \mid y \in F V\left(t_{j}\right)\right\}$. For all $j \in J$, we have by induction hypothesis: for every occurrence of $y$ in $t_{j}$ there is an occurrence of a subterm in $t_{j}$ of the form $z s_{1} \ldots s_{l}$ such that $z$ is safe w.r.t. $\Gamma, \forall x_{1}: T_{1}, \ldots, \forall x_{n}: T_{n}$ and this occurrence of $z$ is outermost w.r.t. $\mathcal{L} \cup\left\{u \in\left\{x_{1}, \ldots, x_{n}\right\} \mid \mathrm{u}\right.$ of order $\infty$ in $\left.\Gamma, \forall x_{1}: T_{1}, \ldots, \forall x_{n}: T_{n}\right\}$ in $t_{j}$ and this occurrence of $y$ is an occurrence in $s_{k}$, for some $1 \leq k \leq l$. Now note that none of the variables in $\left\{x_{1}, \ldots, x_{n}\right\}$ are of order $\infty$. So $z$ is not in this set and hence is safe w.r.t. $\Gamma$; the occurrence of $z$ is easily seen to be outermost w.r.t. $\mathcal{L}$ in $t$. This finishes the proof.

Definition A.2. Let $P=\langle\Gamma ; A ; B\rangle$ be a matching problem of finite order $n$ in $\lambda \underline{\omega}$. Suppose that $\Gamma \vdash_{\lambda \underline{\omega}} A: C$. We define $P^{\prime}=\left\langle\Gamma^{\prime} ; A^{\prime} ; B\right\rangle$ as follows. Write $\mathcal{L}=\{y \in F V(A) \mid$ order of $y$ in $\Gamma$ is $\infty\}$ and $\mathcal{L}^{\prime}=\{y \in \mathcal{L} \mid y$ safe in $\Gamma\}$. Note that very variable in $\mathcal{L}$ is universal in $\Gamma$. Let $\Gamma_{1}$ be the result of inserting immediately before each declaration $\forall y$ : $Y_{1} \rightarrow \cdots \rightarrow Y_{n} \rightarrow Y$ in $\Gamma$ with $y \in \mathcal{L}^{\prime}$ a declaration $\forall y^{\prime}: Y$, where $y^{\prime}$ is fresh. For each $y \in \mathcal{L}^{\prime}$ of type $Y_{1} \rightarrow \cdots \rightarrow Y_{n} \rightarrow Y$, write $c_{y} \equiv \lambda z_{1}: Y_{1} \ldots \lambda z_{1}: Y_{n} . y^{\prime}$, where $y^{\prime}$ is as above and $y^{\prime} \not \equiv z_{i}$, for all $1 \leq i \leq n$. Let $A_{1}$ be the result of replacing in $A$ each $y$ in $\mathcal{L}^{\prime}$ by $c_{y}$. By the Substitution Lemma and Weakening, $\Gamma_{1} \vdash_{\lambda \underline{\omega}} A_{1}: C$. So $\operatorname{lnf}_{\Gamma_{1}}\left(A_{1}\right)$ exists; we write $A^{\prime} \equiv \operatorname{lnf}_{\Gamma_{1}}\left(A_{1}\right)$. Using Lemma A.1, we know that no variable in $\mathcal{L}$ occurs in $A^{\prime}$ (this lemma is applicable because $C$ is closed in $\Gamma_{1}$, hence of finite order in $\Gamma_{1}$ ). Let $\Gamma^{\prime}$ be the result of removing all variables of order $\infty$ from $\Gamma_{1}$. Then by Thinning we have $\Gamma^{\prime} \vdash_{\lambda \underline{\omega}} A^{\prime}: C$ and $\Gamma^{\prime} \vdash_{\lambda \underline{\omega}} B: C$ (recall that $B$ and $C$ do not contain variables of order $\infty)$. So $\left\langle\Gamma^{\prime} ; A^{\prime} ; B\right\rangle$ is a matching problem of order $n$ and by construction $\Gamma^{\prime}$ and $A^{\prime}$ do not contain variables of order $\infty$.

Lemma A.3. Let $P=\langle\Gamma ; A ; B\rangle$ be a matching problem of finite order in $\lambda \underline{\omega}$. Let $P^{\prime}=$ $\left\langle\Gamma^{\prime} ; A^{\prime} ; B\right\rangle$ be as in Definition A.2. Then $P$ has a solution $\Leftrightarrow P^{\prime}$ has a solution.

Proof. Let $\mathcal{L}$ and $\mathcal{L}^{\prime}=\left\{y_{1}, \ldots, y_{l}\right\}$ and $c_{y}$ (for every $y \in \mathcal{L}^{\prime}$ ) be as in Definition A.2. Let $B^{\prime}$ be the normal form of $B$. Recall that no variable of order $\infty$ or a variable added in the construction of $\Gamma^{\prime}$ occurs in $B$ or $B^{\prime}$.

" $\Rightarrow$ " Suppose $P$ has solution $\sigma$. For each $y \in \mathcal{L}^{\prime}$ of type $Y_{1} \rightarrow \cdots \rightarrow Y_{n} \rightarrow Y$, write $c_{y}^{\prime} \equiv$ $\sigma\left(c_{y}\right) \equiv \sigma_{\square}\left(c_{y}\right) \equiv \lambda z_{1}: \sigma\left(Y_{1}\right) \ldots \lambda z_{1}: \sigma\left(Y_{n}\right) . y^{\prime}$. Write $\sigma_{\square}=\left\{\left\langle z_{j} ; \gamma_{j} ; Z_{j}\right\rangle \mid 1 \leq j \leq m_{1}\right\}$ and $\sigma_{*}=\left\{\left\langle x_{j} ; \delta_{j} ; S_{j}\right\rangle \mid 1 \leq j \leq m_{2}\right\}$. For each $1 \leq j \leq m_{2}$, let $S_{j}^{\prime}$ be the result of replacing each $y \in F V\left(S_{j}\right) \cap \mathcal{L}^{\prime}$ by $c_{y}^{\prime}$ and let $S_{j}^{\prime \prime}$ be $\operatorname{lnf}_{\sigma\left(\Gamma_{1}\right)}\left(S_{j}^{\prime}\right)$. (One can check that this is well-defined.) Put $\sigma^{\prime}=\sigma_{\square} \cup\left\{\left\langle x_{j} ; \delta_{j} ; S_{j}^{\prime \prime}\right\rangle \mid 1 \leq j \leq m_{2}\right\}$. We show that $\sigma^{\prime}$ is a solution for $P^{\prime}$. Using Lemma A.1, $\sigma^{\prime}$ is easily seen to be well-typed in $\Gamma^{\prime}$. Below we calculate that $A\left[\vec{y}:=\overrightarrow{c_{y}}\right][\vec{z}:=\vec{Z}]\left[\vec{x}:=\overrightarrow{S^{\prime \prime}}\right]={ }_{\beta \eta} B^{\prime}$. Since $A\left[\vec{y}:=\overrightarrow{c_{y}}\right]={ }_{\beta \eta} A^{\prime}$, we have that $A^{\prime}[\vec{z}:=\vec{Z}]\left[\vec{x}:=\overrightarrow{S^{\prime \prime}}\right]=\beta \eta B^{\prime}$. We may conclude that $\sigma^{\prime}$ is a solution for $P^{\prime}$. As to the calculation. We know $A[\vec{z}:=\vec{Z}][\vec{x}:=\vec{S}] \rightarrow \beta \eta B^{\prime}$. Now

$$
\begin{aligned}
& A\left[\vec{y}:=\overrightarrow{c_{y}}\right][\vec{z}:=\vec{Z}]\left[\vec{x}:=\overrightarrow{S^{\prime \prime}}\right] \equiv \\
& A[\vec{z}:=\vec{Z}]\left[\vec{y}:=\overrightarrow{c_{y}^{\prime}}\right]\left[\vec{x}:=\overrightarrow{S^{\prime \prime}}\right]=\beta \eta \\
& A[\vec{z}:=\vec{Z}]\left[\vec{y}:=\overrightarrow{c_{y}^{\prime}}\right]\left[\vec{x}:=\overrightarrow{S^{\prime}}\right] \equiv \\
& A[\vec{z}:=\vec{Z}][\vec{x}:=\vec{S}]\left[\vec{y}:=\overrightarrow{c_{y}^{\prime}}\right] \rightarrow{ }^{\prime}{ }^{\prime} \\
& B^{\prime}\left[\vec{y}:=\overrightarrow{c_{y}^{\prime}}\right] \equiv B^{\prime} .
\end{aligned}
$$


We have to verify the validity of the steps in the calculation. Note that all terms involved are typable in $\sigma\left(\Gamma_{1}\right)$. As to the first step. Observe that $\left\{z_{1}, \ldots, z_{m_{1}}\right\} \cap\left\{y_{1}, \ldots, y_{l}\right\}=\emptyset$, because the variables in the leftmost set are existentially quantified in $\Gamma_{1}$, whereas the variables in the rightmost set (i.e. $\mathcal{L}^{\prime}$ ) are universally quantified in $\Gamma_{1}$. Moreover the variables in $\mathcal{L}^{\prime}$ are of order $\infty$, hence do not occur in $\sigma_{\square}$. The second step is easy. As to the third step. Again we have that $\left\{x_{1}, \ldots, x_{m_{2}}\right\} \cap\left\{y_{1}, \ldots, y_{l}\right\}=\emptyset$. Moreover no variable in $\left\{x_{1}, \ldots, x_{m_{2}}\right\}$ occurs in a term $c_{y}^{\prime}$. The fourth step follows from Substitutivity and the fact that $A[\vec{z}:=\vec{Z}][\vec{x}:=\vec{S}] \rightarrow_{\beta \eta} B^{\prime}$.

" $\Leftarrow$ " Suppose $P^{\prime}$ has solution $\tau$. By Proposition 5.29, we may assume that the substitution terms in $\tau$ do not contain any of the new variables $y^{\prime}$ or any variable of order $\infty$. In particular, $\tau$ is well-typed in $\Gamma$. For each $y \in \mathcal{L}^{\prime}$ of type $Y_{1} \rightarrow \cdots \rightarrow Y_{n} \rightarrow Y$, write $c_{y}^{\prime \prime} \equiv \tau\left(c_{y}\right) \equiv \lambda z_{1}: \tau\left(Y_{1}\right) \ldots \lambda z_{1}: \tau\left(Y_{n}\right) \cdot y^{\prime}$. Let $A^{\prime \prime}$ be the normal form of $\tau(A)$ ( $A^{\prime \prime}$ exists because $\Gamma \vdash_{\lambda \underline{\omega}} A: C$ and $\tau$ is well-typed in $\Gamma$ ). We prove that $A^{\prime \prime} \equiv B^{\prime}$. First, we claim that $A^{\prime \prime}$ does not contain variables from the set $\mathcal{L}$. Towards a contradiction, assume that $A^{\prime \prime}$ does contain such variables. Then it contains an outermost occurrence of such a variable $u$. There are two possible cases: the first case is where $u$ is safe in $\Gamma$, the second case is where $u$ is not safe in $\Gamma$. Let $A^{\prime \prime \prime}$ be the result of replacing in $A^{\prime \prime}$ each $y$ in $\mathcal{L}^{\prime}$ by $c_{y}^{\prime \prime}$ (this term is well-typed in $\tau\left(\Gamma_{1}\right)$ ). In the first case, the normal form of $A^{\prime \prime \prime}$ contains an occurrence of $u^{\prime}$. In the second case the normal form of $A^{\prime \prime \prime}$ contains an occurrence of $u$. But the normal form of $A^{\prime \prime \prime}$ is $B^{\prime}$. This can be seen as follows. Write $\tau_{\square}=\left\{\left\langle z_{j} ; \gamma_{j} ; Z_{j}\right\rangle \mid 1 \leq j \leq m_{1}\right\}$ and $\tau_{*}=\left\{\left\langle x_{j} ; \delta_{j} ; S_{j}\right\rangle \mid 1 \leq j \leq m_{2}\right\}$. We know $A[\vec{z}:=\vec{Z}][\vec{x}:=\vec{S}] \rightarrow \beta \eta A^{\prime \prime}$. So $A[\vec{z}:=\vec{Z}][\vec{x}:=\vec{S}]\left[\vec{y}:=\overrightarrow{c_{y}^{\prime \prime}}\right] \rightarrow \beta \eta A^{\prime \prime}\left[\vec{y}:=\overrightarrow{c_{y}^{\prime \prime}}\right] \equiv A^{\prime \prime \prime}$. Now

$A[\vec{z}:=\vec{Z}][\vec{x}:=\vec{S}]\left[\vec{y}:=\overrightarrow{c_{y}^{\prime \prime}}\right] \equiv \quad\left(\right.$ no $y \in \mathcal{L}^{\prime}$ free in $\left.\vec{S}\right)$

$A[\vec{z}:=\vec{Z}]\left[\vec{y}:=\overrightarrow{c_{y}^{\prime \prime}}\right][\vec{x}:=\vec{S}] \equiv$

$A\left[\vec{y}:=\overrightarrow{c_{y}}\right][\vec{z}:=\vec{Z}][\vec{x}:=\vec{S}]=\beta \eta$

$A^{\prime}[\vec{z}:=\vec{Z}][\vec{x}:=\vec{S}]$.

Recall that $B^{\prime}$ is the normal form of $A^{\prime}[\vec{z}:=\vec{Z}][\vec{x}:=\vec{S}]$. Thus we have that $B^{\prime}$ is also the normal form of $A[\vec{z}:=\vec{Z}][\vec{x}:=\vec{S}]\left[\vec{y}:=\overrightarrow{c_{y}^{\prime \prime}}\right]$ and hence of $A^{\prime \prime \prime}$. And $B^{\prime}$ does not contain $u$ or $u^{\prime}$. Contradiction. We conclude that $A^{\prime \prime}$ does not contain variables from the set $\mathcal{L}$. So $A^{\prime \prime}\left[\vec{y}:=\overrightarrow{c_{y}^{\prime \prime}}\right] \equiv A^{\prime \prime}$. From this we quickly obtain that $A^{\prime \prime} \equiv B^{\prime}$, because $B^{\prime}$ is the normal form of $A^{\prime \prime}\left[\vec{y}:=\overrightarrow{c_{y}^{\prime \prime}}\right] \equiv A^{\prime \prime}$ and $A^{\prime \prime}$ is itself normal.

Remark A.4. With a slightly more complicated proof, also removing variables of order $\infty$ from substitution terms, one obtains the following strengthening. Let $P$ and $P^{\prime}$ be as in Lemma A.3. Then $P$ has a solution $\Leftrightarrow P^{\prime}$ has a solution not containing variables of order $\infty$.

\section{References}

[1] H.P. Barendregt. The Lambda Calculus. Its Syntax and Semantics. North-Holland, Amsterdam, second, revised edition, 1984.

[2] H.P. Barendregt. Lambda calculi with types. In S. Abramsky, D. M. Gabbay, and T.S.E. Maibaum, editors, Handbook of Logic in Computer Science, volume 2, pages 117-309. Oxford Science Publications, 1992.

[3] T. Coquand and G. Huet. The calculus of constructions. Information and Control, 76:95-120, 1988. 
[4] G. Dowek. Démonstration Automatique dans le Calcul des Constructions. PhD thesis, l'université Paris VII, 1991.

[5] G. Dowek. L'indécidabilité du filtrage du troisième ordre dans les calculs avec types dépendants ou constructeurs de types. Compte Rendu à l'Académie des Sciences, 312, Série I:951-956, 1991.

[6] G. Dowek. A second-order pattern matching algorithm for the cube of typed $\lambda$-calculi. In Mathematical Foundations of Computer Science 91, volume 520 of Lecture Notes in Computer Science, pages 151-160. Springer-Verlag, 1991.

[7] G. Dowek. A second-order pattern matching algorithm for the cube of typed $\lambda$-calculi. Technical report, INRIA - Rocquencourt, 1991.

[8] G. Dowek. Third order matching is decidable. In Proceedings $7^{\text {th }}$ Annual Symposium on Logic in Computer Science, Santa Cruz, California, 1992.

[9] G. Dowek. A complete proof synthesis method for the cube of type systems. To be published, 1993.

[10] G. Dowek. The undecidability of pattern matching in calculi where primitive recursive functions are representable. Theoretical Computer Science, 107:349-356, 1993.

[11] G. Dowek, A. Felty, H. Herbelin, G. Huet, C. Murthy, C. Parent, C. Paulin-Mohring, and B. Werner. The Coq proof assistant user's guide. Version 5.8. Technical report, INRIA - Rocquencourt, May 1993.

[12] J.H. Geuvers. The Church-Rosser property for $\beta \eta$-reduction in typed $\lambda$-calculi. In Proceedings $7^{\text {th }}$ Annual Symposium on Logic in Computer Science, Santa Cruz, California, 1992.

[13] J.H. Geuvers and M.-J. Nederhof. Modular proof of strong normalisation for the calculus of constructions. Journal of Functional Programming, 1:155-189, 1989.

[14] W.D. Goldfarb. The undecidability of the second-order unification problem. Theoretical Computer Science, 13:225-230, 1981.

[15] Z. Luo. ECC, the Extended Calculus of Constructions. In Proceedings $4^{\text {th }}$ Annual Symposium on Logic in Computer Science, Asilomar, California, pages 386-395. IEEE, 1989.

[16] Z. Luo and R. Pollack. LEGO proof development system: User's manual. Technical report, Department of Computer Science, University of Edinburgh, May 1992.

[17] D. Miller. Unification under a mixed prefix. Journal of Symbolic Computation, 14(4):321-359, October 1992.

[18] V. Padovani. Fourth order dual interpolation is decidable. Manuscript, Université Paris VII C.N.R.S, 1994.

[19] V. Padovani. On equivalence classes of interpolation equations. Manuscript, Université Paris VII - C.N.R.S, 1994. 